\title{
Lagrange's four squares theorem with one prime and three almost-prime variables
}

\author{
D. R. Heath-Brown D. I. Tolev
}

\section{Introduction and statement of the results.}

In this paper we study the equation of Lagrange

$$
x_{1}^{2}+x_{2}^{2}+x_{3}^{2}+x_{4}^{2}=N
$$

with multiplicative restrictions imposed on the variables.

Various proofs for the solvability of (1) in integers $x_{1}, x_{2}, x_{3}, x_{4}$ are known. We should mention the explicit formula for the number of integer solutions of (1), discovered by Jacobi. We refer the reader to the books of Hardy and Wright [7] and Hua [11], for example.

Kloosterman [16] considered the problem of the representation of large integers $N$ by the integral positive definite quadratic form

$$
a_{1} x_{1}^{2}+a_{2} x_{2}^{2}+a_{3} x_{3}^{2}+a_{4} x_{4}^{2} .
$$

His method yields an asymptotic formula for the number of representations. The classical circle method of Hardy, Littlewood and Ramanujan provides an asymptotic formula for quadratic forms with five or more variables only.

It is expected that every sufficiently large integer $N$, satisfying the congruence condition $N \equiv 4(\bmod 24)$, can be represented in the form $(1)$ with prime variables $x_{i}$. This hypothesis has not been proved so far. Using Vinogradov's method for the solution of the ternary Goldbach problem, however, Hua [10] proved that all large integers, satisfying a natural congruence condition, are sums of five squares of primes.

Greaves [6], Plaksin [17] and Shields [18] proved the solvability of (1) with two prime and two integer variables. Actually, in [17] and [18] an asymptotic formula for the number of solutions was found.

Brüdern and Fouvry [1] considered (1) with sufficiently large $N$ satisfying $N \equiv 4(\bmod 24)$ and found a lower bound for the number of solutions in integers $x_{i}$ with no more than 34 prime factors each.

The main purpose of the present paper is to prove the following: 
Theorem 1. Every sufficiently large integer $N$, satisfying $N \equiv 4(\bmod 24)$, can be represented in the form

$$
p^{2}+x_{1}^{2}+x_{2}^{2}+x_{3}^{2}=N,
$$

where $p$ is a prime and $x_{i}$ are integers without prime factors less than $N^{0.004915}$. The number of such representations exceeds $c N(\log N)^{-4}$ for some positive constant $c$. In particular, every such $x_{i}$ has at most 101 prime factors.

We are also in a position to improve slightly the result of Brüdern and Fouvry from [1]. We have

Theorem 2. Every sufficiently large integer $N$, satisfying $N \equiv 4(\bmod 24)$, can be represented in the form (1), where $x_{i}$ are integers without prime factors less than $N^{0.01995}$. The number of such representations exceeds $c N(\log N)^{-4}$ for some positive constant $c$. In particular, every such $x_{i}$ has at most 25 prime factors.

In spite of the results of Greaves, Plaksin and Shields already referred to, there seems little hope at present of establishing a version of Lagrange's Theorem involving two primes and two almost-primes. Although we can show that $N-p^{2}-q^{2}$ is a sum of two squares, we are unable to control sufficiently the divisibility properties of the two squares that arise. To prove Theorem 1 we have to show that $N-p^{2}$ is a sum of three squares, and to control the distribution in residue classes of these three squares. A standard application of the circle method, with the Kloosterman refinement, is not quite sufficient for this purpose. However by using the first author's "square sieve" [8], we are able to take advantage of the fact that we are considering numbers $N-p^{2}$ in which $p^{2}$ is a square, and this leads to a suitable saving. The usual machinery of the Kloosterman refinement is needed, but it appears that, at one point, when we consider the second estimate for $E\left(G_{1}, G_{2}\right)$ in $\S 3.4 .6$, this is insufficient. This rather technical problem is overcome by using a double Kloosterman refinement. Thus, if the Farey dissection in our application of the circle method involves arcs around the points $a / q$, we average non-trivially not only over the numerators $a$, but also over the denominators $q$. It would seem that this is the first occasion on which such a technique has been successfully employed to give an unconditional result.

Acknowledgements. The main part of this research was done during the visit of the second author to the Mathematical Institute of the University of Oxford. The second author thanks the Royal Society for financial support, the staff of the Institute for the excellent working conditions and also Plovdiv University Scientific Fund (grant PU2-MM) for covering some other expenses.

\section{$2 \quad$ Notations and some Lemmas.}

Denote $P=N^{1 / 2}$. Let $\varepsilon \in\left(0,10^{-6}\right)$ be an arbitrarily small fixed number. Let $A>10^{6}$ be an arbitrarily large number, which may not be the same in 
different occurrences. If it is not stated explicitly, the constants in $\mathcal{O}$-terms and $\ll-$ symbols are absolute or depend on $\varepsilon$ and $A$. The letter $p$ is reserved for prime numbers. In all sections, with the exception of Section 5, we denote by bold style letters three-dimensional vectors. In Section 5 we use bold style letters to denote four-dimensional vectors. We denote by $\left(m_{1}, \cdots, m_{k}\right)$ and $\left[m_{1}, \cdots, m_{k}\right]$ the greatest common divisor and, respectively, the least common multiple of the integers of $m_{1}, \cdots, m_{k}$. However, if $u, v$ are real numbers then $(u, v)$ means the interval with endpoints $u$ and $v$. The meaning is always clear from the context.

As usual, $\mu(n), \varphi(n), \tau(n), \nu(n)$ denote the Möbius function, Euler's function, the number of divisors of $n$ and the number of distinct prime factors of $n$, respectively. Instead of $m \equiv n(\bmod k)$ we write for simplicity $m \equiv n(k)$. If $p^{l} \mid m$, but $p^{l+1} \nmid m$ then we write $p^{l} \| m$. We denote $e(\alpha)=e^{2 \pi i \alpha}$ and $e_{q}(\alpha)=e(\alpha / q)$. By $[t]$ we denote the integer part of the real number $t$ and let $\|t\|$ be the distance from $t$ to the nearest integer. We use $\sum_{x(q)}$ and $\sum_{x(q) *}$ to denote sums with $x$ running over a complete system, respectively reduced system of residues modulo $q$. If $q$ is an odd integer then by $\left(\frac{l}{q}\right)$ we denote the Jacobi symbol. If $(a, q)=1$ then $\overline{(a)}_{q}$ means the inverse of $a$ modulo $q$. If the value of the modulus is clear from the context then we simply write $\bar{a}$. For example, $e_{q}(\bar{a})$ always means $e_{q}\left(\overline{(a)}_{q}\right)$. For any $a$ we put $e_{1}(\bar{a})=1$. If $U$ and $V$ are positive then $U \asymp V$ means that $U \ll V \ll U$. We use $\square$ to mark an end of a proof or its absence.

We denote by $\pi(x, q, m)$ the number of primes $p \leq x$ such that $p \equiv m(q)$ and let

$$
\Delta(x, q, m)=\pi(x, q, m)-\frac{1}{\varphi(q)} \int_{2}^{x} \frac{d t}{\log t} .
$$

Our first lemma is a weak version of the Barban-Davenport-Halberstam theorem (see Chapter 29 of [3], for example).

Lemma 1. Suppose that $Q \ll x^{1-\varepsilon}$. Then we have

$$
\sum_{q \leq Q} \sum_{m(q)^{*}} \Delta(x, q, m)^{2} \ll x^{2}(\log x)^{-A} .
$$

In our study we use the properties of the Kloosterman sum $K(q, m, n)$, Ramanujan sum $c_{q}(n)$ and the Gauss sums $\gamma(q), S(q, a, m), S(q, a)$ and $T(q, a)$. 
These sums are defined by

$$
\begin{aligned}
K(q, m, n) & =\sum_{x(q)^{*}} e_{q}(m x+n \bar{x}), \quad c_{q}(m)=K(q, m, 0), \\
\gamma(q) & =\sum_{l(q)}\left(\frac{l}{q}\right) e_{q}(l) \quad(\text { for odd integers } q \text { only }), \\
S(q, m, n) & =\sum_{x(q)} e_{q}\left(m x^{2}+n x\right), \quad S(q, m)=S(q, m, 0), \\
T(q, m) & =\sum_{x(q)^{*}} e_{q}\left(m x^{2}\right) .
\end{aligned}
$$

If $\mathbf{d} \in \mathbb{N}^{3}$ and $\mathbf{n} \in \mathbb{Z}^{3}$ then we denote

(8) $\quad S_{\mathbf{d}}(q, m, \mathbf{n})=\prod_{i=1}^{3} S\left(q, m d_{i}^{2}, n_{i}\right), \quad S_{\mathbf{d}}(q, m)=S_{\mathbf{d}}(q, m, \mathbf{0})$.

In Section 5, however, bold style vectors denote four-dimensional vectors and the definition of $S_{\mathbf{d}}(q, m, \mathbf{n})$ is different.

We also put

$$
h_{\mathbf{d}}(q)=q^{-3} \varphi(q)^{-1} \sum_{a(q)^{*}} S_{\mathbf{d}}(q, a) T(q, a) e_{q}(-a N) .
$$

In the next lemma we present some identities and inequalities for the sum $S(q, m, n)$. The proof of $(i)$ is elementary. The proofs of $(i i)-(v i)$ are available in Section 6 of [4] and Chapter 7 of [11].

Lemma 2. The Gauss sum $S(q, m, n)$ satisfies:

(i) If $\left(q_{1}, q_{2}\right)=1$ then

$$
S\left(q_{1} q_{2}, a_{1} q_{2}+a_{2} q_{1}, n\right)=S\left(q_{1}, a_{1} q_{2}^{2}, n\right) S\left(q_{2}, a_{2} q_{1}^{2}, n\right) .
$$

(ii) Suppose that $(q, m)=d$. We have

$$
S(q, m, n)= \begin{cases}d S(q / d, m / d, n / d) & \text { if } d \mid n, \\ 0 & \text { if } d \nmid n .\end{cases}
$$

(iii) If $(q, m)=1$ then $|S(q, m, n)| \leq 2 q^{1 / 2}$.

(iv) If $(q, 2 m)=1$ then

$$
S(q, m, n)=e_{q}\left(-\overline{(4 m)} n^{2}\right)\left(\frac{m}{q}\right) S(q, 1) .
$$

(v) If $(q, 2)=1$ then

$$
S(q, 1)=\left\{\begin{array}{lll}
q^{1 / 2} & \text { if } & q \equiv 1(4), \\
i q^{1 / 2} & \text { if } & q \equiv-1(4) .
\end{array}\right.
$$


(vi) If $(a, 2)=1$ then

$$
S\left(2^{l}, a\right)= \begin{cases}0 & \text { if } l=1 \\ 2^{l / 2}\left(1+i^{a}\right) & \text { if } l \text { is even } \\ 2^{(l+1) / 2} e(a / 8) & \text { if } l>1 \text { and odd } .\end{cases}
$$

In the next lemma we study $T(q, m)$. Again the proof of $(i)$ is elementary. Identities (ii) and (iii) can be easily verified using definition (7) and Lemma 2.

Lemma 3. The Gauss sum $T(q, m)$ satisfies:

(i) If $\left(q_{1}, q_{2}\right)=1$ then

$$
T\left(q_{1} q_{2}, a_{1} q_{2}+a_{2} q_{1}\right)=T\left(q_{1}, a_{1}\right) T\left(q_{2}, a_{2}\right) .
$$

(ii) If $p>2$ is a prime and $(p, a)=1$ then

$$
T\left(p^{l}, a\right)= \begin{cases}S(p, a)-1 & \text { if } l=1, \\ 0 & \text { if } l>1 .\end{cases}
$$

(iii) If $(a, 2)=1$ then

$$
T\left(2^{l}, a\right)= \begin{cases}-1 & \text { if } l=1 \\ 2 e(a / 4) & \text { if } l=2, \\ 4 e(a / 8) & \text { if } l=3 \\ 0 & \text { if } l>3\end{cases}
$$

In the next lemma we present Weil's estimate for the Kloosterman sum. The most impostant case, when $q$ is a prime, was considered by Weil [21]. For the proof in the general case we refer the reader to Estermann [5].

Lemma 4. We have

$$
|K(q, m, n)| \leq \tau(q) q^{1 / 2}(m, n, q)^{1 / 2} .
$$

A simple identity and an estimate for the Ramanujan sum are given below. A proof is available in Chapter 16 of [7].

Lemma 5. We have

$$
c_{q}(n)=\frac{\varphi(q)}{\varphi\left(\frac{q}{(q, n)}\right)} \mu\left(\frac{q}{(q, n)}\right) .
$$

In particular

$$
\left|c_{q}(n)\right| \leq(q, n) .
$$


In the next lemma we give some properties of the Gauss sum $\gamma(q)$. Proofs can be found in Chapter 7 of [11].

\section{Lemma 6.}

(i) For any odd integer $q$ we have $|\gamma(q)| \leq q^{1 / 2}$.

(ii) If $p>2$ is a prime then $\gamma(p)=S(p, 1)$.

In the next lemma we present the fundamental properties of the Jacobi symbol. A proof is available, for example, in Chapter 3 of [11].

Lemma 7. If $q$ and $q_{1}$ are odd integers and $\left(q, q_{1}\right)=1$ then we have

$$
\begin{aligned}
\text { (i) } & \left(\frac{q}{q_{1}}\right)\left(\frac{q_{1}}{q}\right)=(-1)^{\frac{q-1}{2} \cdot \frac{q_{1}-1}{2}}, \\
\text { (ii) } & \left(\frac{2}{q}\right)=(-1)^{\frac{q^{2}-1}{8}}, \\
\text { (iii) } & \left(\frac{-1}{q}\right)=(-1)^{\frac{q-1}{2}} .
\end{aligned}
$$

Now we shall study the function $h_{\mathbf{d}}(q)$, defined by (9). For any prime $p>2$ we put

$$
\begin{aligned}
& h_{0}(p)= \begin{cases}\frac{1}{p} & \text { if } p \mid N, \\
\frac{-1}{p(p-1)}\left(1+\left(\frac{-N}{p}\right)\right) & \text { if } p \nmid N ;\end{cases} \\
& h_{1}(p)= \begin{cases}\frac{-1}{p}\left(\frac{-1}{p}\right) & \text { if } p \mid N, \\
\frac{1}{p-1}\left(\left(\frac{-N}{p}\right)+\frac{1}{p}\left(\frac{-1}{p}\right)\right) & \text { if } p \nmid N ;\end{cases} \\
& h_{2}(p)= \begin{cases}\left(\frac{-1}{p}\right) & \text { if } p \mid N, \\
\frac{-1}{p-1}\left(\left(\frac{-1}{p}\right)+\left(\frac{N}{p}\right)\right) & \text { if } p \nmid N ;\end{cases} \\
& h_{3}(p)= \begin{cases}-1 & \text { if } p \mid N, \\
\frac{1}{p-1}\left(p\left(\frac{N}{p}\right)+1\right) & \text { if } p \nmid N .\end{cases}
\end{aligned}
$$

The following lemma holds: 
Lemma 8. Suppose that $d_{1}, d_{2}, d_{3}$ are squarefree odd integers and $N \equiv 4(24)$. The function $h_{\mathbf{d}}(q)$ is multiplicative with respect to $q$. We have

$$
h_{\mathbf{d}}\left(2^{l}\right)= \begin{cases}0 & \text { if } l=1, \\ -1 / 2 & \text { if } l=2, \\ 1 / 2 & \text { if } l=3, \\ 0 & \text { if } l>3 .\end{cases}
$$

If $p>2$ is a prime then

$$
h_{\mathbf{d}}\left(p^{l}\right)= \begin{cases}h_{j}(p) & \text { if } p^{j} \| d_{1} d_{2} d_{3} \quad \text { and } l=1, \\ 0 & \text { if } l>1 .\end{cases}
$$

The series $\Sigma_{0}$, defined below, is absolutely convergent and

$$
\Sigma_{0}=\Sigma_{0}(\mathbf{d}, N)=\sum_{q=1}^{\infty} h_{\mathbf{d}}(q)=\prod_{p>2}\left(1+h_{\mathbf{d}}(p)\right) .
$$

Proof: The multiplicativity of $h_{\mathbf{d}}(q)$ with respect to $q$ follows easily from Lemmas $2(i)$ and $3(i)$. Formulas (14) and (15) are consequences of Lemma 2 $(v),(v i)$ and Lemma $3(i i)$, (iii). From (10) - (15) we find that the following estimate holds:

$$
h_{\mathbf{d}}(q) \ll \mu^{2}\left(\frac{q}{(q, 4)}\right) \frac{6^{\nu(q)}}{q^{2}}(q, N)\left(d_{1} d_{2} d_{3}\right)^{3} .
$$

Hence the series $\Sigma_{0}$ is absolutely convergent and applying Euler's identity we get (16).

Consider the function

$$
\omega_{0}(t)= \begin{cases}\exp \left(\frac{1}{(20 t-10)^{2}-1}\right) & \text { if } t \in\left(\frac{9}{20}, \frac{11}{20}\right), \\ 0 & \text { otherwise }\end{cases}
$$

It is infinitely differentiable on the real line. Denote

$$
\omega(x)=\omega_{0}\left(x P^{-1}\right), \quad \omega(\mathbf{x})=\omega\left(x_{1}\right) \omega\left(x_{2}\right) \omega\left(x_{3}\right)
$$

and

$$
I(\beta, u)=\int_{-\infty}^{\infty} \omega_{0}(x) e\left(\beta x^{2}+u x\right) d x, \quad I(\beta)=I(\beta, 0) .
$$

If $\mathbf{d} \in \mathbb{N}^{3}$ and $\mathbf{u} \in \mathbb{R}^{3}$ then we define

$$
I_{\mathbf{d}}(\beta, \mathbf{u})=\prod_{i=1}^{3} I\left(\beta, u_{i} d_{i}^{-1}\right) .
$$

In Section 5 , however, the definition of $I_{\mathbf{d}}(\beta, \mathbf{u})$ is different. 
Lemma 9. The following estimates hold:

$$
\begin{aligned}
& I(\beta, u) \ll\left(1+|\beta|^{k}\right)|u|^{-k} \quad \text { for } u \neq 0 \text { and for any } k \in \mathbb{N}, \\
& \text { (ii) } \quad I(\beta) \ll \min \left(1,|\beta|^{-k}\right) \quad \text { for any } k \in \mathbb{N} \text {, } \\
& \text { (iii) } \quad I(\beta, u) \ll \min \left(1,|\beta|^{-1 / 2}\right) \text {. }
\end{aligned}
$$

The constants in the $\ll-$ symbols in $(i)$ and (ii) depend on $k$.

Proof: We prove $(i)$ by multiple partial integration. To prove (ii) we change the variable $x^{2}=y$ and then proceed as in the proof of $(i)$. Finally, the estimate (iii) is well-known.

\section{Lemma 10.}

(i) Suppose that $u=\max \left(\left|u_{1}\right|, \ldots,\left|u_{6}\right|\right)>0$. Then we have

$$
J=\int_{-\infty}^{\infty}\left|I\left(\beta, u_{1}\right) \ldots I\left(\beta, u_{6}\right)\right| d \beta \ll u^{-2+\varepsilon} .
$$

(ii) Suppose that $v=\max \left(\left|v_{1}\right|, \ldots,\left|v_{4}\right|\right)>0$. Then we have

$$
\int_{-\infty}^{\infty}\left|I\left(\beta, v_{1}\right) \ldots I\left(\beta, v_{4}\right)\right| d \beta \ll v^{-1+\varepsilon}
$$

Proof: We prove only $(i)$. The proof of $(i i)$ is similar. If $u \leq 1$ the last inequality is a consequence of the trivial estimate $J \ll 1$. Suppose that $u>1$. We have $J=J_{1}+J_{2}$, where in $J_{1}$ we integrate over $|\beta| \leq u^{1-\varepsilon / 2}$ and in $J_{2}$ over the other $\beta$. Lemma 9 (iii) implies that $J_{2} \ll \int_{u^{1-\varepsilon / 2}}^{\infty} \beta^{-3} d \beta \ll u^{-2+\varepsilon}$.

Now consider $J_{1}$. Suppose that $u=\left|u_{1}\right|$. We take the integer $k=\left[6 \varepsilon^{-1}\right]$. If $|\beta| \leq\left|u_{1}\right|^{1-\varepsilon / 2}$ then Lemma $9(i)$ gives $I\left(\beta, u_{1}\right) \ll\left(1+|\beta|^{k}\right)\left|u_{1}\right|^{-k} \ll$ $\left|u_{1}\right|^{(1-\varepsilon / 2) k-k}$. Therefore $J_{1} \ll\left|u_{1}\right|^{(1-\varepsilon / 2)(k+1)-k} \ll\left|u_{1}\right|^{-2}$ and the result follows.

Using Lemma 9 we see that the Fourier thansform of $I^{3}(\beta)$

$$
H(t)=\int_{-\infty}^{\infty} I^{3}(\beta) e(-t \beta) d \beta
$$

is uniformly and absolutely convergent. We have

Lemma 11. The function $H(t)$ is non-negative and infinitely many times differentiable. It is supported and positive in $\left(t_{1}, t_{2}\right)$ for some $t_{1}, t_{2}$ such that $0<t_{1}<t_{2}<1$. We have

$$
I^{3}(\beta)=\int_{-\infty}^{\infty} H(t) e(\beta t) d t
$$


and

$$
\kappa_{0}=\int_{-\infty}^{\infty}|I(\beta)|^{6} d \beta=\int_{-\infty}^{\infty} H^{2}(t) d t .
$$

The integral

$$
\kappa_{1}=\int_{-\infty}^{\infty} I^{4}(\beta) e(-\beta) d \beta
$$

is absolutely convergent and the constant $\kappa_{1}$, defined above, is real and positive.

We also have

$$
\int_{-\infty}^{\infty} H^{2}(t) e(\beta t) d t \ll \min \left(1,|\beta|^{-k}\right)
$$

for any integer $k$. The constant in the $\ll-$ symbol depends on $k$.

Proof: The smoothness of $H(t)$ as well as the identities (22) and (23) are well known facts from Fourier analysis. To prove the estimate (25) we use multiple partial integration. Finally, we can prove the other statements by the standard technique of the circle method (see Chapter 11 of [15], for example).

We can now define

$$
\mathcal{N}_{0}=\mathcal{N}_{0}(N)=P \int_{t_{0} P}^{P} H\left(1-\frac{x^{2}}{P^{2}}\right) \frac{d x}{\log x},
$$

where $t_{0}=\left(1-t_{2}\right)^{1 / 2} \in(0,1)$ and $t_{2}$ is specified in Lemma 11 . Obviously

$$
\mathcal{N}_{0} \asymp \frac{P^{2}}{\log P} .
$$

\section{$3 \quad$ Propositions which imply Theorem 1.}

\subsection{Statement of the propositions.}

Define

$$
\Omega_{\mathbf{d}}(n)=\sum_{\substack{x_{1}^{2}+x_{2}^{2}+x_{3}^{2}=n \\ x_{i} \equiv 0\left(d_{i}\right)}} \omega(\mathbf{x}) .
$$

A formal application of the circle method suggests that the sum $\Omega_{\mathbf{d}}(n)$ can be approximated (at least in some average sense) by the expression

$$
\mathcal{M}_{\mathbf{d}, Q}(n)=\frac{P H\left(n N^{-1}\right)}{d_{1} d_{2} d_{3}} \sum_{q \leq Q} q^{-3} \sum_{a(q)^{*}} e_{q}(-a n) S_{\mathbf{d}}(q, a) .
$$


It is clear that $\mathcal{M}_{\mathbf{d}, Q}(n)$ is always real. Denote

$$
\mathcal{G}_{\mathbf{d}, Q}(n)=\Omega_{\mathbf{d}}(n)-\mathcal{M}_{\mathbf{d}, Q}(n)
$$

and consider the sum

$$
\mathcal{E}(D, Q)=\sum_{(\mathcal{D})} \tau\left(d_{1}\right) \tau\left(d_{2}\right) \tau\left(d_{3}\right) \sum_{k \leq P}\left|\mathcal{G}_{\mathbf{d}, Q}\left(N-k^{2}\right)\right|
$$

where $\sum_{(\mathcal{D})}$ means that the summation is taken over squarefree odd integers $d_{1}, d_{2}, d_{3} \leq D$.

It is easy to see that $\mathcal{E}(D, Q) \ll P^{2+\varepsilon}$ for a large range of $D$ and $Q$. This simple estimate, however, is useless for our aims. To prove a non-trivial result we need an estimate of the shape (33) for a suitable $Q$ and for $D$ as large as possible. This is the most difficult part of the paper.

The following proposition holds:

Proposition 1. Suppose that

$$
Q=P^{20 / 23}, \quad D=P^{2 / 69-10 \varepsilon} .
$$

Then we have

$$
\mathcal{E}(D, Q) \ll P^{2-\varepsilon}
$$

This statement cannot be applied directly to our problem because of the complicated form of the main term. We will state another proposition, which is suitable for applying the sieve method.

Define

$$
\mathcal{L}_{\mathbf{d}}(N)=\sum_{\substack{p^{2}+x_{1}^{2}+x_{2}^{2}+x_{3}^{2}=N \\ x_{i} \equiv 0\left(d_{i}\right)}} \omega(\mathbf{x})
$$

Suppose that $\beta_{i}(d), i=1,2,3$, are real functions satisfying

$$
\beta_{i}(d)=0 \text { if } \mu(d)=0 \text { or } 2 \mid d
$$

and

$$
\left|\beta_{i}(d)\right| \leq \tau(d)
$$

Let

$$
\mathcal{H}(D)=\sum_{(\mathcal{D})} \beta_{1}\left(d_{1}\right) \beta_{2}\left(d_{2}\right) \beta_{3}\left(d_{3}\right)\left(\mathcal{L}_{\mathbf{d}}(N)-\frac{\mathcal{N}_{0}(N) \Sigma_{0}(\mathbf{d}, N)}{d_{1} d_{2} d_{3}}\right)
$$

where $\Sigma_{0}, \mathcal{N}_{0}$ and $\mathcal{L}_{\mathbf{d}}(N)$ are defined by (16), (26) and (34), respectively. 
Proposition 2. If $D=P^{2 / 69-10 \varepsilon}$ then we have

$$
\mathcal{H}(D) \ll P^{2}(\log P)^{-A} .
$$

At the beginning of the proof of Proposition 1 we will impose some simple restrictions on $Q$ and $D$ only. We will impose more severe restrictions later and we will explain the final choice (32) at the end of the proof.

For $\mathcal{M}_{\mathbf{d}, Q}(n)$ to be a good approximation to $\Omega_{\mathbf{d}}(n)$ we have to take $Q$ sufficiently large. However, it becomes difficult to work with $\mathcal{M}_{\mathbf{d}, Q}(n)$ if $Q$ is too large. Now we assume only that

$$
Q \leq P^{1-\varepsilon} .
$$

The reason for introducing this restriction is that in this case we are in a position to apply Lemma 1 with $x \asymp P$. Another reason is that the exponential sum $\mathcal{W}_{\mathbf{d}, Q}(\alpha)$, defined by (52), has a comparatively simple behaviour. This becomes clear from Lemma 16.

For $D$ we assume that

$$
D=P^{\alpha_{0}} \quad \text { where } \quad \alpha_{0} \in(0,1) .
$$

We note that estimates of the shape (33) and (38) with any small fixed $\alpha_{0}$ imply a nontrivial result for our additive problem. The result becomes better if $\alpha_{0}$ is larger, so our aim is to establish (33) and (38) with $\alpha_{0}$ as large as possible.

\subsection{Beginning of the proof of Proposition 1 .}

Consider the sum $\mathcal{E}(D, Q)$, defined by (31). We apply Cauchy's inequality to get

$$
\mathcal{E}^{2}(D, Q) \ll(\log P)^{12} P \mathcal{E}_{0},
$$

where

$$
\mathcal{E}_{0}=\sum_{(\mathcal{D})} d_{1} d_{2} d_{3} \sum_{k \leq P}\left|\mathcal{G}_{\mathbf{d}, Q}\left(N-k^{2}\right)\right|^{2} .
$$

To estimate this sum we apply the "square sieve", developed by the first author in [8]. We take

$$
R=P^{\alpha_{1}} \quad \text { where } \quad \alpha_{1} \in(0,1) .
$$

We shall specify the value of the constant $\alpha_{1}$ later. Consider the quantity

$$
\kappa(n, R)=\left(\frac{\log R}{R} \sum_{\substack{R<p \leq 2 R \\ p \nmid N}}\left(\frac{N-n}{p}\right)\right)^{2} .
$$

If $n=N-k^{2}$ for some integer $k \in(0, P]$ then $\kappa(n, R) \gg 1$ and, obviously, $\kappa(n, R) \geq 0$ for all $n$. Therefore we have

$$
\mathcal{E}_{0} \ll \sum_{(\mathcal{D})} d_{1} d_{2} d_{3} \sum_{n \in \mathbb{Z}} \kappa(n, R)\left|\mathcal{G}_{\mathbf{d}, Q}(n)\right|^{2} .
$$


We use the definition of $\kappa(n, R)$ to get

$$
\mathcal{E}_{0} \ll\left(\frac{\log R}{R}\right)^{2}\left|\mathcal{E}_{1}\right|+\frac{\log R}{R} \mathcal{E}_{2},
$$

where

$$
\begin{aligned}
& \mathcal{E}_{1}=\sum_{(\mathcal{D})} d_{1} d_{2} d_{3} \sum_{(\mathcal{R})} \sum_{n \in \mathbb{Z}}\left(\frac{N-n}{p p^{\prime}}\right)\left|\mathcal{G}_{\mathbf{d}, Q}(n)\right|^{2}, \\
& \mathcal{E}_{2}=\sum_{(\mathcal{D})} d_{1} d_{2} d_{3} \sum_{n \in \mathbb{Z}}\left|\mathcal{G}_{\mathbf{d}, Q}(n)\right|^{2} .
\end{aligned}
$$

From this point onwards $\sum_{(\mathcal{R})}$ means that the summation is taken over primes $p$ and $p^{\prime}$ such that $R<p, p^{\prime} \leq 2 R,\left(p p^{\prime}, N\right)=1$ and $p \neq p^{\prime}$.

\subsection{The estimation of the sum $\mathcal{E}_{2}$.}

\subsubsection{Preparation.}

We use (30) and (45) to represent $\mathcal{E}_{2}$ in the form

$$
\mathcal{E}_{2}=\mathcal{E}_{2}^{(1)}-2 \mathcal{E}_{2}^{(2)}+\mathcal{E}_{2}^{(3)}
$$

where $\mathcal{E}_{2}^{(i)}, i=1,2,3$, come from the consecutive terms in the expansion $|\mathcal{G}|^{2}=$ $\Omega^{2}-2 \Omega \mathcal{M}+\mathcal{M}^{2}$.

First we shall prove that

$$
\mathcal{E}_{2}^{(i)}=\sum_{(\mathcal{D})} d_{1} d_{2} d_{3} J_{2}^{(i)}, \quad i=1,2,3,
$$

where

$$
\begin{aligned}
& J_{2}^{(1)}=\int_{0}^{1}\left|f_{\mathbf{d}}(\alpha)\right|^{2} d \alpha \\
& J_{2}^{(2)}=\int_{0}^{1} f_{\mathbf{d}}(\alpha) \mathcal{W}_{\mathbf{d}, Q}(-\alpha) d \alpha \\
& J_{2}^{(3)}=\int_{0}^{1}\left|\mathcal{W}_{\mathbf{d}, Q}(\alpha)\right|^{2} d \alpha
\end{aligned}
$$

and where

$$
\begin{aligned}
f_{\mathbf{d}}(\alpha) & =\prod_{i=1}^{3} f_{d_{i}}(\alpha), \quad f_{d}(\alpha)=\sum_{\substack{x \in \mathbb{Z} \\
x \equiv 0(d)}} \omega(x) e\left(\alpha x^{2}\right), \\
\mathcal{W}_{\mathbf{d}, Q}(\alpha) & =\sum_{n \in \mathbb{Z}} \mathcal{M}_{\mathbf{d}, Q}(n) e(\alpha n) .
\end{aligned}
$$


The identity (47) for $\mathcal{E}_{2}^{(1)}$ is a consequence of the equalities

$$
\begin{aligned}
& \sum_{n \in \mathbb{Z}} \Omega_{\mathbf{d}}^{2}(n)=\sum_{\substack{x_{1}^{2}+x_{2}^{2}+x_{3}^{2}=y_{1}^{2}+y_{2}^{2}+y_{3}^{2} \\
x_{i}, y_{i} \equiv 0\left(d_{i}\right)}} \omega(\mathbf{x}) \omega(\mathbf{y}) \\
&= \sum_{\substack{\mathbf{x}, \mathbf{y} \in \mathbb{Z}^{3} \\
x_{i}, y_{i} \equiv 0\left(d_{i}\right)}} \omega(\mathbf{x}) \omega(\mathbf{y}) \int_{0}^{1} e\left(\alpha\left(x_{1}^{2}+x_{2}^{2}+x_{3}^{2}-y_{1}^{2}-y_{2}^{2}-y_{3}^{2}\right)\right) d \alpha \\
&=J_{2}^{(1)} .
\end{aligned}
$$

To prove the identity (47) for $\mathcal{E}_{2}^{(2)}$ we notice that

$$
\begin{aligned}
& \sum_{n \in \mathbb{Z}} \Omega_{\mathbf{d}}(n) \mathcal{M}_{\mathbf{d}, Q}(n) \\
& =\sum_{\substack{n \in \mathbb{Z}, \mathbf{x} \in \mathbb{Z}^{3} \\
x_{i}=0\left(d_{i}\right)}} \omega(\mathbf{x}) \mathcal{M}_{\mathbf{d}, Q}(n) \int_{0}^{1} e\left(\alpha\left(x_{1}^{2}+x_{2}^{2}+x_{3}^{2}-n\right)\right) d \alpha=J_{2}^{(2)} .
\end{aligned}
$$

Finally, the identity (47) for $\mathcal{E}_{2}^{(3)}$ is a consequence of

$$
\sum_{n \in \mathbb{Z}} \mathcal{M}_{\mathbf{d}, Q}^{2}(n)=\int_{0}^{1}\left|\mathcal{W}_{\mathbf{d}, Q}(\alpha)\right|^{2} d \alpha
$$

\subsubsection{An asymptotic formula for $\mathcal{E}_{2}^{(1)}$.}

Consider the integral $J_{2}^{(1)}$ defined by (48). We study it by means of the Kloosterman method [16]. We consider the Farey dissection of order $P$ for the unit interval and find that

$$
J_{2}^{(1)}=\sum_{q \leq P} \sum_{a(q)^{*}} \int_{\mathcal{B}(q, a)}\left|f_{\mathbf{d}}\left(\frac{a}{q}+\beta\right)\right|^{2} d \beta
$$

where

$$
\mathcal{B}(q, a)=\left[-\left(q\left(q+q^{\prime}\right)\right)^{-1},\left(q\left(q+q^{\prime \prime}\right)\right)^{-1}\right]
$$

and where $q^{\prime}$ and $q^{\prime \prime}$ are defined by

$$
P<q+q^{\prime}, q+q^{\prime \prime} \leq q+P, \quad q+q^{\prime} \equiv \bar{a}(q), \quad q+q^{\prime \prime} \equiv-\bar{a}(q) .
$$

We shall find an expression for the integrand in the right hand side of (53) in which the variables $a$ and $\beta$ are separated. The following Lemma holds:

Lemma 12. Suppose that $q, d, b \in \mathbb{N}, h \in \mathbb{Z}, \beta \in \mathbb{R}, q \leq P,|\beta| \leq(q P)^{-1}$ and $d, b \leq P^{c}$ for some constant $c>0$. Then for any constant $\gamma \geq 1$ we have

$$
f_{d}\left(\frac{h}{b}+\beta\right)=\frac{P}{b d} \sum_{|n| \leq \gamma b d q^{-1} P^{\varepsilon}} S\left(b, h d^{2}, n\right) I\left(\beta N,-\frac{P n}{b d}\right)+\mathcal{O}\left(P^{-A}\right) .
$$

The constant in the $\mathcal{O}$ - term depends on $c, A$ and $\varepsilon$. 
Proof: Using (51) we get

$$
f_{d}\left(\frac{h}{b}+\beta\right)=\sum_{y \in \mathbb{Z}} \omega(d y) e_{b}\left(h d^{2} y^{2}\right) e\left(\beta d^{2} y^{2}\right)=\sum_{m(b)} e_{b}\left(h d^{2} m^{2}\right) \mathcal{Z}_{m}
$$

where

$$
\mathcal{Z}_{m}=\sum_{\substack{y \in \mathbb{Z} \\ y \equiv m(b)}} \omega(d y) e\left(\beta d^{2} y^{2}\right) .
$$

Consider $\mathcal{Z}_{m}$. We apply Poisson's formula and use (18) and (19) to get

$$
\begin{aligned}
\mathcal{Z}_{m} & =\sum_{x \in \mathbb{Z}} \omega(d(m+b x)) e\left(\beta d^{2}(m+b x)^{2}\right) \\
& =\sum_{n \in \mathbb{Z}} \int_{-\infty}^{\infty} \omega(d(m+b x)) e\left(\beta d^{2}(m+b x)^{2}\right) e(-n x) d x \\
& =\frac{P}{b d} \sum_{n \in \mathbb{Z}} e_{b}(n m) I\left(\beta N,-\frac{n P}{b d}\right) .
\end{aligned}
$$

The absolute convergence of the last series is an easy consequence of Lemma 9 (i). Indeed, if $n \neq 0$ then we have

$$
I\left(\beta N,-\frac{n P}{b d}\right) \ll\left(1+|\beta|^{k} N^{k}\right)\left(\frac{|n| P}{b d}\right)^{-k} \ll\left(\frac{b d}{|n| q}\right)^{k} .
$$

Here $k$ can be an arbitrarily large integer and the constant in the $\ll-$ symbol depends on $k$.

We use (6), (56) and (57) to get

$$
f_{d}\left(\frac{h}{b}+\beta\right)=\frac{P}{b d} \sum_{n \in \mathbb{Z}} S\left(b, h d^{2}, n\right) I\left(\beta N,-\frac{n P}{b d}\right) .
$$

It remains to estimate the contribution $\mathcal{X}$ arising from the terms with $|n|>$ $\gamma b d q^{-1} P^{\varepsilon}$. We take $k=\left[\varepsilon^{-1}(2 c+A+3)\right]$ and apply (6) and (58). We use the elementary inequality $\sum_{n>\alpha} n^{-k} \leq \alpha^{-k}+\alpha^{-k+1}$, which holds true for $\alpha>0$ and for $k \geq 2$. We conclude that $\mathcal{X} \ll P^{-A}$ and this proves the Lemma.

We use (8), (19), (20) and Lemmas $2(i i)$ and 12 to find that the integrand in the right hand side of (53) equals

$$
\begin{aligned}
\frac{P^{6}}{q^{6}\left(d_{1} d_{2} d_{3}\right)^{2}} & \sum_{\mathbf{n} \in \mathcal{N}_{\mathbf{d}, q}} \sum_{\mathbf{l} \in \mathcal{N}_{\mathbf{d}, q}} S_{\mathbf{d}}(q, a, \mathbf{n}) S_{\mathbf{d}}(q,-a,-\mathbf{l}) \\
& I_{\mathbf{d}}\left(\beta N,-P q^{-1} \mathbf{n}\right) I_{\mathbf{d}}\left(-\beta N, P q^{-1} \mathbf{l}\right)+\mathcal{O}\left(P^{-A}\right),
\end{aligned}
$$


where we have set

(60) $\mathcal{N}_{\mathbf{d}, q}(H)=\left\{\mathbf{n} \in \mathbb{Z}^{3}:\left|n_{i}\right| \leq 4 d_{i} H P^{\varepsilon}, n_{i} \equiv 0\left(\left(q, d_{i}^{2}\right)\right), i=1,2,3\right\}$,

(61) $\quad \mathcal{N}_{\mathbf{d}, q}=\mathcal{N}_{\mathbf{d}, q}(1)$.

We use (47), (53) and (59) and find an expression for $\mathcal{E}_{2}^{(1)}$. Then we change the variable $\beta N=\beta^{\prime}$ and denote

$$
\mathcal{B}^{\prime}(q, a)=\left[-N\left(q\left(q+q^{\prime}\right)\right)^{-1}, N\left(q\left(q+q^{\prime \prime}\right)\right)^{-1}\right] .
$$

We obtain

$$
\begin{aligned}
\mathcal{E}_{2}^{(1)}= & P^{4} \sum_{(\mathcal{D})}\left(d_{1} d_{2} d_{3}\right)^{-1} \sum_{q \leq P} q^{-6} \sum_{\mathbf{n} \in \mathcal{N}_{\mathbf{d}, q}} \sum_{\mathbf{l} \in \mathcal{N}_{\mathbf{d}, q}} \\
& \times \sum_{a(q)^{*}} S_{\mathbf{d}}(q, a, \mathbf{n}) S_{\mathbf{d}}(q,-a,-\mathbf{l}) \mathcal{T}_{\mathbf{d}}(\mathbf{n}, \mathbf{l}, q, a)+\mathcal{O}\left(P^{-A}\right),
\end{aligned}
$$

where

(64)

$$
\mathcal{T}_{\mathbf{d}}(\mathbf{n}, \mathbf{l}, q, a)=\int_{\mathcal{B}^{\prime}(q, a)} I_{\mathbf{d}}\left(\beta,-P q^{-1} \mathbf{n}\right) I_{\mathbf{d}}\left(-\beta, P q^{-1} \mathbf{l}\right) d \beta
$$

Define

$$
\lambda(\mathbf{d}, \mathbf{n}, \mathbf{l}, H)= \begin{cases}\sum_{i=1}^{3} d_{i}^{-2}\left(n_{i}^{2} H^{-2}+l_{i}^{2}\right) & \text { if }(\mathbf{n}, \mathbf{l}) \neq(\mathbf{0}, \mathbf{0}) \\ 1 & \text { otherwise }\end{cases}
$$

and let

$$
\lambda(\mathbf{d}, \mathbf{n}, \mathbf{l})=\lambda(\mathbf{d}, \mathbf{n}, \mathbf{l}, 1) .
$$

We appeal to $(23),(55),(62)$, Lemma 9 (iii) and Lemma $10(i)$ to get

$$
\mathcal{T}_{\mathbf{d}}(\mathbf{n}, \mathbf{l}, q, a)= \begin{cases}\mathcal{O}\left(q^{2} P^{-2+2 \varepsilon} \lambda(\mathbf{d}, \mathbf{n}, \mathbf{l})^{-1}\right) & \text { if } \quad(\mathbf{n}, \mathbf{l}) \neq(\mathbf{0}, \mathbf{0}) \\ \kappa_{0}+\mathcal{O}\left(q^{2} P^{-2}\right) & \text { otherwise }\end{cases}
$$

From Lemma 2 (ii) and (iii) we find that

$$
\sum_{a(q)^{*}}\left|S_{\mathbf{d}}(q, a, \mathbf{n}) S_{\mathbf{d}}(q,-a,-\mathbf{l})\right| \ll q^{4} \xi(q, \mathbf{d}),
$$

where

$$
\xi(q, \mathbf{d})=\prod_{i=1}^{3}\left(q, d_{i}^{2}\right) .
$$

We use (63), (67) - (69) to obtain

$$
\mathcal{E}_{2}^{(1)}=\mathcal{U}_{2}+\mathcal{O}\left(P^{2+2 \varepsilon} \mathcal{Y}(P)\right)
$$


where

$$
\mathcal{U}_{2}=\kappa_{0} P^{4} \sum_{(\mathcal{D})}\left(d_{1} d_{2} d_{3}\right)^{-1} \sum_{q \leq P} q^{-6} \sum_{a(q)^{*}}\left|S_{\mathbf{d}}(q, a)\right|^{2}
$$

and

$$
\mathcal{Y}(K)=\sum_{q \leq K} \sum_{(\mathcal{D})} \sum_{\mathbf{n} \in \mathcal{N}_{\mathbf{d}, q}} \sum_{\mathbf{l} \in \mathcal{N}_{\mathbf{d}, q}} \frac{\xi(q, \mathbf{d})}{d_{1} d_{2} d_{3} \lambda(\mathbf{d}, \mathbf{n}, \mathbf{l})}
$$

It remains to estimate $\mathcal{Y}$. We first prove two simple results

Lemma 13. Suppose that $d_{1}, d_{2}, d_{3} \leq D$. Then we have

$$
\sum_{\mathbf{l} \in \mathcal{N}_{\mathbf{d}, 1}} \lambda(\mathbf{d}, \mathbf{0}, \mathbf{l})^{-1} \ll D^{3} P^{\varepsilon} .
$$

Proof: The sum under consideration is $\ll 1+T_{1}+T_{2}+T_{3}$, where $T_{\nu}$ is the contribution from $\mathbf{l}$ such that $\left|l_{\nu}\right| d_{\nu}^{-1}=\max _{i=1,2,3}\left(\left|l_{i}\right| d_{i}^{-1}\right)>0$. Consider, for example, $T_{1}$. We have

$$
T_{1} \ll \sum_{1 \leq l_{1} \leq d_{1} P^{\varepsilon}} \frac{d_{1}^{2}}{l_{1}^{2}}\left(1+\frac{d_{2} l_{1}}{d_{1}}\right)\left(1+\frac{d_{3} l_{1}}{d_{1}}\right) \ll D^{3} P^{\varepsilon} .
$$

This proves the Lemma.

Lemma 14. Let $q \leq P$. The following estimate holds:

$$
\sum_{d \leq D} \frac{\left(q, d^{2}\right)}{d} \ll D P^{\varepsilon}
$$

Proof: We have

$$
\sum_{d \leq D} \frac{\left(q, d^{2}\right)}{d} \leq \sum_{d \leq D} \frac{(q, d)^{2}}{d} \leq \sum_{d \leq D}(q, d) \ll D P^{\varepsilon} .
$$

We are now in a position to estimate $\mathcal{Y}$.

Lemma 15. Suppose that $K \leq P$. For the sum $\mathcal{Y}(K)$, defined by (72), we have

$$
\mathcal{Y}(K) \ll K D^{6} P^{7 \varepsilon} .
$$


Proof: It is clear that $\mathcal{Y}(K) \ll \mathcal{Y}^{\prime}+\mathcal{Y}^{\prime \prime}$, where $\mathcal{Y}^{\prime}$ is the contribution of the terms with $\mathbf{l}=\mathbf{0}$ and $\mathcal{Y}^{\prime \prime}$ is the contribution of the other terms.

Consider $\mathcal{Y}^{\prime \prime}$. Using Lemma 13 we get

$$
\sum_{\substack{\mathbf{l} \in \mathcal{N}_{\mathbf{d}, q} \\ \mathbf{l} \neq \mathbf{0}}} \lambda(\mathbf{d}, \mathbf{n}, \mathbf{l})^{-1} \ll \sum_{\mathbf{l} \in \mathcal{N}_{\mathbf{d}, q}} \lambda(\mathbf{d}, \mathbf{0}, \mathbf{l})^{-1} \ll D^{3} P^{\varepsilon}
$$

and, obviously,

$$
\sum_{\mathbf{n} \in \mathcal{N}_{\mathbf{d}, q}} 1 \ll P^{3 \varepsilon} \prod_{i=1}^{3}\left(1+\frac{d_{i}}{\left(q, d_{i}^{2}\right)}\right) .
$$

Now we use (69) to get

$$
\mathcal{Y}^{\prime \prime} \ll D^{3} P^{4 \varepsilon} \sum_{q \leq K}\left(\sum_{d \leq D} \frac{\left(q, d^{2}\right)}{d}\left(1+\frac{d}{\left(q, d^{2}\right)}\right)\right)^{3} .
$$

To estimate the sum over $d$ we apply Lemma 14 and find that $\mathcal{Y}^{\prime \prime} \ll K D^{6} P^{7 \varepsilon}$.

Consider now $\mathcal{Y}^{\prime}$. We apply Lemma 13 with the rôles of $\mathbf{n}$ and $\mathbf{l}$ reversed and get $\sum_{\mathbf{n} \in \mathcal{N}_{\mathbf{d}, q}} \lambda(\mathbf{d}, \mathbf{n}, \mathbf{0})^{-1} \ll D^{3} P^{\varepsilon}$. Hence

$$
\mathcal{Y}^{\prime} \ll D^{3} P^{\varepsilon} \sum_{q \leq K}\left(\sum_{d \leq D} \frac{\left(q, d^{2}\right)}{d}\right)^{3} .
$$

Now we use Lemma 14 again and obtain $\mathcal{Y}^{\prime} \ll K D^{6} P^{4 \varepsilon}$. This proves the Lemma.

From (70) and Lemma 15 we obtain

$$
\mathcal{E}_{2}^{(1)}=\mathcal{U}_{2}+\mathcal{O}\left(P^{3+9 \varepsilon} D^{6}\right) .
$$

\subsubsection{An asymptotic formula for $\mathcal{E}_{2}^{(2)}$.}

Consider the integral $J_{2}^{(2)}$, defined by (49). Using the Farey decomposition of order $P$ of the unit interval we get

$$
J_{2}^{(2)}=\sum_{q \leq P} \sum_{a(q)^{*}} \int_{\mathcal{B}(q, a)} f_{\mathbf{d}}\left(\frac{a}{q}+\beta\right) \mathcal{W}_{\mathbf{d}, Q}\left(-\frac{a}{q}-\beta\right) d \beta .
$$

As in the previous section, we first separate the terms involving $a$ and $\beta$. For $f_{\mathbf{d}}$ we apply Lemma 12 . To deal with $\mathcal{W}_{\mathbf{d}, Q}$ we use the following 
Lemma 16. Suppose that $Q \leq P^{1-\varepsilon},|\beta| \leq(q P)^{-1}$ and $(a, q)=1$. Then we have

$$
\mathcal{W}_{\mathbf{d}, Q}\left(\frac{a}{q}+\beta\right)= \begin{cases}\frac{P^{3}}{q^{3} d_{1} d_{2} d_{3}} S_{\mathbf{d}}(q, a) I^{3}(\beta N)+\mathcal{O}\left(P^{-A}\right) & \text { if } q \leq Q, \\ \mathcal{O}\left(P^{-A}\right) & \text { if } Q<q \leq P .\end{cases}
$$

Proof: We use definitions (29) and (52) of $\mathcal{M}_{\mathbf{d}, Q}$ and $\mathcal{W}_{\mathbf{d}, Q}$, respectively, to get

$$
\mathcal{W}_{\mathbf{d}, Q}\left(\frac{a}{q}+\beta\right)=\frac{P}{d_{1} d_{2} d_{3}} \sum_{q_{1} \leq Q} q_{1}^{-3} \sum_{a_{1}\left(q_{1}\right)^{*}} S_{\mathbf{d}}\left(q_{1}, a_{1}\right) \Phi,
$$

where

$$
\Phi=\sum_{n \in \mathbb{Z}} H\left(\frac{n}{N}\right) e\left(\left(\frac{a}{q}-\frac{a_{1}}{q_{1}}+\beta\right) n\right) .
$$

We apply the Poisson formula, change the variable in the integrals and then apply Lemma 11:

$$
\begin{aligned}
\Phi & =N \sum_{m \in \mathbb{Z}} \int_{-\infty}^{\infty} H(x) e\left(\left(\frac{a}{q}-\frac{a_{1}}{q_{1}}+\beta-m\right) N x\right) d x \\
& =N \sum_{m \in \mathbb{Z}} I^{3}\left(\left(\frac{a}{q}-\frac{a_{1}}{q_{1}}+\beta-m\right) N\right) .
\end{aligned}
$$

We may suppose that $0 \leq a \leq q-1$ and $0 \leq a_{1} \leq q_{1}-1$. In this case we have

$$
\left|\frac{a}{q}-\frac{a_{1}}{q_{1}}+\beta\right| \leq 1-\frac{1}{2 P} .
$$

We apply Lemma 9 (ii) and easily find that the contribution to $\Phi$ coming from $m \neq 0$ is $\mathcal{O}\left(P^{-A}\right)$.

Consider the term corresponding to $m=0$. Suppose that $a / q \neq a_{1} / q_{1}$. We have

$$
\left|\left(\frac{a}{q}-\frac{a_{1}}{q_{1}}+\beta\right) N\right| \geq \frac{\left|a q_{1}-a_{1} q\right|}{q q_{1}} N-|\beta| N \geq \frac{N}{q q_{1}}-\frac{N}{q P} \geq \frac{1}{2} P^{\varepsilon} .
$$

We use Lemma 9 (ii) again and find that in this case $I^{3}\left(\left(\frac{a}{q}-\frac{a_{1}}{q_{1}}+\beta\right) N\right) \ll$ $P^{-A}$

If $Q<q \leq P$ then there is no fraction $a_{1} / q_{1}$ equal to $a / q$.

If $q \leq Q$ then there is exactly one fraction $a_{1} / q_{1}$ equal to $a / q$, namely $a_{1}=a, q_{1}=q$. This proves the Lemma. 
Using (39) and Lemmas 12 and 16 we find that if $q \leq Q$ then the integrand in the right-hand side of (74) equals

$$
\begin{aligned}
\frac{P^{6}}{q^{6}\left(d_{1} d_{2} d_{3}\right)^{2}} & \sum_{\mathbf{n} \in \mathcal{N}_{\mathbf{d}, q}} S_{\mathbf{d}}(q, a, \mathbf{n}) S_{\mathbf{d}}(q,-a) \\
& I_{\mathbf{d}}\left(\beta N,-P q^{-1} \mathbf{n}\right) I^{3}(-\beta N)+\mathcal{O}\left(P^{-A}\right) .
\end{aligned}
$$

If $Q<q \leq P$ then the integrand is $\mathcal{O}\left(P^{-A}\right)$. Hence, using (47), (64) and (74) we find

$$
\begin{aligned}
\mathcal{E}_{2}^{(2)}= & P^{4} \sum_{(\mathcal{D})}\left(d_{1} d_{2} d_{3}\right)^{-1} \sum_{q \leq Q} q^{-6} \sum_{\mathbf{n} \in \mathcal{N}_{\mathbf{d}, q}} \\
& \times \sum_{a(q)^{*}} S_{\mathbf{d}}(q, a, \mathbf{n}) S_{\mathbf{d}}(q,-a) \mathcal{T}_{\mathbf{d}}(\mathbf{n}, \mathbf{0}, q, a)+\mathcal{O}\left(P^{-A}\right) .
\end{aligned}
$$

Now we use (67) - (69) and Lemma 15 to get

$$
\mathcal{E}_{2}^{(2)}=\mathcal{U}_{2}^{*}+\mathcal{O}\left(P^{3+9 \varepsilon} D^{6}\right),
$$

where

$$
\mathcal{U}_{2}^{*}=\kappa_{0} P^{4} \sum_{(\mathcal{D})}\left(d_{1} d_{2} d_{3}\right)^{-1} \sum_{q \leq Q} q^{-6} \sum_{a(q)^{*}}\left|S_{\mathbf{d}}(q, a)\right|^{2} .
$$

Let us compare $\mathcal{U}_{2}^{*}$ with $\mathcal{U}_{2}$, defined by (71). We use (68), (69), (71), (76) and Lemma 14 to get

$$
\begin{aligned}
\mathcal{U}_{2}-\mathcal{U}_{2}^{*} & \ll P^{4} \sum_{(\mathcal{D})}\left(d_{1} d_{2} d_{3}\right)^{-1} \sum_{Q<q \leq P} q^{-6} \sum_{a(q)^{*}}\left|S_{\mathbf{d}}(q, a)\right|^{2} \\
& \ll P^{4} \sum_{(\mathcal{D})}\left(d_{1} d_{2} d_{3}\right)^{-1} \sum_{Q<q \leq P} q^{-2} \xi(q, \mathbf{d}) \\
& \ll P^{4} \sum_{Q<q \leq P} q^{-2}\left(\sum_{d \leq D} \frac{\left(q, d^{2}\right)}{d}\right)^{3} \\
& \ll P^{4+3 \varepsilon} D^{3} \sum_{Q<q} q^{-2} \ll P^{4+3 \varepsilon} D^{3} Q^{-1} .
\end{aligned}
$$

Formulas (75) and (77) imply

$$
\mathcal{E}_{2}^{(2)}=\mathcal{U}_{2}+\mathcal{O}\left(\left(P^{3} D^{6}+P^{4} D^{3} Q^{-1}\right) P^{9 \varepsilon}\right) .
$$

\subsubsection{An asymptotic formula for $\mathcal{E}_{2}^{(3)}$.}

For the integral $J_{2}^{(3)}$, defined by (50), we have

$$
J_{2}^{(3)}=\sum_{q \leq P} \sum_{a(q)^{*}} \int_{\mathcal{B}(q, a)}\left|\mathcal{W}_{\mathbf{d}, Q}\left(\frac{a}{q}+\beta\right)\right|^{2} d \beta .
$$


Using (39), (47), (79) and Lemma 16 we obtain

$$
\begin{aligned}
\mathcal{E}_{2}^{(3)}=P^{6} & \sum_{(\mathcal{D})}\left(d_{1} d_{2} d_{3}\right)^{-1} \sum_{q \leq Q} q^{-6} \\
& \times \sum_{a(q)^{*}}\left|S_{\mathbf{d}}(q, a)\right|^{2} \int_{\mathcal{B}(q, a)}|I(\beta N)|^{6} d \beta+\mathcal{O}\left(P^{-A}\right) .
\end{aligned}
$$

Now we change the variable in the integral and use (64) and (67). Then we proceed as in the previous section to get

$$
\begin{aligned}
\mathcal{E}_{2}^{(3)} & =\mathcal{U}_{2}^{*}+\mathcal{O}\left(P^{3+9 \varepsilon} D^{6}\right) \\
& =\mathcal{U}_{2}+\mathcal{O}\left(\left(P^{3} D^{6}+P^{4} D^{3} Q^{-1}\right) P^{9 \varepsilon}\right) .
\end{aligned}
$$

\subsubsection{The estimate for $\mathcal{E}_{2}$.}

From (46), (73), (78) and (81) we obtain

$$
\mathcal{E}_{2} \ll\left(P^{3} D^{6}+P^{4} D^{3} Q^{-1}\right) P^{9 \varepsilon} .
$$

Consider the inequalities (41), (43) and (82). It is clear that in order to obtain (33) we must impose further restrictions on $D, Q$ and $R$. From this point onwards we assume that

$$
D \leq R^{1 / 6} P^{-10 \varepsilon}, \quad P^{1+20 \varepsilon} D^{3} R^{-1} \leq Q .
$$

\subsection{The estimation of the sum $\mathcal{E}_{1}$.}

\subsubsection{Preparation.}

Consider the sum $\mathcal{E}_{1}$, defined by (44). We represent it in the form

$$
\mathcal{E}_{1}=\mathcal{E}_{1}^{(1)}-2 \mathcal{E}_{1}^{(2)}+\mathcal{E}_{1}^{(3)}
$$

where $\mathcal{E}_{1}^{(i)}$ are the contributions of the consecutive terms from the expansion $|\mathcal{G}|^{2}=\Omega^{2}-2 \Omega \mathcal{M}+\mathcal{M}^{2}$.

First we shall prove that for the sums $\mathcal{E}_{1}^{(i)}$ the following formulas hold:

$$
\mathcal{E}_{1}^{(i)}=\sum_{(\mathcal{R})} \frac{\gamma\left(p p^{\prime}\right)}{p p^{\prime}} \sum_{(\mathcal{D})} d_{1} d_{2} d_{3} \sum_{s\left(p p^{\prime}\right)^{*}}\left(\frac{s}{p p^{\prime}}\right) e_{p p^{\prime}}(-s N) J_{1}^{(i)}, \quad i=1,2,3,
$$

where $\gamma\left(p p^{\prime}\right)$ is the Gauss sum,defined by (5), and

$$
\begin{aligned}
& J_{1}^{(1)}=\int_{0}^{1} f_{\mathbf{d}}\left(\alpha+\frac{s}{p p^{\prime}}\right) f_{\mathbf{d}}(-\alpha) d \alpha, \\
& J_{1}^{(2)}=\int_{0}^{1} f_{\mathbf{d}}\left(\alpha+\frac{s}{p p^{\prime}}\right) \mathcal{W}_{\mathbf{d}}(-\alpha) d \alpha, \\
& J_{1}^{(3)}=\sum_{n \in \mathbb{Z}} \mathcal{M}_{\mathbf{d}, Q}^{2}(n) e_{p p^{\prime}}(s n) .
\end{aligned}
$$


Consider the sum $\mathcal{E}_{1}^{(1)}$. We have

$$
\begin{aligned}
\mathcal{E}_{1}^{(1)} & =\sum_{(\mathcal{R})} \sum_{(\mathcal{D})} d_{1} d_{2} d_{3} \sum_{n \in \mathbb{Z}}\left(\frac{N-n}{p p^{\prime}}\right) \Omega_{\mathbf{d}}^{2}(n) \\
& =\sum_{(\mathcal{R})} \sum_{(\mathcal{D})} d_{1} d_{2} d_{3} \Gamma_{1}^{(1)},
\end{aligned}
$$

say. Obviously, (28) implies

$$
\Gamma_{1}^{(1)}=\sum_{\substack{x_{1}^{2}+x_{2}^{2}+x_{3}^{2}=y_{1}^{2}+y_{2}^{2}+y_{3}^{2} \\ x_{i}, y_{i} \equiv 0\left(d_{i}\right)}} \omega(\mathbf{x}) \omega(\mathbf{y})\left(\frac{N-\left(x_{1}^{2}+x_{2}^{2}+x_{3}^{2}\right)}{p p^{\prime}}\right) .
$$

Using (51) and taking into account the elementary properties of the Gauss sum $\gamma(q)$ we get

$$
\begin{aligned}
\Gamma_{1}^{(1)}= & \sum_{l\left(p p^{\prime}\right)}\left(\frac{l}{p p^{\prime}}\right) \sum_{\substack{x_{1}^{2}+x_{2}^{2}+x_{3}^{2}=y_{1}^{2}+y_{2}^{2}+y_{3}^{2} \\
x_{1}^{2}+x_{2}^{2}+x_{3}^{2} \equiv N-l\left(p p^{\prime}\right) \\
x_{i}, y_{i} \equiv 0\left(d_{i}\right)}} \omega(\mathbf{x}) \omega(\mathbf{y}) \\
= & \sum_{l\left(p p^{\prime}\right)}\left(\frac{l}{p p^{\prime}}\right) \sum_{\substack{\mathbf{x}, \mathbf{y} \in \mathbb{Z}^{3} \\
x_{i}, y_{i} \equiv 0\left(d_{i}\right)}} \omega(\mathbf{x}) \omega(\mathbf{y}) \\
& \times \int_{0}^{1} e\left(\alpha\left(x_{1}^{2}+x_{2}^{2}+x_{3}^{2}-y_{1}^{2}-y_{2}^{2}-y_{3}^{2}\right)\right) d \alpha \\
& \times \frac{1}{p p^{\prime}} \sum_{s\left(p p^{\prime}\right)} e_{p p^{\prime}}\left(s\left(x_{1}^{2}+x_{2}^{2}+x_{3}^{2}-N+l\right)\right) \\
= & \frac{1}{p p^{\prime}} \sum_{l\left(p p^{\prime}\right)}\left(\frac{l}{p p^{\prime}}\right) \sum_{s\left(p p^{\prime}\right)} e_{p p^{\prime}}(s(l-N)) J_{1}^{(1)} \\
= & \frac{\gamma\left(p p^{\prime}\right)}{p p^{\prime}} \sum_{s\left(p p^{\prime}\right)^{*}}\left(\frac{s}{p p^{\prime}}\right) e_{p p^{\prime}}(-s N) J_{1}^{(1)},
\end{aligned}
$$

where $J_{1}^{(1)}$ is defined by (86). From (89) and (90) we obtain formula (85) for $\mathcal{E}_{1}^{(1)}$.

Consider $\mathcal{E}_{1}^{(2)}$. We have

$$
\begin{aligned}
\mathcal{E}_{1}^{(2)} & =\sum_{(\mathcal{R})} \sum_{(\mathcal{D})} d_{1} d_{2} d_{3} \sum_{n \in \mathbb{Z}}\left(\frac{N-n}{p p^{\prime}}\right) \Omega_{\mathbf{d}}(n) \mathcal{M}_{\mathbf{d}, Q}(n) \\
& =\sum_{(\mathcal{R})} \sum_{(\mathcal{D})} d_{1} d_{2} d_{3} \Gamma_{1}^{(2)},
\end{aligned}
$$


say. Furthermore

$$
\Gamma_{1}^{(2)}=\sum_{l\left(p p^{\prime}\right)}\left(\frac{l}{p p^{\prime}}\right) \sum_{\substack{n \in \mathbb{Z} \\ n \equiv N-l\left(p p^{\prime}\right)}} \Omega_{\mathbf{d}}(n) \mathcal{M}_{\mathbf{d}, Q}(n)=\sum_{l\left(p p^{\prime}\right)}\left(\frac{l}{p p^{\prime}}\right) \Phi^{*},
$$

say. Obviously

$$
\Phi^{*}=\sum_{\substack{n \in \mathbb{Z}, \mathbf{x} \in \mathbb{Z}^{3} \\ x_{1}^{2}+x_{2}^{2}+x_{3}^{2}=n \equiv N-l\left(p p^{\prime}\right) \\ x_{i} \equiv 0\left(d_{i}\right)}} \omega(\mathbf{x}) \mathcal{M}_{\mathbf{d}, Q}(n) .
$$

By the previous formula and (51), (52) we obtain

$$
\begin{aligned}
\Phi^{*}= & \sum_{\substack{n \in \mathbb{Z}, \mathbf{x} \in \mathbb{Z}^{3} \\
x_{i} \equiv 0\left(d_{i}\right)}} \omega(\mathbf{x}) \mathcal{M}_{\mathbf{d}, Q}(n) \int_{0}^{1} e\left(\alpha\left(x_{1}^{2}+x_{2}^{2}+x_{3}^{2}-n\right)\right) d \alpha \\
& \times \frac{1}{p p^{\prime}} \sum_{s\left(p p^{\prime}\right)} e_{p p^{\prime}}\left(s\left(x_{1}^{2}+x_{2}^{2}+x_{3}^{2}-N+l\right)\right) \\
= & \frac{1}{p p^{\prime}} \sum_{s\left(p p^{\prime}\right)} e_{p p^{\prime}}(s(l-N)) J_{1}^{(2)}
\end{aligned}
$$

where $J_{1}^{(2)}$ is defined by (87). It remains to substitute this expression for $\Phi^{*}$ in (92) and to change the order of summation over $l$ and $s$. The Gauss sum $\gamma(q)$ appears again. We use (91) and obtain formula (85) for $\mathcal{E}_{1}^{(2)}$.

Consider $\mathcal{E}_{1}^{(3)}$. We have

$$
\begin{aligned}
\mathcal{E}_{1}^{(3)} & =\sum_{(\mathcal{R})} \sum_{(\mathcal{D})} d_{1} d_{2} d_{3} \sum_{n \in \mathbb{Z}}\left(\frac{N-n}{p p^{\prime}}\right) \mathcal{M}_{\mathbf{d}, Q}^{2}(n) \\
& =\sum_{(\mathcal{R})} \sum_{(\mathcal{D})} d_{1} d_{2} d_{3} \Gamma_{1}^{(3)}
\end{aligned}
$$

say. It is clear that

$$
\begin{aligned}
\Gamma_{1}^{(3)} & =\sum_{l\left(p p^{\prime}\right)}\left(\frac{l}{p p^{\prime}}\right) \sum_{\substack{n \in \mathbb{Z} \\
n \equiv N-l\left(p p^{\prime}\right)}} \mathcal{M}_{\mathbf{d}, Q}^{2}(n) \\
& =\sum_{l\left(p p^{\prime}\right)}\left(\frac{l}{p p^{\prime}}\right) \sum_{n \in \mathbb{Z}} \mathcal{M}_{\mathbf{d}, Q}^{2}(n) \frac{1}{p p^{\prime}} \sum_{s\left(p p^{\prime}\right)} e_{p p^{\prime}}(s(n-N+l)) \\
& =\frac{\gamma\left(p p^{\prime}\right)}{p p^{\prime}} \sum_{s\left(p p^{\prime}\right)^{*}}\left(\frac{s}{p p^{\prime}}\right) e_{p p^{\prime}}(-s N) J_{1}^{(3)},
\end{aligned}
$$

where $J_{1}^{(3)}$ is specified by (88). From (93) and (94) we obtain formula (85) for $\mathcal{E}_{1}^{(3)}$ 


\subsubsection{The sum $\mathcal{E}_{1}^{(1)}$.}

In this section we will establish the asymptotic formula (109). Its remainder term, however, is still a complicated expression which we shall estimate later.

Consider the integral $J_{1}^{(1)}$, defined by (86). We represent again the unit interval as an union of Farey arcs

$$
\bigcup_{q \leq P} \bigcup_{\substack{1 \leq a \leq q \\(a, q)=1}}(\mathcal{B}(q, a)+a / q),
$$

where $\mathcal{B}(q, a)$ is defined by (54). We decompose further the set of integration according to the size of $\left(a p p^{\prime}+s q, q p p^{\prime}\right)$. We note that $\left(a p p^{\prime}+s q, q p p^{\prime}\right) \mid\left(p p^{\prime}\right)^{2}$ and find

$$
\begin{aligned}
& J_{1}^{(1)}=\sum_{\delta \mid\left(p p^{\prime}\right)^{2}} \sum_{q \leq P} \\
& \times \sum_{\substack{a(q)^{*} \\
\left(a p p^{\prime}+s q, q p p^{\prime}\right)=\delta}} \int_{\mathcal{B}(q, a)} f_{\mathbf{d}}\left(\frac{a}{q}+\frac{s}{p p^{\prime}}+\beta\right) f_{\mathbf{d}}\left(-\frac{a}{q}-\beta\right) d \beta .
\end{aligned}
$$

We use Lemmas $2(i i)$ and 12 to see that the integrand in the last formula equals

$$
\begin{aligned}
\frac{P^{6} \delta^{3}}{q^{6}\left(p p^{\prime}\right)^{3}\left(d_{1} d_{2} d_{3}\right)^{2}} & \sum_{\mathbf{n} \in \mathcal{N}_{\mathbf{d}, q}\left(R^{2} \delta^{-1}\right)} \sum_{\mathbf{l} \in \mathcal{N}_{\mathbf{d}, q}} \\
& \times S_{\mathbf{d}}\left(\frac{q p p^{\prime}}{\delta}, \frac{a p p^{\prime}+s q}{\delta}, \mathbf{n}\right) S_{\mathbf{d}}(q,-a,-\mathbf{l}) \\
& \times I_{\mathbf{d}}\left(\beta N,-\frac{P \delta}{q p p^{\prime}} \mathbf{n}\right) I_{\mathbf{d}}\left(-\beta N, \frac{P}{q} \mathbf{l}\right)+\mathcal{O}\left(P^{-A}\right),
\end{aligned}
$$

where the sets $\mathcal{N}_{\mathbf{d}, q}(H)$ and $\mathcal{N}_{\mathbf{d}, q}$ are defined by (60) and (61), respectively.

Some care is needed with the modulus of the congruence in the definition of $\mathcal{N}_{\mathbf{d}, q}(H)$. According to (83) we have $d_{i} \leq D<R^{1 / 6}<p, p^{\prime}$. Thus, since $\delta \mid\left(p p^{\prime}\right)^{2}$, we have

$$
\left(q p p^{\prime} \delta^{-1},\left(a p p^{\prime}+s q\right) \delta^{-1} d_{i}^{2}\right)=\left(q p p^{\prime} \delta^{-1}, d_{i}^{2}\right)=\left(q, d_{i}^{2}\right) .
$$

It follows that the modulus of the congruence occuring in the definition of $\mathcal{N}_{\mathbf{d}, q}(H)$ may be taken to be $\left(q, d_{i}^{2}\right)$ instead of $\left(q p p^{\prime} \delta^{-1}, d_{i}^{2}\right)$.

We use (85), (95) and take into account the expression (96) for the integrand. In this way we find a formula for $\mathcal{E}_{1}^{(1)}$. Then we change the variable $\beta N=\beta^{\prime}$ 
and use (62) to obtain

$$
\begin{aligned}
\mathcal{E}_{1}^{(1)}=P^{4} & \sum_{(\mathcal{R})} \frac{\gamma\left(p p^{\prime}\right)}{\left(p p^{\prime}\right)^{4}} \sum_{(\mathcal{D})} \sum_{\delta \mid\left(p p^{\prime}\right)^{2}} \sum_{q \leq P} \frac{\delta^{3}}{d_{1} d_{2} d_{3} q^{6}} \\
& \times \sum_{\mathbf{n} \in \mathcal{N}_{\mathbf{d}, q}\left(R^{2} \delta^{-1}\right)} \sum_{\mathbf{l} \in \mathcal{N}_{\mathbf{d}, q}} \sum_{s\left(p p^{\prime}\right)^{*}}\left(\frac{s}{p p^{\prime}}\right) e_{p p^{\prime}}(-s N) \sum_{\substack{a(q)^{*} \\
\left(a p p^{\prime}+s q, q p p^{\prime}\right)=\delta}} \\
& \times \int_{\mathcal{B}^{\prime}(q, a)} S_{\mathbf{d}}\left(\frac{q p p^{\prime}}{\delta}, \frac{a p p^{\prime}+s q}{\delta}, \mathbf{n}\right) S_{\mathbf{d}}(q,-a,-\mathbf{l}) \\
& \times I_{\mathbf{d}}\left(\beta,-\frac{P \delta}{q p p^{\prime}} \mathbf{n}\right) I_{\mathbf{d}}\left(-\beta, \frac{P}{q} \mathbf{l}\right) d \beta+\mathcal{O}\left(P^{-A}\right) .
\end{aligned}
$$

We decompose $\mathcal{E}_{1}^{(1)}$ as follows:

$$
\mathcal{E}_{1}^{(1)}=\mathcal{U}+\mathcal{V}+\mathcal{O}\left(P^{-A}\right)
$$

Here $\mathcal{U}$ is the contribution of the terms with $\mathbf{n}=\mathbf{l}=\mathbf{0}$ and $\mathcal{V}$ is the contribution of the other terms.

Consider $\mathcal{V}$. We change the order of summation over $a$ and integration over $\beta$. Using (62) we conclude that in the new expression for $\mathcal{V}$ the domain of integration is $\left\{|\beta| \leq P q^{-1}\right\}$ and we sum over $a$ satisfying the previous conditions and such that $\bar{a}$ belongs to a set $\mathcal{A}(q, \beta)$ of residues $(\bmod q)$. There is no restriction on $\bar{a}$ if $|\beta| \leq P(2 q)^{-1}$. For the other $\beta$ the set $\mathcal{A}(q, \beta)$ is not necessarily a complete set of residues modulo $q$, but we have

$$
\sum_{\substack{a(q)^{*} \\\left(a p p^{\prime}+s q, q p^{\prime}\right)=\delta \\ \bar{a} \in \mathcal{A}(q, \beta)}} \ldots=\sum_{-q / 2<v \leq q / 2} \sigma(v, q, \beta) \sum_{\substack{a(q)^{*} \\\left(a p p^{\prime}+s q, q p p^{\prime}\right)=\delta}} e_{q}(\bar{a} v) \ldots
$$

where the function $\sigma$ satisfies

$$
|\sigma(v, q, \beta)| \ll(1+|v|)^{-1} .
$$

Detailed explanation of this technique can be found in Section 3 of [9], for example.

Lemma $10(i)$ and (65) imply that if $(\mathbf{n}, \mathbf{l}) \neq(\mathbf{0}, \mathbf{0})$ then

$$
\int_{-\infty}^{\infty}\left|I_{\mathbf{d}}\left(\beta,-\frac{P \delta}{q p p^{\prime}} \mathbf{n}\right) I_{\mathbf{d}}\left(-\beta, \frac{P}{q} \mathbf{l}\right)\right| d \beta \ll \frac{q^{2} P^{2 \varepsilon-2}}{\lambda\left(\mathbf{d}, \mathbf{n}, \mathbf{l}, R^{2} \delta^{-1}\right)} .
$$


We use (98) - (100) and Lemma $6(i)$ to get

$$
\mathcal{V}=\mathcal{O}\left(\mathcal{V}^{*}\right)
$$

where

$$
\begin{aligned}
\mathcal{V}^{*}=P^{2+2 \varepsilon} R^{-7} \sum_{(\mathcal{R})} \sum_{(\mathcal{D})} \sum_{\delta \mid\left(p p^{\prime}\right)^{2}} \sum_{q \leq P} \frac{\delta^{3}}{d_{1} d_{2} d_{3} q^{4}} \\
\times \sum_{\mathbf{n} \in \mathcal{N}_{\mathbf{d}, q}\left(R^{2} \delta^{-1}\right)} \sum_{\mathbf{l} \in \mathcal{N}_{\mathbf{d}, q}} \sum_{|v| \leq P} \frac{\left|W\left(\mathbf{d}, \mathbf{n}, \mathbf{l}, p, p^{\prime}, q, v, \delta\right)\right|}{(1+|v|) \lambda\left(\mathbf{d}, \mathbf{n}, \mathbf{l}, R^{2} \delta^{-1}\right)}
\end{aligned}
$$

and

$$
\begin{aligned}
& W\left(\mathbf{d}, \mathbf{n}, \mathbf{l}, p, p^{\prime}, q, v, \delta\right)=\sum_{s\left(p p^{\prime}\right)^{*}}\left(\frac{s}{p p^{\prime}}\right) e_{p p^{\prime}}(-s N) \\
& \times \sum_{\substack{a(q)^{*} \\
\left(a p p^{\prime}+s q, q p p^{\prime}\right)=\delta}} e_{q}(\bar{a} v) S_{\mathbf{d}}\left(\frac{q p p^{\prime}}{\delta}, \frac{a p p^{\prime}+s q}{\delta}, \mathbf{n}\right) S_{\mathbf{d}}(q,-a,-\mathbf{l}) .
\end{aligned}
$$

Consider $\mathcal{U}$. We represent it as

$$
\mathcal{U}=\mathcal{U}^{\prime}+\mathcal{U}^{\prime \prime},
$$

where in $\mathcal{U}^{\prime}$ the integration is taken over the subset $\left\{|\beta| \leq P(2 q)^{-1}\right\}$ of $\mathcal{B}^{\prime}(q, a)$ and, respectively, in $\mathcal{U}^{\prime \prime}$ we integrate over $\mathcal{B}^{\prime}(q, a) \cap\left\{|\beta|>P(2 q)^{-1}\right\}$.

Consider first $\mathcal{U}^{\prime \prime}$. We change the order of summation over $a$ and integration and proceed as in the treatment of $\mathcal{V}$. We apply the simple estimate

$$
\int_{|\beta|>P(2 q)^{-1}}|I(\beta)|^{6} d \beta \ll q^{2} P^{-2}
$$

and use (65) to establish that

$$
\mathcal{U}^{\prime \prime}=\mathcal{O}\left(\mathcal{V}^{*}\right)
$$

Consider now $\mathcal{U}^{\prime}$. In this case the set of integration does not depend on $a$. We extend the domain of integration to the real line. Taking into account (23), (65) and (105) we find that

$$
\mathcal{U}=\mathcal{U}_{1}+\mathcal{O}\left(\mathcal{V}^{*}\right)
$$

where

$$
\mathcal{U}_{1}=\kappa_{0} P^{4} \sum_{(\mathcal{R})} \frac{\gamma\left(p p^{\prime}\right)}{\left(p p^{\prime}\right)^{4}} \sum_{(\mathcal{D})} \sum_{\delta \mid\left(p p^{\prime}\right)^{2}} \sum_{q \leq P} \frac{\delta^{3} W\left(\mathbf{d}, \mathbf{0}, \mathbf{0}, p, p^{\prime}, q, 0, \delta\right)}{d_{1} d_{2} d_{3} q^{6}} .
$$

From (97), (101), (104), (106) and (107) we obtain

$$
\mathcal{E}_{1}^{(1)}=\mathcal{U}_{1}+\mathcal{O}\left(\mathcal{V}^{*}\right) .
$$




\subsubsection{The sum $\mathcal{E}_{1}^{(2)}$.}

In this section we shall establish the asymptotic formula (111). The main term in it coincides with the main term in (109). The remainder terms are complicated expressions and we shall study them later.

Consider the integral $J_{1}^{(2)}$, defined by (87). We decompose again the unit interval as in the previous section and find that

$$
\begin{aligned}
J_{1}^{(2)}= & \sum_{\delta \mid\left(p p^{\prime}\right)^{2}} \sum_{q \leq P} \\
& \times \sum_{\substack{a(q)^{*} \\
\left(a p p^{\prime}+s q, q p p^{\prime}\right)=\delta}} \int_{\mathcal{B}(q, a)} f_{\mathbf{d}}\left(\frac{a}{q}+\frac{s}{p p^{\prime}}+\beta\right) \mathcal{W}_{\mathbf{d}, Q}\left(-\frac{a}{q}-\beta\right) d \alpha .
\end{aligned}
$$

To deal with $f_{\mathbf{d}}$ and $\mathcal{W}_{\mathbf{d}, Q}$ we use Lemmas 12 and 16 , respectively. We conclude that if $q \leq Q$ then the integrand in the right hand side of (110) equals

$$
\begin{gathered}
\frac{P^{6} \delta^{3}}{q^{6}\left(p p^{\prime}\right)^{3}\left(d_{1} d_{2} d_{3}\right)^{2}} \sum_{\mathbf{n} \in \mathcal{N}_{\mathbf{d}, q}\left(R^{2} \delta^{-1}\right)} S_{\mathbf{d}}\left(\frac{q p p^{\prime}}{\delta}, \frac{a p p^{\prime}+s q}{\delta}, \mathbf{n}\right) S_{\mathbf{d}}(q,-a) \\
\times I_{\mathbf{d}}\left(\beta N,-\frac{P \delta}{q p p^{\prime}} \mathbf{n}\right) I^{3}(-\beta N)+\mathcal{O}\left(P^{-A}\right) .
\end{gathered}
$$

If $Q<q \leq P$ then the integrand is $\mathcal{O}\left(P^{-A}\right)$. From this observation, (85) and (110) we find an expression for $\mathcal{E}_{1}^{(2)}$. We change the variable in the integral and obtain

$$
\begin{aligned}
\mathcal{E}_{1}^{(2)}=P^{4} & \sum_{(\mathcal{R})} \frac{\gamma\left(p p^{\prime}\right)}{\left(p p^{\prime}\right)^{4}} \sum_{(\mathcal{D})} \sum_{\delta \mid\left(p p^{\prime}\right)^{2}} \sum_{q \leq Q} \frac{\delta^{3}}{d_{1} d_{2} d_{3} q^{6}} \\
& \times \sum_{\mathbf{n} \in \mathcal{N}_{\mathbf{d}, q}\left(R^{2} \delta^{-1}\right)} \sum_{s\left(p p^{\prime}\right)^{*}}\left(\frac{s}{p p^{\prime}}\right) e_{p p^{\prime}}(-s N) \sum_{\substack{a(q)^{*} \\
\left(a p p^{\prime}+s q, q p p^{\prime}\right)=\delta}} \\
& \times \int_{\mathcal{B}^{\prime}(q, a)} S_{\mathbf{d}}\left(\frac{q p p^{\prime}}{\delta}, \frac{a p p^{\prime}+s q}{\delta}, \mathbf{n}\right) S_{\mathbf{d}}(q,-a) \\
& \quad \times I_{\mathbf{d}}\left(\beta,-\frac{P \delta}{q p p^{\prime}} \mathbf{n}\right) I^{3}(-\beta) d \beta+\mathcal{O}\left(P^{-A}\right) .
\end{aligned}
$$

Proceeding as in Section 3.4.2 we find that the contribution to $\mathcal{E}_{1}^{(2)}$ from the terms with $\mathbf{n} \neq \mathbf{0}$ is $\mathcal{O}\left(\mathcal{V}^{*}\right)$, where $\mathcal{V}^{*}$ is defined by (102). 
Let $\mathcal{U}^{*}$ be the contribution to $\mathcal{E}_{1}^{(2)}$ from the terms with $\mathbf{n}=\mathbf{0}$. Arguing as in Section 3.4.2 we see that $\mathcal{U}^{*}=\mathcal{U}^{* *}+\mathcal{O}\left(\mathcal{V}^{*}\right)$, where in $\mathcal{U}^{* *}$ the integration is taken over the real line.

Let us compare the expression $\mathcal{U}^{* *}$ with $\mathcal{U}_{1}$, defined by (108). The only difference is that in the first one we sum over $q \leq Q$, whilst in the second the summation is taken over $q \leq P$. Hence we have

$$
\mathcal{E}_{1}^{(2)}=\mathcal{U}_{1}+\mathcal{O}\left(\mathcal{V}^{*}\right)+\mathcal{O}\left(D^{*}\right)
$$

where

$$
\begin{aligned}
D^{*}= & P^{4} R^{-7} \sum_{(\mathcal{R})} \sum_{(\mathcal{D})} \sum_{\delta \mid\left(p p^{\prime}\right)^{2}} \\
& \times \sum_{\min \left(Q, Q \delta\left(p p^{\prime}\right)^{-1}\right)<q \leq P} \frac{\delta^{3}\left|W\left(\mathbf{d}, \mathbf{0}, \mathbf{0}, p, p^{\prime}, q, 0, \delta\right)\right|}{d_{1} d_{2} d_{3} q^{6}},
\end{aligned}
$$

and where $W$ is defined by (103). Here we have defined $D^{*}$ with a longer range of summation for $q$ than is needed at this point. We do this because we shall encounter, in the next section, an error term whose estimation will involve the sum $D^{*}$ as defined above.

\subsubsection{The sum $\mathcal{E}_{1}^{(3)}$.}

The object of this section is to establish the asymptotic formula (121). The main term there coincides with the main terms in (109) and (111). The error terms in (121) are complicated and we shall estimate them in the next sections.

Consider the quantity $J_{1}^{(3)}$, defined by (88). We use (29) to get

$$
\begin{aligned}
J_{1}^{(3)}= & \frac{P^{2}}{\left(d_{1} d_{2} d_{3}\right)^{2}} \sum_{q_{1}, q_{2} \leq Q}\left(q_{1} q_{2}\right)^{-3} \\
& \times \sum_{a_{1}\left(q_{1}\right)^{*}, a_{2}\left(q_{2}\right)^{*}} S_{\mathbf{d}}\left(q_{2},-a_{2}\right) S_{\mathbf{d}}\left(q_{1},-a_{1}\right) B\left(\frac{a_{1}}{q_{1}}+\frac{s}{p p^{\prime}}+\frac{a_{2}}{q_{2}}\right),
\end{aligned}
$$

where

$$
B(\alpha)=\sum_{n \in \mathbb{Z}} H^{2}\left(\frac{n}{N}\right) e(n \alpha)
$$

We apply Poisson's summation formula to obtain

$$
B(\alpha)=P^{2} \sum_{k \in \mathbb{Z}} \int_{-\infty}^{\infty} H^{2}(t) e((\alpha-k) N t) d t .
$$

Now we use Lemma 11 and after some standard calculations we find that

$$
B(\alpha)=\left\{\begin{array}{lll}
\kappa_{0} P^{2}+\mathcal{O}\left(P^{-A}\right) & \text { if } & \alpha \in \mathbb{Z} \\
\mathcal{O}\left(P^{-A}\right) & \text { if } & \|\alpha\| \geq P^{\varepsilon-2}
\end{array}\right.
$$


Using (85) and (113) we find an expression for $\mathcal{E}_{1}^{(3)}$. Then we take into account (115) to obtain

$$
\mathcal{E}_{1}^{(3)}=D+E+\mathcal{O}\left(P^{-A}\right),
$$

where $D$ is the contribution of the terms with $\frac{a_{1}}{q_{1}}+\frac{s}{p p^{\prime}}+\frac{a_{2}}{q_{2}} \in \mathbb{Z}$ and $E$ comes from the terms with $0<\left\|\frac{a_{1}}{q_{1}}+\frac{s}{p p^{\prime}}+\frac{a_{2}}{q_{2}}\right\|<P^{\varepsilon-2}$.

Consider $E$. We have

$$
E=P^{2} \sum_{(\mathcal{R})} \frac{\gamma\left(p p^{\prime}\right)}{p p^{\prime}} \sum_{(\mathcal{D})}\left(d_{1} d_{2} d_{3}\right)^{-1} \sum_{n \in \mathbb{Z}} H^{2}\left(\frac{n}{N}\right) \mathcal{F},
$$

where

$$
\begin{aligned}
\mathcal{F} & =\sum_{\substack{q_{1}, q_{2} \leq Q\\
}}\left(q_{1} q_{2}\right)^{-3} \sum_{\substack{\left.a_{1}\left(q_{1}\right)^{*}, a_{2}\left(q_{2}\right)^{*} \\
0<\| p^{\prime}\right)^{*}}}\left(\frac{s}{p p^{\prime}}\right) e_{p p^{\prime}}(-s N) \\
& S_{\mathbf{d}}\left(q_{1},-a_{1}\right) S_{\mathbf{d}}\left(q_{2},-a_{2}\right) e\left(n \left(\frac{a_{1}}{q_{1}}+\frac{s}{q_{1}}+\frac{a_{2}}{q_{2}} \|<P^{\varepsilon-2}\right.\right. \\
& \left.\left.+\frac{a_{2}}{q_{2}}\right)\right) .
\end{aligned}
$$

Therefore we have

$$
E=\mathcal{O}\left(E^{*}\right),
$$

where

$$
E^{*}=P^{2} R^{-1} \sum_{(\mathcal{R})} \sum_{(\mathcal{D})}\left(d_{1} d_{2} d_{3}\right)^{-1} \sum_{0<n<N}|\mathcal{F}|
$$

Consider $D$. If we denote $\left(a_{1} p p^{\prime}+s q_{1}, q_{1} p p^{\prime}\right)=\delta$ then it is easy to see that $\delta \mid\left(p p^{\prime}\right)^{2}$. The condition $\frac{a_{1}}{q_{1}}+\frac{s}{p p^{\prime}}+\frac{a_{2}}{q_{2}} \in \mathbb{Z}$ implies that $q_{2}=q_{1} p p^{\prime} \delta^{-1}$ and $a_{2} \equiv-\left(a_{1} p p^{\prime}+s q_{1}\right) \delta^{-1}\left(q_{2}\right)$ and consequently

$$
S_{\mathbf{d}}\left(q_{2},-a_{2}\right)=S_{\mathbf{d}}\left(\frac{q_{1} p p^{\prime}}{\delta}, \frac{a_{1} p p^{\prime}+s q_{1}}{\delta}\right) .
$$

We use this observation and (115) to get

$$
\begin{aligned}
D=\kappa_{0} P^{4} & \sum_{(\mathcal{R})} \frac{\gamma\left(p p^{\prime}\right)}{\left(p p^{\prime}\right)^{4}} \sum_{(\mathcal{D})} \sum_{\delta \mid\left(p p^{\prime}\right)^{2}} \\
& \times \sum_{q \leq \min \left(Q, Q \delta\left(p p^{\prime}\right)^{-1}\right)} \frac{\delta^{3} W\left(\mathbf{d}, \mathbf{0}, \mathbf{0}, p, p^{\prime}, q, 0, \delta\right)}{d_{1} d_{2} d_{3} q^{6}}+\mathcal{O}\left(P^{-A}\right) .
\end{aligned}
$$


It is now clear that

$$
D=\mathcal{U}_{1}+\mathcal{O}\left(D^{*}\right)
$$

where $\mathcal{U}_{1}$ and $D^{*}$ are defined by (108) and (112), respectively.

From (116), (118) and (120) we obtain

$$
\mathcal{E}_{1}^{(3)}=\mathcal{U}_{1}+\mathcal{O}\left(D^{*}\right)+\mathcal{O}\left(E^{*}\right)
$$

\subsubsection{The estimation of $\mathcal{V}^{*}$ and $D^{*}$.}

We use definitions (102) and (112) of $\mathcal{V}^{*}$ and $D^{*}$, respectively, to get

$$
\begin{aligned}
& \mathcal{V}^{*} \ll \mathcal{V}_{1}+\mathcal{V}_{p}+\mathcal{V}_{p^{2}}+\mathcal{V}_{p p^{\prime}}+\mathcal{V}_{p^{2} p^{\prime}}+\mathcal{V}_{p^{2} p^{\prime 2}}, \\
& D^{*} \ll D_{1}+D_{p}+D_{p^{2}}+D_{p p^{\prime}}+D_{p^{2} p^{\prime}}+D_{p^{2} p^{\prime 2}},
\end{aligned}
$$

where $\mathcal{V}_{\delta}$ and $D_{\delta}$ are the contributions coming from the corresponding values of $\delta$.

The estimations of $\mathcal{V}_{1}$ and $\mathcal{V}_{p}$ are the most difficult because the domain of summation over $\mathbf{n}$ is largest in these cases. The other terms from the right hand sides of (122) and (123) are much simpler.

Two more Lemmas. Consider the function

$$
\Theta(\mathbf{d}, \mathbf{n}, \mathbf{l}, q, h, v)=\sum_{a(q)^{*}} e_{q}(\bar{a} v) S_{\mathbf{d}}\left(q, a h^{2}, \mathbf{n}\right) S_{\mathbf{d}}(q,-a,-\mathbf{l}) .
$$

To emphasize the dependence of $q$ we will write also $\Theta(q)$ for simplicity.

Define

$$
\eta=\eta(\mathbf{d}, \mathbf{n}, \mathbf{l}, h, v)=\left(2 h d_{1} d_{2} d_{3}\right)^{2} v+\sum_{i=1}^{3} \frac{\left(d_{1} d_{2} d_{3}\right)^{2}}{d_{i}^{2}}\left(h^{2} l_{i}^{2}-n_{i}^{2}\right) .
$$

The following Lemma holds:

Lemma 17. The function $\Theta$ is multiplicative with respect to $q$. Suppose that $d_{1}, d_{2}, d_{3}$ are squarefree odd numbers. If $(h, 2)=1$ then $\Theta\left(2^{\alpha}\right) \ll\left(2^{\alpha}\right)^{4}$. If $(q, 2 h)=1$ then

$$
|\Theta(q)| \leq q^{3}(q \xi(q, \mathbf{d}), \eta(\mathbf{d}, \mathbf{n}, \mathbf{l}, h, v)) .
$$

Proof: First we note that if $\left(q_{1}, q_{2}\right)=\left(a_{1}, q_{1}\right)=\left(a_{2}, q_{2}\right)=1$ then the following simple identity holds:

$$
e_{q_{1} q_{2}}\left(\overline{\left(a_{1} q_{2}+a_{2} q_{1}\right)} m\right)=e_{q_{1}}\left(\overline{\left(a_{1} q_{2}^{2}\right)} m\right) e_{q_{2}}\left(\overline{\left(a_{2} q_{1}^{2}\right)} m\right) .
$$


The multiplicativity of $\Theta$ with respect to $q$ is an easy consequence of (127) and Lemma $2(i)$. If $(h, 2)=1$ then the estimate $\Theta\left(2^{\alpha}\right) \ll\left(2^{\alpha}\right)^{4}$ follows from Lemma 2 (iii).

It remains to prove $(126)$ provided that $(q, 2 h)=1$. It is enough to establish that for any prime $p \nmid 2 h$ and for any integer $k$ we have

$$
\left|\Theta\left(p^{k}\right)\right| \leq p^{3 k}\left(p^{k} \xi\left(p^{k}, \mathbf{d}\right), \eta\right) .
$$

We may suppose that $n_{i}, l_{i} \equiv 0\left(\left(p^{k}, d_{i}^{2}\right)\right)$ because otherwise, according to Lemma 2 (ii), we have $\Theta=0$ and (128) is true.

Let $d_{i}=p^{\mu_{i}} e_{i}$, where $p \nmid e_{i}$. Then $\mu_{i}=0$ or $\mu_{i}=1$ and $\left(p^{k}, d_{i}^{2}\right)=$ $p^{\nu_{i}}$, where $\nu_{i}=\min \left(k, 2 \mu_{i}\right)$. Let $n_{i}^{\prime}=n_{i} p^{-\nu_{i}}$ and $l_{i}^{\prime}=l_{i} p^{-\nu_{i}}$. We have by Lemma 2 (ii) and $(i v)$

$$
\Theta\left(p^{k}\right)=\prod_{i=1}^{3}\left(\left(\frac{-1}{p^{k-\nu_{i}}}\right) p^{2 \nu_{i}} S^{2}\left(p^{k-\nu_{i}}, 1\right)\right) \rho\left(p^{k}\right),
$$

where

$$
\rho\left(p^{k}\right)=\sum_{a\left(p^{k}\right)^{*}} e_{p^{k}}(\bar{a} v) \prod_{\substack{i=1 \\ \nu_{i}<k}}^{3} e_{p^{k-\nu_{i}}}\left({\overline{\left(4 a e_{i}^{2}\right)}}_{p^{k-\nu_{i}}}\left(l_{i}^{\prime 2}-{\overline{\left(h^{2}\right)}}_{p^{k-\nu_{i}}} n_{i}^{\prime 2}\right)\right) .
$$

Applying Lemma 2 (iii) we get

$$
\left|\Theta\left(p^{k}\right)\right| \leq p^{3 k+\nu_{1}+\nu_{2}+\nu_{3}}\left|\rho\left(p^{k}\right)\right| .
$$

Consider $\rho\left(p^{k}\right)$. It is easy to see that if $p \nmid A$ then $e_{p^{k}}\left(\overline{(A)}_{p^{k}} p^{\nu}\right)=$ $e_{p^{k-\nu}}\left(\overline{(A)}_{p^{k-\nu}}\right)$. Hence

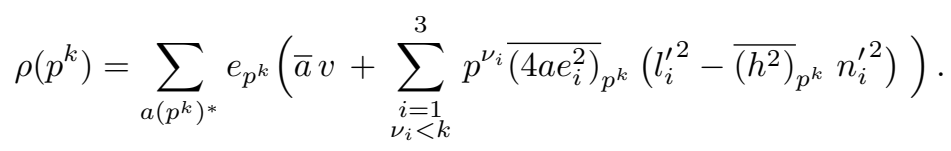

We can already drop the condition $\nu_{i}<k$ from the domain of summation of the inner sum because the terms with $\nu_{i}=k$ do not contribute to $\rho\left(p^{k}\right)$. We obtain

$$
\rho\left(p^{k}\right)=\sum_{a\left(p^{k}\right)^{*}} e_{p^{k}}\left(\overline{\left(a\left(2 h e_{1} e_{2} e_{3}\right)^{2}\right)} \eta^{\prime}\right)=c_{p^{k}}\left(\eta^{\prime}\right),
$$

where $c_{q}(n)$ is the Ramanujan sum, defined by (4), and

$$
\eta^{\prime}=\left(2 h e_{1} e_{2} e_{3}\right)^{2} v+\sum_{i=1}^{3} p^{\nu_{i}}\left(e_{1} e_{2} e_{3}\right)^{2} e_{i}^{-2}\left(h^{2}{l_{i}^{\prime}}^{2}-{n_{i}^{\prime}}^{2}\right) .
$$

Lemma 5 gives

$$
\left|\rho\left(p^{k}\right)\right| \leq\left(p^{k}, \eta^{\prime}\right)
$$


Therefore using (129) and (130) we get

$$
\left|\Theta\left(p^{k}\right)\right| \leq p^{3 k+\nu_{1}+\nu_{2}+\nu_{3}}\left(p^{k}, \eta^{\prime}\right)=p^{3 k}\left(p^{k+\nu_{1}+\nu_{2}+\nu_{3}}, \eta^{\prime \prime}\right),
$$

where $\eta^{\prime \prime}=p^{\nu_{1}+\nu_{2}+\nu_{3}} \eta^{\prime}$.

If $k \geq 2$ then $\nu_{i}=2 \mu_{i}$ and $\eta^{\prime \prime}=\eta$, so the inequality (128) holds.

Consider the case $k=1$. Now $\nu_{i}=\mu_{i}$ and we have

$$
|\Theta(p)| \leq p^{3}\left(p^{1+\mu_{1}+\mu_{2}+\mu_{3}}, \eta^{\prime \prime}\right) \leq p^{3}\left(p^{1+\mu_{1}+\mu_{2}+\mu_{3}}, \eta^{\prime \prime \prime}\right)
$$

where

$$
\eta^{\prime \prime \prime}=p^{\mu_{1}+\mu_{2}+\mu_{3}} \eta^{\prime \prime}=\left(2 h d_{1} d_{2} d_{3}\right)^{2} v+\sum_{i=1}^{3} p^{\mu_{i}}\left(d_{1} d_{2} d_{3}\right)^{2} d_{i}^{-2}\left(h^{2} l_{i}^{2}-n_{i}^{2}\right) .
$$

It is easy to see that $p^{1+\mu_{1}+\mu_{2}+\mu_{3}} \mid\left(\eta^{\prime \prime \prime}-\eta\right)$, whence

$$
\left(p^{1+\mu_{1}+\mu_{2}+\mu_{3}}, \eta^{\prime \prime \prime}\right)=\left(p^{1+\mu_{1}+\mu_{2}+\mu_{3}}, \eta\right) .
$$

Using (131) and (132) we conclude that (128) is true for $k=1$ as well. The Lemma is proved.

Lemma 18. Suppose that $H$ is an integer such that $R \leq H \leq 4 R^{2}$ and let

$$
\begin{aligned}
\mathcal{D}(H)= & \sum_{d_{1}, d_{2}, d_{3} \leq D} \sum_{q \leq P H R^{-2}} \sum_{|v| \leq P} \\
& \times \sum_{\mathbf{n} \in \mathcal{N}_{\mathbf{d}, q}(H)} \sum_{\mathbf{l} \in \mathcal{N}_{\mathbf{d}, q}} \frac{(q \xi(q, \mathbf{d}), \eta(\mathbf{d}, \mathbf{n}, \mathbf{l}, H, v))}{q d_{1} d_{2} d_{3}(1+|v|) \lambda(\mathbf{d}, \mathbf{n}, \mathbf{l}, H)},
\end{aligned}
$$

The following estimate holds:

$$
\mathcal{D}(H) \ll\left(P H^{2} R^{-2}+H^{3}\right) D^{6} P^{10 \varepsilon},
$$

Proof: We have

$$
\mathcal{D}(H) \ll \mathcal{D}^{\prime}+\mathcal{D}^{\prime \prime},
$$

where $\mathcal{D}^{\prime}$ is the contribution of the terms for which $\eta(\mathbf{d}, \mathbf{n}, \mathbf{l}, H, v)=0$, and $\mathcal{D}^{\prime \prime}$ is the contribution of the other terms.

Consider $\mathcal{D}^{\prime \prime}$. In this case the quantity $\eta^{*}=\xi(q, \mathbf{d})^{-1} \eta$ is a non-zero integer because $\mathbf{n}$ and $\mathbf{l}$ satisfy the congruence conditions imposed in the definitions (60) and (61) of $\mathcal{N}_{\mathbf{d}, q}(H)$ and $\mathcal{N}_{\mathbf{d}, q}$, respectively. It is easy to see that 
$\sum_{q \leq P H R^{-2}}\left(q, \eta^{*}\right) q^{-1} \ll P^{\varepsilon}$. Hence, using (69), we get

$$
\begin{aligned}
(136) \mathcal{D}^{\prime \prime} & \ll \sum_{d_{1}, d_{2}, d_{3} \leq D} \sum_{\substack{h_{i} \mid d_{i}^{2} \\
i=1,2,3}} \frac{h_{1} h_{2} h_{3}}{d_{1} d_{2} d_{3}} \\
& \times \sum_{\mathbf{n} \in \mathcal{N}^{*}} \sum_{\mathbf{1} \in \mathcal{N}_{\mathbf{d}, 1}} \lambda(\mathbf{d}, \mathbf{n}, \mathbf{l}, H)^{-1} \sum_{\substack{|v| \leq P \\
\eta \neq 0}} \frac{1}{1+|v|} \sum_{\substack{q \leq P H R^{-2} \\
\left(q, d_{i}^{2}\right)=h_{i} \\
i=1,2,3}} \frac{\left(q, \eta^{*}\right)}{q} \\
& \ll P^{2 \varepsilon} \mathcal{H},
\end{aligned}
$$

where

$$
\mathcal{H}=\sum_{d_{1}, d_{2}, d_{3} \leq D} \sum_{\substack{h_{i} \mid d_{i}^{2} \\ i=1,2,3}} \frac{h_{1} h_{2} h_{3}}{d_{1} d_{2} d_{3}} \sum_{\mathbf{n} \in \mathcal{N}^{*}} \sum_{\mathbf{l} \in \mathcal{N}_{\mathbf{d}, 1}} \lambda(\mathbf{d}, \mathbf{n}, \mathbf{l}, H)^{-1}
$$

and

$$
\mathcal{N}^{*}=\left\{\mathbf{n} \in \mathbb{Z}^{3}:\left|n_{i}\right| \leq 4 d_{i} H P^{\varepsilon}, n_{i} \equiv 0\left(h_{i}\right), i=1,2,3\right\} .
$$

We have

$$
\mathcal{H} \ll \mathcal{H}^{\prime}+\mathcal{H}^{\prime \prime}
$$

where $\mathcal{H}^{\prime}$ is the contribution of the terms with $\mathbf{l}=\mathbf{0}$ and $\mathcal{H}^{\prime \prime}$ is the contribution of the other terms.

Consider $\mathcal{H}^{\prime \prime}$. According to (65) and Lemma 13 we have

$$
\sum_{\substack{\mathbf{l} \in \mathcal{N}_{\mathbf{d}, 1} \\ \mathbf{l} \neq \mathbf{0}}} \lambda(\mathbf{d}, \mathbf{n}, \mathbf{l}, H)^{-1} \ll \sum_{\mathbf{l} \in \mathcal{N}_{\mathbf{d}, 1}} \lambda(\mathbf{d}, \mathbf{0}, \mathbf{l})^{-1} \ll D^{3} P^{\varepsilon} .
$$

Our assumption (83) implies that $D \leq R^{1 / 6}$ and we also have $R \leq H$. Thus $h_{i} \leq d_{i}^{2} \leq d_{i} D \leq d_{i} H$, and we easily get

$$
\sum_{\mathbf{n} \in \mathcal{N}^{*}} 1 \ll H^{3} P^{3 \varepsilon} \frac{d_{1} d_{2} d_{3}}{h_{1} h_{2} h_{3}} .
$$

Therefore

$$
\mathcal{H}^{\prime \prime} \ll H^{3} D^{6} P^{7 \varepsilon}
$$

Consider $\mathcal{H}^{\prime}$. First we estimate the quantity

$$
\mathcal{B}=\sum_{\mathbf{n} \in \mathcal{N}^{*}} \lambda(\mathbf{d}, \mathbf{n}, \mathbf{0}, H)^{-1} .
$$

We have

$$
\mathcal{B} \ll 1+\mathcal{B}_{1}+\mathcal{B}_{2}+\mathcal{B}_{3},
$$


where $\mathcal{B}_{\nu}$ comes from the terms with $\left|n_{\nu}\right| d_{\nu}^{-1}=\max _{i=1,2,3}\left(\left|n_{i}\right| d_{i}^{-1}\right)>0$. Consider, for example, $\mathcal{B}_{1}$. Using again the inequalities $D \leq R^{1 / 6}$ and $R \leq H$ we obtain

$$
\mathcal{B}_{1} \ll H^{2} \sum_{\substack{1 \leq n_{1} \leq 4 d_{1} H P^{\varepsilon} \\ n_{1} \equiv 0\left(h_{1}\right)}} \frac{d_{1}^{2}}{n_{1}^{2}}\left(1+\frac{d_{2} n_{1}}{h_{2} d_{1}}\right)\left(1+\frac{d_{3} n_{1}}{h_{3} d_{1}}\right) \ll H^{3} P^{\varepsilon} \frac{d_{1} d_{2} d_{3}}{h_{1} h_{2} h_{3}} .
$$

Obviously, the same estimate holds for $\mathcal{B}$ as well. Therefore

$$
\mathcal{H}^{\prime}=\sum_{d_{1}, d_{2}, d_{3} \leq D} \sum_{\substack{h_{i} \mid d_{i}^{2} \\ i=1,2,3}} \frac{h_{1} h_{2} h_{3}}{d_{1} d_{2} d_{3}} \mathcal{B} \ll H^{3} D^{3} P^{2 \varepsilon} .
$$

The estimates (136) - (139) imply

$$
\mathcal{D}^{\prime \prime} \ll H^{3} D^{6} P^{9 \varepsilon} .
$$

Now consider $\mathcal{D}^{\prime}$. We have

$$
\mathcal{D}^{\prime} \ll \sum_{d_{1}, d_{2}, d_{3} \leq D} \sum_{q \leq P H R^{-2}} \frac{\xi(q, \mathbf{d})}{d_{1} d_{2} d_{3}} \mathcal{X},
$$

where

$$
\mathcal{X}=\sum_{|v| \leq P} \sum_{\mathbf{l} \in \mathcal{N}_{\mathbf{d}, q}} \sum_{\substack{\mathbf{n} \in \mathcal{N}_{\mathbf{d}, q}(H) \\ \eta=0}}((1+|v|) \lambda(\mathbf{d}, \mathbf{n}, \mathbf{l}, H))^{-1} .
$$

We divide $\mathcal{X}$ into two parts:

$$
\mathcal{X}=\mathcal{X}^{\prime}+\mathcal{X}^{\prime \prime},
$$

where $\mathcal{X}^{\prime}$ is the contribution from the terms with $\mathbf{l}=\mathbf{0}$ and $\mathcal{X}^{\prime \prime}$ comes from the other terms.

Consider $\mathcal{X}^{\prime \prime}$. Using (65) we get

$$
\mathcal{X}^{\prime \prime} \ll \sum_{\mathbf{l} \in \mathcal{N}_{\mathbf{d}, q}} \lambda(\mathbf{d}, \mathbf{0}, \mathbf{l})^{-1} \sum_{|v| \leq P} \frac{\mathcal{K}(\mathbf{d}, \mathbf{l}, q, H, v)}{(1+|v|)}
$$

where $\mathcal{K}$ is the number of $\mathbf{n} \in \mathcal{N}_{\mathbf{d}, q}(H)$ such that $\eta(\mathbf{d}, \mathbf{n}, \mathbf{l}, H, v)=0$.

Let us estimate $\mathcal{K}$. There are $\mathcal{O}\left(H P^{\varepsilon} d_{1}\left(q, d_{1}^{2}\right)^{-1}\right)$ admissible values of $n_{1}$. If $\mathbf{d}, \mathbf{l}, H, v, n_{1}$ are fixed then the equation $\eta=0$ determines at most $\mathcal{O}\left(P^{\varepsilon}\right)$ pairs $n_{2}, n_{3}$. Therefore

$$
\mathcal{K}(\mathbf{d}, \mathbf{l}, q, H, v) \ll H P^{2 \varepsilon} d_{1}\left(q, d_{1}^{2}\right)^{-1}
$$

and, according to Lemma 13,

$$
\mathcal{X}^{\prime \prime} \ll P^{4 \varepsilon} D^{3} H d_{1}\left(q, d_{1}^{2}\right)^{-1} .
$$


Consider $\mathcal{X}^{\prime}$. The contribution to $\mathcal{X}^{\prime}$ coming from $\mathbf{n}=\mathbf{0}$ is $\mathcal{O}\left(P^{\varepsilon}\right)$. Using (65) and (125) we conclude that if $\mathbf{n} \neq \mathbf{0}$ and $\eta(\mathbf{d}, \mathbf{n}, \mathbf{0}, H, v)=0$ then we have $v>0$ and $\lambda(\mathbf{d}, \mathbf{n}, \mathbf{0}, H)^{-1} \ll v^{-1}$. Therefore

$$
\mathcal{X}^{\prime} \ll P^{\varepsilon}+\sum_{1 \leq v \leq P} v^{-2} \mathcal{K}(\mathbf{d}, \mathbf{0}, q, H, v) \ll H P^{2 \varepsilon} d_{1}\left(q, d_{1}^{2}\right)^{-1} .
$$

From (141) - (144) and Lemma 14 we obtain

$$
\mathcal{D}^{\prime} \ll P^{1+6 \varepsilon} H^{2} D^{6} R^{-2} .
$$

The estimate (134) is a consequence of (135), (140) and (145).

This completes the proof of the Lemma.

Consider $\mathcal{V}_{1}$ and $D_{1}$. We use (102), (112) and the definitions of $\mathcal{V}_{1}$ and $D_{1}$, given at the beginning of this section. We note that if $(a, q)=\left(s, p p^{\prime}\right)=1$ then the conditions $\left(q, p p^{\prime}\right)=1$ and $\left(a p p^{\prime}+s q, q p p^{\prime}\right)=1$ are equivalent. We impose the first of these conditions in the domain of summation over $q$ and omit the second from the domain of summation over $a$. We obtain

$$
\begin{aligned}
\mathcal{V}_{1} \ll P^{2+2 \varepsilon} R^{-7} \sum_{(\mathcal{R})} \sum_{(\mathcal{D})}\left(d_{1} d_{2} d_{3}\right)^{-1} \sum_{\substack{q \leq P \\
\left(q, p p^{\prime}\right)=1}} q^{-4} \\
\times \sum_{\mathbf{n} \in \mathcal{N}_{\mathbf{d}, q}\left(R^{2}\right)} \sum_{\mathbf{l} \in \mathcal{N}_{\mathbf{d}, q}} \sum_{|v| \leq P} \frac{\left|W_{1}\left(\mathbf{d}, \mathbf{n}, \mathbf{l}, p, p^{\prime}, q, v\right)\right|}{(1+|v|) \lambda\left(\mathbf{d}, \mathbf{n}, \mathbf{l}, R^{2}\right)}
\end{aligned}
$$

and

$$
D_{1} \ll P^{4} R^{-7} \sum_{(\mathcal{R})} \sum_{(\mathcal{D})} \sum_{\substack{Q\left(p p^{\prime}\right)^{-1}<q \leq P \\\left(q, p p^{\prime}\right)=1}} \frac{\left|W_{1}\left(\mathbf{d}, \mathbf{0}, \mathbf{0}, p, p^{\prime}, q, 0\right)\right|}{q^{6} d_{1} d_{2} d_{3}}
$$

where

$$
\begin{aligned}
& W_{1}\left(\mathbf{d}, \mathbf{n}, \mathbf{l}, p, p^{\prime}, q, v\right)=\sum_{s\left(p p^{\prime}\right)^{*}}\left(\frac{s}{p p^{\prime}}\right) e_{p p^{\prime}}(-s N) \\
& \times \sum_{a(q)^{*}} e_{q}(\bar{a} v) S_{\mathbf{d}}\left(q p p^{\prime}, a p p^{\prime}+s q, \mathbf{n}\right) S_{\mathbf{d}}(q,-a,-\mathbf{l}) .
\end{aligned}
$$

We apply Lemma $2(i)$ and $(i v)$ and find that

$$
\begin{aligned}
& S\left(q p p^{\prime},\left(a p p^{\prime}+s q\right) d_{i}^{2}, n_{i}\right)=S\left(q, a\left(p p^{\prime}\right)^{2} d_{i}^{2}, n_{i}\right) S\left(p p^{\prime}, s q^{2} d_{i}^{2}, n_{i}\right) \\
& =S\left(q, a\left(p p^{\prime}\right)^{2} d_{i}^{2}, n_{i}\right) S\left(p p^{\prime}, 1\right)\left(\frac{s}{p p^{\prime}}\right) e_{p p^{\prime}}\left(-\overline{\left(4 s q^{2} d_{i}^{2}\right)} n_{i}^{2}\right) .
\end{aligned}
$$


Therefore, using (4), (8), (124), (148) and Lemmas 2 (iii) and 4 we find

$$
\begin{aligned}
W_{1}\left(\mathbf{d}, \mathbf{n}, \mathbf{l}, p, p^{\prime}, q, v\right)= & S^{3}\left(p p^{\prime}, 1\right) K\left(p p^{\prime},-N,-\overline{\left(4 q^{2}\right)} \sum_{i=1}^{3} \overline{\left(d_{i}^{2}\right)} n_{i}^{2}\right) \\
& \times \Theta\left(\mathbf{d}, \mathbf{n}, \mathbf{l}, q, p p^{\prime}, v\right) \\
& \ll R^{4}\left|\Theta\left(\mathbf{d}, \mathbf{n}, \mathbf{l}, q, p p^{\prime}, v\right)\right| .
\end{aligned}
$$

We represent each $q \leq P$, satisfying $\left(q, p p^{\prime}\right)=1$, in the form $q=2^{\alpha} t$, where $\left(t, 2 p p^{\prime}\right)=1$. Hence, using Lemma 17 , we get

$$
\Theta\left(\mathbf{d}, \mathbf{n}, \mathbf{l}, q, p p^{\prime}, v\right) \ll\left(2^{\alpha}\right)^{4} t^{3}\left(t \xi(t, \mathbf{d}), \eta\left(\mathbf{d}, \mathbf{n}, \mathbf{l}, p p^{\prime}, v\right)\right),
$$

where $\xi$ and $\eta$ are defined by (69) and (125), respectively.

Obviously $\left(q, d_{i}^{2}\right)=\left(t, d_{i}^{2}\right)$, hence using (60) and (61) we get

$$
\mathcal{N}_{\mathbf{d}, q}\left(R^{2}\right)=\mathcal{N}_{\mathbf{d}, t}\left(R^{2}\right), \quad \mathcal{N}_{\mathbf{d}, q}=\mathcal{N}_{\mathbf{d}, t} .
$$

Formulas (149) - (151) imply

$$
\begin{aligned}
& \sum_{\substack{q \leq P \\
\left(q, p p^{\prime}\right)=1}} \sum_{\mathbf{n} \in \mathcal{N}_{\mathbf{d}, q}\left(R^{2}\right)} \sum_{\mathbf{l} \in \mathcal{N}_{\mathbf{d}, q}} \frac{\left|W_{1}\left(\mathbf{d}, \mathbf{n}, \mathbf{l}, p, p^{\prime}, q, v\right)\right|}{q^{4} \lambda\left(\mathbf{d}, \mathbf{n}, \mathbf{l}, R^{2}\right)} \\
& \ll R^{4} \sum_{2^{\alpha} \leq P} \sum_{\substack{t \leq P 2^{-\alpha} \\
\left(t, 2 p p^{\prime}\right)=1}} \sum_{\mathbf{n} \in \mathcal{N}_{\mathbf{d}, t}\left(R^{2}\right)} \sum_{\mathbf{l} \in \mathcal{N}_{\mathbf{d}, t}} \frac{\left(t \xi(t, \mathbf{d}), \eta\left(\mathbf{d}, \mathbf{n}, \mathbf{l}, p p^{\prime}, v\right)\right)}{t \lambda\left(\mathbf{d}, \mathbf{n}, \mathbf{l}, R^{2}\right)} \\
& \ll R^{4} P^{\varepsilon} \sum_{\substack{q \leq P \\
\left(q, p p^{\prime}\right)=1}} \sum_{\mathbf{n} \in \mathcal{N}_{\mathbf{d}, q}\left(R^{2}\right)} \sum_{\mathbf{l} \in \mathcal{N}_{\mathbf{d}, q}} \frac{\left(q \xi(q, \mathbf{d}), \eta\left(\mathbf{d}, \mathbf{n}, \mathbf{l}, p p^{\prime}, v\right)\right)}{q \lambda\left(\mathbf{d}, \mathbf{n}, \mathbf{l}, R^{2}\right)} .
\end{aligned}
$$

From (133), (146) and (152) we obtain

$$
\mathcal{V}_{1} \ll P^{2+3 \varepsilon} R^{-3} \sum_{(\mathcal{R})} \mathcal{D}\left(p p^{\prime}\right) .
$$

Now we apply Lemma 18 to get

$$
\mathcal{V}_{1} \ll\left(P^{3} R+P^{2} R^{5}\right) D^{6} P^{15 \varepsilon} .
$$

The estimation of $D_{1}$ is much easier. We use (124), (149) and Lemma 2 (ii) and (iii) to get

$$
W_{1}\left(\mathbf{d}, \mathbf{0}, \mathbf{0}, p, p^{\prime}, q, 0\right) \ll R^{4} q^{4} \xi(q, \mathbf{d}) .
$$

We substitute this estimate for $W_{1}$ in (147). Then we apply Lemma 14 and after some calculations we obtain

$$
D_{1} \ll P^{4+5 \varepsilon} Q^{-1} D^{3} R .
$$

Actually, a stronger estimate for $D_{1}$ is available because in this particular case the Kloosterman sum reduces to Ramanujan's sum. This improvement, however, would not have any influence on our final result. 
Consider $\mathcal{V}_{p}$ and $D_{p}$. We use (102), (112) and the definitions of $\mathcal{V}_{p}$ and $D_{p}$. If $\left(a p p^{\prime}+s q, q p p^{\prime}\right)=p$ then $p \mid q$ and $p^{\prime} \nmid q$, so we impose these conditions in the range of summation over $q$. As $p^{\prime} \nmid a p p^{\prime}+s q$ and $\left(a p^{\prime}+s \frac{q}{p}, \frac{q}{p}\right)=1$ we can relax the condition on $a$ to $\left(a p^{\prime}+s \frac{q}{p}, p\right)=1$. We put $q=r p$ and get

$$
\begin{aligned}
\mathcal{V}_{p} \ll P^{2+2 \varepsilon} R^{-8} \sum_{(\mathcal{R})} \sum_{(\mathcal{D})}\left(d_{1} d_{2} d_{3}\right)^{-1} \sum_{\substack{r \leq P R^{-1} \\
\left(r, p^{\prime}\right)=1}} r^{-4} \\
\quad \times \sum_{\mathbf{n} \in \mathcal{N}_{\mathbf{d}, r}(R)} \sum_{\mathbf{l} \in \mathcal{N}_{\mathbf{d}, r}} \sum_{|v| \leq P} \frac{\left|W_{2}\left(\mathbf{d}, \mathbf{n}, \mathbf{l}, p, p^{\prime}, r, v\right)\right|}{(1+|v|) \lambda(\mathbf{d}, \mathbf{n}, \mathbf{l}, R)}
\end{aligned}
$$

and

$$
D_{p} \ll P^{4} R^{-10} \sum_{(\mathcal{R})} \sum_{(\mathcal{D})} \sum_{\substack{Q\left(p p^{\prime}\right)^{-1}<r \leq P R^{-1} \\\left(r, p^{\prime}\right)=1}} \frac{\left|W_{2}\left(\mathbf{d}, \mathbf{0}, \mathbf{0}, p, p^{\prime}, r, 0\right)\right|}{r^{6} d_{1} d_{2} d_{3}}
$$

where

$$
\begin{aligned}
& W_{2}\left(\mathbf{d}, \mathbf{n}, \mathbf{l}, p, p^{\prime}, r, v\right)=\sum_{s\left(p p^{\prime}\right)^{*}}\left(\frac{s}{p p^{\prime}}\right) e_{p p^{\prime}}(-s N) \\
& \times \sum_{\substack{a(r p)^{*} \\
\left(a p^{\prime}+s r, p\right)=1}} e_{r p}(\bar{a} v) S_{\mathbf{d}}\left(r p p^{\prime}, a p^{\prime}+s r, \mathbf{n}\right) S_{\mathbf{d}}(r p,-a,-\mathbf{l}) .
\end{aligned}
$$

We represent each $r \leq P R^{-1}$ satisfying $\left(r, p^{\prime}\right)=1$ in the form

$$
r=r_{0} r_{1} t
$$

where

$$
r_{0}=2^{\alpha}, \quad r_{1}=p^{\beta}, \quad\left(t, 2 p p^{\prime}\right)=1
$$

We note that

$$
\mathcal{N}_{\mathbf{d}, r}(R)=\mathcal{N}_{\mathbf{d}, t}(R), \quad \mathcal{N}_{\mathbf{d}, r}=\mathcal{N}_{\mathbf{d}, t}
$$

It is clear that the set

$$
\left\{c p^{\prime}+c^{\prime} p \mid c(p)^{*}, c^{\prime}\left(p^{\prime}\right)^{*}\right\}
$$

is a reduced system of residues modulo $p p^{\prime}$. Similarly, the set

$$
\left\{a_{0} r_{1} p t+a_{1} r_{0} t+b r_{0} r_{1} p \mid a_{0}\left(r_{0}\right)^{*}, a_{1}\left(r_{1} p\right)^{*}, \quad b(t)^{*}\right\}
$$

is a reduced system of residues modulo $r_{0} r_{1} p t=r p$. 
It is easy to see that if $s=c p^{\prime}+c^{\prime} p$ and $a=a_{0} r_{1} p t+a_{1} r_{0} t+b r_{0} r_{1} p$ then the condition $\left(a p^{\prime}+s r, p\right)=1$ is equivalent to $\left(a_{1}+c r_{1}, p\right)=1$. From these observations, (127) and the elementary properties of the Jacobi symbol we obtain

$$
\begin{aligned}
& W_{2}\left(\mathbf{d}, \mathbf{n}, \mathbf{l}, p, p^{\prime}, r_{0} r_{1} t, v\right)=\left(\frac{p^{\prime}}{p}\right)\left(\frac{p}{p^{\prime}}\right) \\
& \quad \times \sum_{c(p)^{*} c^{\prime}\left(p^{\prime}\right)^{*}}\left(\frac{c}{p}\right)\left(\frac{c^{\prime}}{p^{\prime}}\right) e_{p}(-c N) e_{p^{\prime}}\left(-c^{\prime} N\right) \\
& \quad \times \sum_{a_{0}\left(r_{0}\right)^{*}} \sum_{\substack{a_{1}\left(r_{1} p\right)^{*} \\
\left(a_{1}+c r_{1}, p\right)=1}} \sum_{b(t)^{*}} \\
& \quad \times e_{r_{0}}\left(\overline{a_{0}\left(r_{1} p t\right)^{2}} v\right) e_{r_{1} p}\left(\overline{a_{1}\left(r_{0} t\right)^{2}} v\right) e_{t}\left(\overline{b\left(r_{0} r_{1} p\right)^{2}} v\right) S^{\prime} S^{\prime \prime},
\end{aligned}
$$

where

$$
S^{\prime}=S_{\mathbf{d}}\left(r_{0} r_{1} p t p^{\prime},\left(a_{0} r_{1} p t+a_{1} r_{0} t+b r_{0} r_{1} p\right) p^{\prime}+\left(c p^{\prime}+c^{\prime} p\right) r_{0} r_{1} t, \mathbf{n}\right)
$$

and

$$
S^{\prime \prime}=S_{\mathbf{d}}\left(r_{0} r_{1} p t,-\left(a_{0} r_{1} p t+a_{1} r_{0} t+b r_{0} r_{1} p\right),-\mathbf{l}\right) .
$$

We use (8) and Lemma $2(i)$ to get

$$
\begin{aligned}
S^{\prime} & =S_{\mathbf{d}}\left(p^{\prime}, c^{\prime}\left(r_{0} r_{1} p t\right)^{2}, \mathbf{n}\right) \\
& \times S_{\mathbf{d}}\left(r_{0}, a_{0}\left(r_{1} p t p^{\prime}\right)^{2}, \mathbf{n}\right) \\
& \times S_{\mathbf{d}}\left(r_{1} p,\left(a_{1}+c r_{1}\right)\left(r_{0} t p^{\prime}\right)^{2}, \mathbf{n}\right) \\
& \times S_{\mathbf{d}}\left(t, b\left(r_{0} r_{1} p p^{\prime}\right)^{2}, \mathbf{n}\right)
\end{aligned}
$$

and

$$
\begin{aligned}
S^{\prime \prime}= & S_{\mathbf{d}}\left(r_{0},-a_{0}\left(r_{1} p t\right)^{2},-\mathbf{l}\right) \\
& \times S_{\mathbf{d}}\left(r_{1} p,-a_{1}\left(r_{0} t\right)^{2},-\mathbf{l}\right) \\
& \times S_{\mathbf{d}}\left(t,-b\left(r_{0} r_{1} p\right)^{2},-\mathbf{l}\right) .
\end{aligned}
$$

From (124) and (161) - (163) we get

$$
W_{2}\left(\mathbf{d}, \mathbf{n}, \mathbf{l}, p, p^{\prime}, r_{0} r_{1} t, v\right)=\left(\frac{p^{\prime}}{p}\right)\left(\frac{p}{p^{\prime}}\right) W_{2}^{\prime} W_{2}^{(0)} W_{2}^{(1)} W_{2}^{(2)},
$$


where

$$
\begin{aligned}
W_{2}^{\prime}= & \sum_{c^{\prime}\left(p^{\prime}\right)^{*}}\left(\frac{c^{\prime}}{p^{\prime}}\right) e_{p^{\prime}}\left(-c^{\prime} N\right) S_{\mathbf{d}}\left(p^{\prime}, c^{\prime}\left(r_{0} r_{1} p t\right)^{2}, \mathbf{n}\right), \\
W_{2}^{(0)}= & \Theta\left(\mathbf{d}, \mathbf{n}, \mathbf{l}, r_{0}, p^{\prime}, v\right) . \\
W_{2}^{(1)}= & \sum_{c(p)^{*}}\left(\frac{c}{p}\right) e_{p}(-c N) \sum_{\substack{a_{1}\left(r_{1} p\right)^{*} \\
\left(a_{1}+c r_{1}, p\right)=1}} e_{r_{1} p}\left(\overline{a_{1}\left(r_{0} t\right)^{2}} v\right) \\
& \times S_{\mathbf{d}}\left(r_{1} p,\left(a_{1}+c r_{1}\right)\left(r_{0} t p^{\prime}\right)^{2}, \mathbf{n}\right) S_{\mathbf{d}}\left(r_{1} p,-a_{1}\left(r_{0} t\right)^{2},-\mathbf{l}\right), \\
W_{2}^{(2)}= & \Theta\left(\mathbf{d}, \mathbf{n}, \mathbf{l}, t, p^{\prime}, v\right) .
\end{aligned}
$$

From (4), (8) and Lemmas $2(i i i),(i v)$ and 4 we get

$$
W_{2}^{\prime}=S^{3}\left(p^{\prime}, 1\right) K\left(p^{\prime},-N,-\sum_{i=1}^{3} \overline{4\left(r_{0} r_{1} p t d_{i}\right)^{2}} n_{i}^{2}\right) \ll R^{2} .
$$

Applying only (8) and Lemma 2 (iii) we find that

$$
W_{2}^{(1)} \ll r_{1}^{4} R^{5} .
$$

Finally, Lemma 17 implies

$$
W_{2}^{(0)} \ll r_{0}^{4}
$$

and

$$
W_{2}^{(2)} \ll t^{3}\left(t \xi(t, \mathbf{d}), \eta\left(\mathbf{d}, \mathbf{n}, \mathbf{l}, p^{\prime}, v\right)\right) .
$$


Taking into account (157) - (159) and (164) - (168) we obtain

$$
\begin{aligned}
& \sum_{\substack{r \leq P R^{-1} \\
\left(r, p^{\prime}\right)=1}} \sum_{\mathbf{n} \in \mathcal{N}_{\mathbf{d}, r}(R)} \sum_{\mathbf{l} \in \mathcal{N}_{\mathbf{d}, r}} \frac{\left|W_{2}\left(\mathbf{d}, \mathbf{n}, \mathbf{l}, p, p^{\prime}, r, v\right)\right|}{r^{4} \lambda(\mathbf{d}, \mathbf{n}, \mathbf{l}, R)} \\
& \ll \sum_{2^{\alpha} \leq P} \sum_{p^{\beta} \leq P} \sum_{\substack{t \leq P R^{-1} \\
\left(t, 2 p p^{\prime}\right)=1}} \sum_{\mathbf{n} \in \mathcal{N}_{\mathbf{d}, t}(R)} \sum_{\mathbf{l} \in \mathcal{N}_{\mathbf{d}, t}} \\
& \times \frac{R^{2}\left(2^{\alpha}\right)^{4} R^{5}\left(p^{\beta}\right)^{4} t^{3}\left(t \xi(t, \mathbf{d}), \eta\left(\mathbf{d}, \mathbf{n}, \mathbf{l}, p^{\prime}, v\right)\right)}{\left(2^{\alpha} p^{\beta} t\right)^{4} \lambda(\mathbf{d}, \mathbf{n}, \mathbf{l}, R)} \\
& \ll P^{\varepsilon} R^{7} \sum_{\substack{q \leq P R^{-1} \\
\left(q, p^{\prime}\right)=1}} \sum_{\mathbf{n} \in \mathcal{N}_{\mathbf{d}, q}(R)} \sum_{\substack{\mathbf{l} \in \mathcal{N}_{\mathbf{d}, q}\\
}} \frac{\left(q \xi(q, \mathbf{d}), \eta\left(\mathbf{d}, \mathbf{n}, \mathbf{l}, p^{\prime}, v\right)\right)}{q \lambda(\mathbf{d}, \mathbf{n}, \mathbf{l}, R)}
\end{aligned}
$$

From (155) and (169) we get

$$
\mathcal{V}_{p} \ll P^{2+3 \varepsilon} R^{-1} \sum_{(\mathcal{R})} \mathcal{D}\left(p^{\prime}\right),
$$

where $\mathcal{D}(H)$ is defined by (133). It remains to apply Lemma 18 and we find

$$
\mathcal{V}_{p} \ll\left(P^{3} R+P^{2} R^{4}\right) D^{6} P^{15 \varepsilon} .
$$

The estimation of $D_{p}$ is much simpler. For any $r$ satisfying the conditions imposed in (156) we have

$$
W_{2}\left(\mathbf{d}, \mathbf{0}, \mathbf{0}, p, p^{\prime}, r, 0\right) \ll R^{7} r^{4} \xi(r, \mathbf{d}) .
$$

To prove this inequality we first represent $r$ in the form (157), where $r_{0}, r_{1}, t$ satisfy (158). Then we use formula (164), inequalities (165) - (167) as well as the inequality $W_{2}^{(2)} \ll t^{4} \xi(t, \mathbf{d})$, which is a weak version of (168). The estimate (171) follows.

From (69), (156), (171) and Lemma 14 we obtain

$$
D_{p} \ll P^{4+3 \varepsilon} Q^{-1} D^{3} R .
$$

Here again a sharper estimate is available, but this is of no importance for our result.

Consider $\mathcal{V}_{p^{2}}$ and $D_{p^{2}}$. If $\left(a p p^{\prime}+s q, q p p^{\prime}\right)=p^{2}$ then $p \mid q, p^{2} \nmid q$, $p^{\prime} \nmid q$. We put $q=r p$. The condition $\left(a p p^{\prime}+s q, q p p^{\prime}\right)=p^{2}$ is equivalent to 
$a p^{\prime}+s r \equiv 0(p)$ and, obviously, $\mathcal{N}_{\mathbf{d}, q}=\mathcal{N}_{\mathbf{d}, r}$. We use (102) and (112) to get

$$
\begin{aligned}
\mathcal{V}_{p^{2}} & \ll P^{2+2 \varepsilon} R^{-5} \sum_{(\mathcal{R})} \sum_{(\mathcal{D})}\left(d_{1} d_{2} d_{3}\right)^{-1} \sum_{\substack{r \leq P R^{-1} \\
\left(r, p p^{\prime}\right)=1}} r^{-4} \\
& \times \sum_{\mathbf{n} \in \mathcal{N}_{\mathbf{d}, r}} \sum_{\mathbf{l} \in \mathcal{N}_{\mathbf{d}, r}} \sum_{|v| \leq P} \frac{\left|W_{3}\left(\mathbf{d}, \mathbf{n}, \mathbf{l}, p, p^{\prime}, r, v\right)\right|}{(1+|v|) \lambda(\mathbf{d}, \mathbf{n}, \mathbf{l})}
\end{aligned}
$$

and

$$
D_{p^{2}} \ll P^{4} R^{-7} \sum_{(\mathcal{R})} \sum_{(\mathcal{D})} \sum_{\substack{Q(4 R)^{-1}<r \leq P R^{-1} \\\left(r, p p^{\prime}\right)=1}} \frac{\left|W_{3}\left(\mathbf{d}, \mathbf{0}, \mathbf{0}, p, p^{\prime}, r, 0\right)\right|}{r^{6} d_{1} d_{2} d_{3}}
$$

where

$$
\begin{aligned}
& W_{3}\left(\mathbf{d}, \mathbf{n}, \mathbf{l}, p, p^{\prime}, r, v\right)=\sum_{s\left(p p^{\prime}\right)^{*}}\left(\frac{s}{p p^{\prime}}\right) e_{p p^{\prime}}(-s N) \\
& \quad \times \sum_{\substack{a(r p)^{*} \\
a p^{\prime}+s r \equiv 0(p)}} e_{r p}(\bar{a} v) S_{\mathbf{d}}\left(r p^{\prime},\left(a p^{\prime}+s r\right) p^{-1}, \mathbf{n}\right) S_{\mathbf{d}}(r p,-a,-\mathbf{l}) .
\end{aligned}
$$

We use (69) and Lemma 2 (ii) and (iii) to get

$$
W_{3}\left(\mathbf{d}, \mathbf{n}, \mathbf{l}, p, p^{\prime}, r, v\right) \ll r^{4} R^{5} \xi(r, \mathbf{d}) .
$$

From $(72),(173),(175)$ and Lemma 15 we obtain

$$
\mathcal{V}_{p^{2}} \ll P^{2+3 \varepsilon} R^{2} \mathcal{Y}\left(P R^{-1}\right) \ll P^{3+10 \varepsilon} R D^{6} .
$$

To estimate $D_{p^{2}}$ we apply (174), (175) and Lemma 14 . We get

$$
D_{p^{2}} \ll P^{4+3 \varepsilon} Q^{-1} D^{3} R \text {. }
$$

Consider $\mathcal{V}_{p p^{\prime}}$ and $D_{p p^{\prime}}$. If $\left(a p p^{\prime}+s q, q p p^{\prime}\right)=p p^{\prime}$ then $p p^{\prime} \mid q$. We put $q=r p p^{\prime}$ and find

$$
\begin{aligned}
\mathcal{V}_{p p^{\prime}} & \ll P^{2+2 \varepsilon} R^{-9} \sum_{(\mathcal{R})} \sum_{(\mathcal{D})}\left(d_{1} d_{2} d_{3}\right)^{-1} \sum_{r \leq P R^{-2}} r^{-4} \\
& \times \sum_{\mathbf{n} \in \mathcal{N}_{\mathbf{d}, r}} \sum_{\mathbf{l} \in \mathcal{N}_{\mathbf{d}, r}} \sum_{|v| \leq P} \frac{\left|W_{4}\left(\mathbf{d}, \mathbf{n}, \mathbf{l}, p, p^{\prime}, r, v\right)\right|}{(1+|v|) \lambda(\mathbf{d}, \mathbf{n}, \mathbf{l})},
\end{aligned}
$$

and

$$
D_{p p^{\prime}} \ll P^{4} R^{-13} \sum_{(\mathcal{R})} \sum_{(\mathcal{D})} \sum_{Q(2 R)^{-2}<r \leq P R^{-2}} \frac{\left|W_{4}\left(\mathbf{d}, \mathbf{0}, \mathbf{0}, p, p^{\prime}, r, 0\right)\right|}{r^{6} d_{1} d_{2} d_{3}},
$$


where

$$
\begin{aligned}
& W_{4}\left(\mathbf{d}, \mathbf{n}, \mathbf{l}, p, p^{\prime}, r, v\right)=\sum_{s\left(p p^{\prime}\right)^{*}}\left(\frac{s}{p p^{\prime}}\right) e_{p p^{\prime}}(-s N) \\
& \quad \times \sum_{\substack{a\left(r p p^{\prime}\right)^{*} \\
\left(a+s r, r p p^{\prime}\right)=1}} e_{r p p^{\prime}}(\bar{a} v) S_{\mathbf{d}}\left(r p p^{\prime}, a+s r, \mathbf{n}\right) S_{\mathbf{d}}\left(r p p^{\prime},-a,-\mathbf{l}\right) .
\end{aligned}
$$

Using (69) and Lemma 2 (ii) and (iii) we get

$$
W_{4}\left(\mathbf{d}, \mathbf{n}, \mathbf{l}, p, p^{\prime}, r, v\right) \ll R^{10} r^{4} \xi(r, \mathbf{d}) .
$$

From (72), (178), (180) and Lemma 15 we get

$$
\mathcal{V}_{p p^{\prime}} \ll P^{2+3 \varepsilon} R^{3} \mathcal{Y}\left(P R^{-2}\right) \ll P^{3+10 \varepsilon} R D^{6} .
$$

Similarly, using (179), (180) and Lemma 14 we obtain

$$
D_{p p^{\prime}} \ll P^{4+3 \varepsilon} Q^{-1} D^{3} R .
$$

Consider $\mathcal{V}_{p^{2} p^{\prime}}$ and $D_{p^{2} p^{\prime}}$. If $\left(a p p^{\prime}+s q, q p p^{\prime}\right)=\delta=p^{2} p^{\prime}$ then the conditions $\left|n_{i}\right| \leq 4 d_{i} R^{2} \delta^{-1} P^{\varepsilon}$ imply $\mathbf{n}=\mathbf{0}$. We have also $p p^{\prime} \mid q, p^{2} \nmid q$. We put $q=r p p^{\prime}$ and find

$$
\begin{aligned}
\mathcal{V}_{p^{2} p^{\prime}} & \ll P^{2+2 \varepsilon} R^{-6} \sum_{(\mathcal{R})} \sum_{(\mathcal{D})}\left(d_{1} d_{2} d_{3}\right)^{-1} \sum_{\substack{r \leq P R^{-2} \\
(r, p)=1}} r^{-4} \\
& \times \sum_{\mathbf{l} \in \mathcal{N}_{\mathbf{d}, r}} \sum_{|v| \leq P} \frac{\left|W_{5}\left(\mathbf{d}, \mathbf{l}, p, p^{\prime}, r, v\right)\right|}{(1+|v|) \lambda(\mathbf{d}, \mathbf{0}, \mathbf{l})}
\end{aligned}
$$

and

$$
D_{p^{2} p^{\prime}} \ll P^{4} R^{-10} \sum_{(\mathcal{R})} \sum_{(\mathcal{D})} \sum_{\substack{Q\left(p p^{\prime}\right)^{-1}<r \leq P R^{-2} \\(r, p)=1}} \frac{\left|W_{5}\left(\mathbf{d}, \mathbf{0}, p, p^{\prime}, r, 0\right)\right|}{r^{6} d_{1} d_{2} d_{3}},
$$

where

$$
\begin{aligned}
& W_{5}\left(\mathbf{d}, \mathbf{l}, p, p^{\prime}, r, v\right)=\sum_{s\left(p p^{\prime}\right)^{*}}\left(\frac{s}{p p^{\prime}}\right) e_{p p^{\prime}}(-s N) \\
& \quad \times \sum_{\substack{a\left(r p p^{\prime}\right)^{*} \\
\left(a+s r, r p p^{\prime}\right)=p}} e_{r p p^{\prime}}(\bar{a} v) S_{\mathbf{d}}\left(r p^{\prime},(a+s r) p^{-1}\right) S_{\mathbf{d}}\left(r p p^{\prime},-a,-\mathbf{l}\right) .
\end{aligned}
$$

We note again that the set (160) is a reduced system of residues modulo $p p^{\prime}$. Similarly, the set

$$
\left\{b p+h r p^{\prime} \mid b\left(r p^{\prime}\right)^{*}, h(p)^{*}\right\}
$$


is a reduced system of residues modulo $r p p^{\prime}$.

If $s=c p^{\prime}+c^{\prime} p$ and $a=b p+h r p^{\prime}$ then the condition $\left(a+s r, r p p^{\prime}\right)=p$ is equivalent to the system of conditions $\left(b+c^{\prime} r, r p^{\prime}\right)=1$ and $h+c \equiv 0(p)$ and we have

$$
S_{\mathbf{d}}\left(r p^{\prime},(a+s r) p^{-1}\right)=S_{\mathbf{d}}\left(r p^{\prime}, b+c^{\prime} r\right) .
$$

Furthermore, using Lemma 5 we also get

$$
S_{\mathbf{d}}\left(r p^{\prime} p,-a,-\mathbf{l}\right)=S_{\mathbf{d}}\left(r p^{\prime},-b p^{2},-\mathbf{l}\right) S_{\mathbf{d}}\left(p,-h\left(r p^{\prime}\right)^{2},-\mathbf{l}\right) .
$$

From (127) and Lemma $2(i i)-(i v)$ we obtain

$$
\begin{aligned}
W_{5}\left(\mathbf{d}, \mathbf{l}, p, p^{\prime}, r, v\right)= & \left(\frac{p}{p^{\prime}}\right)\left(\frac{p^{\prime}}{p}\right) S^{3}(p, 1) \\
& \times K\left(p,-N,-\overline{\left(r p^{\prime}\right)^{2}}\left(v+\sum_{i=1}^{3} \overline{\left(4 d_{i}^{2}\right)} l_{i}^{2}\right)\right) \\
& \times \sum_{c^{\prime}\left(p^{\prime}\right)}\left(\frac{c^{\prime}}{p^{\prime}}\right) e_{p^{\prime}}\left(-c^{\prime} N\right) \sum_{\begin{array}{c}
b\left(r p^{\prime}\right)^{*} \\
\left(b+c^{\prime} r, r p^{\prime}\right)=1
\end{array}} e_{r p^{\prime}}\left(\overline{\left(b p^{2}\right)} v\right) \\
& \times S_{\mathbf{d}}\left(r p^{\prime}, b+c^{\prime} r\right) S_{\mathbf{d}}\left(r p^{\prime},-b p^{2},-\mathbf{l}\right) \\
\ll & R^{7} r^{4} \xi(r, \mathbf{d}) .
\end{aligned}
$$

We apply (183), (185) and Lemmas 13 and 14 to get

$$
\mathcal{V}_{p^{2} p^{\prime}} \ll P^{3+10 \varepsilon} R D^{6} .
$$

Analogously, from (184), (185) and Lemma 14 we obtain

$$
D_{p^{2} p^{\prime}} \ll P^{4+5 \varepsilon} Q^{-1} D^{3} R .
$$

Consider $\mathcal{V}_{\left(p p^{\prime}\right)^{2}}$ and $D_{\left(p p^{\prime}\right)^{2}}$. If $\left(a p p^{\prime}+s q, q p p^{\prime}\right)=\delta=\left(p p^{\prime}\right)^{2}$ then the conditions $\left|n_{i}\right| \leq 4 d_{i} R^{2} \delta^{-1} P^{\varepsilon}$ imply $\mathbf{n}=\mathbf{0}$. We have also $p p^{\prime} \mid q, p^{2} \nmid q, p^{\prime 2} \nmid q$. We put $q=r p p^{\prime}$ and find

$$
\begin{aligned}
\mathcal{V}_{\left(p p^{\prime}\right)^{2}} & \ll P^{2+2 \varepsilon} R^{-3} \sum_{(\mathcal{R})} \sum_{(\mathcal{D})}\left(d_{1} d_{2} d_{3}\right)^{-1} \sum_{\substack{r \leq P R^{-2} \\
\left(r, p p^{\prime}\right)=1}} r^{-4} \\
& \times \sum_{\mathbf{l} \in \mathcal{N}_{\mathbf{d}, r}} \sum_{|v| \leq P} \frac{\left|W_{6}\left(\mathbf{d}, \mathbf{l}, p, p^{\prime}, r, v\right)\right|}{(1+|v|) \lambda(\mathbf{d}, \mathbf{0}, \mathbf{l})}
\end{aligned}
$$

and

$$
D_{\left(p p^{\prime}\right)^{2}} \ll P^{4} R^{-7} \sum_{(\mathcal{R})} \sum_{(\mathcal{D})} \sum_{\substack{Q\left(p p^{\prime}\right)^{-1}<r \leq P R^{-2} \\\left(r, p p^{\prime}\right)=1}} \frac{\left|W_{6}\left(\mathbf{d}, \mathbf{0}, p, p^{\prime}, r, 0\right)\right|}{r^{6} d_{1} d_{2} d_{3}},
$$


where

$$
\begin{aligned}
& W_{6}\left(\mathbf{d}, \mathbf{l}, p, p^{\prime}, r, v\right)=\sum_{s\left(p p^{\prime}\right)^{*}}\left(\frac{s}{p p^{\prime}}\right) e_{p p^{\prime}}(-s N) \\
& \times \sum_{\substack{a\left(r p p^{\prime}\right)^{*} \\
a+s r \equiv 0\left(p p^{\prime}\right)}} e_{r p p^{\prime}}(\bar{a} v) S_{\mathbf{d}}\left(r,(a+s r)\left(p p^{\prime}\right)^{-1}\right) S_{\mathbf{d}}\left(r p p^{\prime},-a,-\mathbf{l}\right) .
\end{aligned}
$$

We note that the set $\left\{b p p^{\prime}+t r \mid b(r)^{*}, t\left(p p^{\prime}\right)^{*}\right\}$ is a reduced system of residues modulo $r p p^{\prime}$. If $a=b p p^{\prime}+t r$ then the condition $a+s r \equiv 0\left(p p^{\prime}\right)$ is equivalent to $t+s \equiv 0\left(p p^{\prime}\right)$. We also have $(a+s r)\left(p p^{\prime}\right)^{-1} \equiv b(r)$ and

$$
S_{\mathbf{d}}\left(r p p^{\prime},-a,-\mathbf{l}\right)=S_{\mathbf{d}}\left(r,-b\left(p p^{\prime}\right)^{2},-\mathbf{l}\right) S_{\mathbf{d}}\left(p p^{\prime},-t r^{2},-\mathbf{l}\right) .
$$

We apply (127) and Lemma $2(i i)-(i v)$ to find that

$$
\begin{aligned}
W_{6}\left(\mathbf{d}, \mathbf{l}, p, p^{\prime}, r, v\right)= & S^{3}\left(p p^{\prime}, 1\right) K\left(p p^{\prime},-N,-\overline{r^{2}}\left(v+\sum_{i=1}^{3} \overline{\left(4 d_{i}^{2}\right)} l_{i}^{2}\right)\right) \\
& \times \sum_{b(r)^{*}} e_{r}\left(\overline{b\left(p p^{\prime}\right)^{2}} v\right) S_{\mathbf{d}}(r, b) S_{\mathbf{d}}\left(r,-b\left(p p^{\prime}\right)^{2},-\mathbf{l}\right) \\
& \ll R^{4} r^{4} \xi(r, \mathbf{d}) .
\end{aligned}
$$

We use (188), (190) and Lemmas 13 and 14 to find

$$
\mathcal{V}_{\left(p p^{\prime}\right)^{2}} \ll P^{3+10 \varepsilon} R D^{6}
$$

From (189), (190) and Lemma 14 we obtain

$$
D_{\left(p p^{\prime}\right)}{ }^{2} \ll P^{4+5 \varepsilon} Q^{-1} D^{3} R
$$

Conclusion. Using (122), (153), (170), (176), (181), (186) and (191) we find that

$$
\mathcal{V}^{*} \ll\left(P^{3} R+P^{2} R^{5}\right) D^{6} P^{15 \varepsilon}
$$

Respectively, using (123), (154), (172), (177), (182), (187) and (192) we obtain

$$
D^{*} \ll P^{4+5 \varepsilon} Q^{-1} D^{3} R .
$$

\subsubsection{The estimation of $E^{*}$.}

Consider the sum $\mathcal{F}$, defined by (117). We divide it into parts according to the congruence class of $p p^{\prime}\left(a_{1} q_{2}+a_{2} q_{1}\right)+s q_{1} q_{2}$ modulo $q_{1} q_{2} p p^{\prime}$. After some 
rearrangements we get

$$
\begin{aligned}
\mathcal{F}= & \sum_{0<|l| \leq 4 R^{2} Q^{2} P^{\varepsilon-2}} \sum_{\substack{q_{1}, q_{2} \leq Q \\
|l|\left(p p^{\prime}\right)^{-1} P^{2-\varepsilon}<q_{1} q_{2}}}\left(q_{1} q_{2}\right)^{-3} \\
& \times e\left(\frac{n l}{q_{1} q_{2} p p^{\prime}}\right) \sum_{\substack{s\left(p p^{\prime}\right)^{*}\\
}}\left(\frac{s}{p p^{\prime}}\right) e_{p p^{\prime}}(-s N) \\
& \times \sum_{\substack{a_{1}\left(q_{1}\right)^{*}, a_{2}\left(q_{2}\right)^{*} \\
p p^{\prime}\left(a_{1} q_{2}+a_{2} q_{1}\right)+s q_{1} q_{2} \equiv l\left(q_{1} q_{2} p p^{\prime}\right)}} S_{\mathbf{d}\left(q_{1},-a_{1}\right) S_{\mathbf{d}}\left(q_{2},-a_{2}\right) .}
\end{aligned}
$$

From this point onwards we assume that

$$
Q \leq P^{1-\varepsilon} R^{-1 / 2} .
$$

This inequality implies that in the right hand side of (195) we sum over $l$ satisfying $0<|l|<R$, consequently $\left(l, p p^{\prime}\right)=1$ and $\left(q_{1} q_{2}, p p^{\prime}\right)=1$. Therefore, the congruence condition in the sum over $a_{1}, a_{2}$ is equivalent to the system of congruences

$$
p p^{\prime}\left(a_{1} q_{2}+a_{2} q_{1}\right) \equiv l\left(q_{1} q_{2}\right), \quad s q_{1} q_{2} \equiv l\left(p p^{\prime}\right)
$$

The second congruence determines $s$ uniquely modulo $p p^{\prime}$. Therefore, the sum over $s$ in the right hand side of (195) has exactly one term corresponding to $s \equiv l \overline{q_{1} q_{2}}\left(p p^{\prime}\right)$. Hence we obtain

$$
\begin{aligned}
\mathcal{F}= & \sum_{\substack{0<|l| \leq 4 R^{2} Q^{2} P^{\varepsilon-2} \\
\left(l, p p^{\prime}\right)=1}} \sum_{\substack{q_{1}, q_{2} \leq Q \\
|l|\left(p p^{\prime}\right)^{-1} P^{2}-\varepsilon<q_{1} q_{2}}}^{\left(q_{1} q_{2}, p p^{\prime}\right)=1} \\
& \times\left(\frac{q_{1} q_{2} l}{p p^{\prime}}\right) \cdot e\left(\frac{-N l \overline{q_{1} q_{2}}}{p p^{\prime}}\right) \cdot \mathcal{S},
\end{aligned}
$$

where

$$
\mathcal{S}=\sum_{\substack{a_{1}\left(q_{1}\right)^{*}, a_{2}\left(q_{2}\right)^{*} \\ p p^{\prime}\left(a_{1} q_{2}+a_{2} q_{1}\right) \equiv l\left(q_{1} q_{2}\right)}} S_{\mathbf{d}}\left(q_{1},-a_{1}\right) S_{\mathbf{d}}\left(q_{2},-a_{2}\right) .
$$

We represent the integers $q_{i}$ as

$$
q_{i}=g_{i} b_{i}, \quad i=1,2,
$$

where

$$
g_{i}=\prod_{\substack{p^{l} \| q_{i} \\ p \nmid 2 d_{1} d_{2} d_{3}\left(q_{1}, q_{2}\right)}} p^{l}, \quad i=1,2
$$


and where $b_{i}$ are determined by (199). To produce the set of summation over $q_{1}, q_{2}$ in the right hand side of (197) we have to sum over the set of all quadruples of integers $g_{1}, g_{2}, b_{1}, b_{2}$, satisfying the conditions

$$
\begin{aligned}
& g_{1} b_{1}, g_{2} b_{2} \leq Q, \quad|l|\left(p p^{\prime}\right)^{-1} P^{2-\varepsilon}<b_{1} b_{2} g_{1} g_{2}, \\
& \left(g_{1} g_{2}, 2 p p^{\prime} d_{1} d_{2} d_{3} b_{1} b_{2}\right)=\left(g_{1}, g_{2}\right)=\left(b_{1} b_{2}, p p^{\prime}\right)=1, \\
& \rho_{\mathbf{d}}\left(b_{1}\right)=\rho_{\mathbf{d}}\left(b_{2}\right),
\end{aligned}
$$

where we have defined

$$
\rho_{\mathbf{d}}(m)=\prod_{\substack{p \mid m \\ p \nmid 2 d_{1} d_{2} d_{3}}} p .
$$

Consider the sum $\mathcal{S}$. Suppose that $q_{1}, q_{2}$ satisfy (199) and (201). We note that the sets $\left\{\alpha_{i} b_{i}+\beta_{i} g_{i} \mid \alpha_{i}\left(g_{i}\right)^{*}, \beta_{i}\left(b_{i}\right)^{*}\right\}, i=1,2$ are reduced systems of residues modulo $q_{i}, i=1,2$. Furthermore, if $a_{i}=\alpha_{i} b_{i}+\beta_{i} g_{i}, i=1,2$, then the congruence condition, imposed on the domain of summation in (198), is equivalent to the system of the following three conditions

$$
\begin{gathered}
p p^{\prime} \alpha_{1} b_{1} b_{2} g_{2} \equiv l\left(g_{1}\right), \quad p p^{\prime} \alpha_{2} b_{1} b_{2} g_{1} \equiv l\left(g_{2}\right), \\
p p^{\prime} g_{1} g_{2}\left(\beta_{1} b_{2}+\beta_{2} b_{1}\right) \equiv l\left(b_{1} b_{2}\right) .
\end{gathered}
$$

We use Lemma $2(i)$ and $(i v)$ to find that under conditions (199), (201) we have

$$
\begin{aligned}
\mathcal{S}= & \sum_{\substack{\alpha_{1}\left(g_{1}\right)^{*}, \alpha_{2}\left(g_{2}\right)^{*} \\
(203)}} S^{3}\left(g_{1},-\alpha_{1}\right) S^{3}\left(g_{2},-\alpha_{2}\right) \cdot \mathcal{S}^{\prime} \\
& =\left(\frac{-p p^{\prime} b_{1} b_{2} g_{2} l}{g_{1}}\right)\left(\frac{-p p^{\prime} b_{1} b_{2} g_{1} l}{g_{2}}\right) S^{3}\left(g_{1}, 1\right) S^{3}\left(g_{2}, 1\right) \cdot \mathcal{S}^{\prime}
\end{aligned}
$$

where

$$
\mathcal{S}^{\prime}=\sum_{\substack{\beta_{1}\left(b_{1}\right)^{*}, \beta_{2}\left(b_{2}\right)^{*} \\(204)}} S_{\mathbf{d}}\left(b_{1},-\beta_{1}\right) S_{\mathbf{d}}\left(b_{2},-\beta_{2}\right) .
$$

We decompose $\mathcal{F}$ into $\mathcal{O}\left(\log ^{2} P\right)$ sums $\mathcal{F}\left(G_{1}, G_{2}\right)$ according to the size of $g_{i}$. In $\mathcal{F}\left(G_{1}, G_{2}\right)$ we sum over

$$
g_{i} \in\left(G_{i}, 2 G_{i}\right], i=1,2 .
$$

Consider the sum $E^{*}$, defined by $(119)$, and denote by $E\left(G_{1}, G_{2}\right)$ the contribution to $E^{*}$ coming from $\mathcal{F}\left(G_{1}, G_{2}\right)$. We have

$$
E^{*} \ll P^{\varepsilon} \max _{G_{1}, G_{2} \leq Q}\left|E\left(G_{1}, G_{2}\right)\right|
$$


and

$$
E\left(G_{1}, G_{2}\right) \ll P^{2} R^{-1} \sum_{(\mathcal{R})} \sum_{(\mathcal{D})}\left(d_{1} d_{2} d_{3}\right)^{-1} \sum_{0<n<N}\left|\mathcal{F}\left(G_{1}, G_{2}\right)\right| .
$$

Consider $\mathcal{F}\left(G_{1}, G_{2}\right)$. We take into account (197), (199) - (201), (205), (207) and find that

$$
\begin{aligned}
\mathcal{F}\left(G_{1}, G_{2}\right)= & \sum_{\substack{0<|l| \leq 4 R^{2} Q^{2} P^{\varepsilon-2} \\
\left(l, p p^{\prime}\right)=1}} \sum_{\substack{g_{1}, g_{2}, b_{1}, b_{2} \\
(201),(207)}}\left(b_{1} b_{2} g_{1} g_{2}\right)^{-3} \\
& \times e\left(\frac{n l}{g_{1} g_{2} b_{1} b_{2} p p^{\prime}}\right) \cdot\left(\frac{g_{1} g_{2} b_{1} b_{2} l}{p p^{\prime}}\right) \cdot e\left(\frac{-N l \overline{\left(b_{1} b_{2} g_{1} g_{2}\right)}}{p p^{\prime}}\right) \\
& \times\left(\frac{-p p^{\prime} b_{1} b_{2} g_{2} l}{g_{1}}\right) \cdot\left(\frac{-p p^{\prime} b_{1} b_{2} g_{1} l}{g_{2}}\right) \\
& \times S^{3}\left(g_{1}, 1\right) S^{3}\left(g_{2}, 1\right) \cdot \mathcal{S}^{\prime},
\end{aligned}
$$

where $\mathcal{S}^{\prime}$ is defined by (206) and where the summation over $g_{1}, g_{2}, b_{1}, b_{2}$ is restricted to integers satisfying (201) and (207).

We split further $b_{1}=B_{1} \Delta, b_{2}=B_{2} \Delta$, where $\Delta=\left(b_{1}, b_{2}\right)$. The congruence condition (204) implies $\Delta \mid l$, so we put $l=\Delta v$. Now we can simplify this congruence and we write $\mathcal{S}^{\prime}$ in the form

$$
\mathcal{S}^{\prime}=\Xi\left(p p^{\prime} g_{1} g_{2}\right),
$$

where

$$
\Xi(\mu)=\Xi\left(B_{1}, B_{2}, \Delta, v, \mu\right)
$$

We obtain

$$
\begin{aligned}
& \mathcal{F}\left(G_{1}, G_{2}\right)=\sum_{\substack{0<\Delta|v| \leq 4 R^{2} Q^{2} P^{\varepsilon-2} \\
\left(\Delta v, p p^{\prime}\right)=1}} \sum_{\substack{g_{1}, g_{2}, B_{1}, B_{2} \\
(213)}} \frac{S^{3}\left(g_{1}, 1\right) S^{3}\left(g_{2}, 1\right)}{\left(B_{1} B_{2} \Delta^{2} g_{1} g_{2}\right)^{3}} \\
& \quad \times e\left(\frac{n v}{B_{1} B_{2} \Delta g_{1} g_{2} p p^{\prime}}\right) \cdot\left(\frac{B_{1} B_{2} g_{1} g_{2} \Delta v}{p p^{\prime}}\right) \cdot e\left(\frac{-N v \overline{\left(B_{1} B_{2} \Delta g_{1} g_{2}\right)}}{p p^{\prime}}\right) \\
& \times\left(\frac{-p p^{\prime} B_{1} B_{2} g_{2} \Delta v}{g_{1}}\right) \cdot\left(\frac{-p p^{\prime} B_{1} B_{2} g_{1} \Delta v}{g_{2}}\right) \cdot \Xi\left(p p^{\prime} g_{1} g_{2}\right) .
\end{aligned}
$$

In the formula above the summation over $g_{1}, g_{2}, B_{1}, B_{2}$ is restricted to integers satisfying the conditions

$$
\begin{aligned}
& B_{1} \Delta g_{1} \leq Q, \quad B_{2} \Delta g_{2} \leq Q, \quad G_{1}<g_{1} \leq 2 G_{1}, \quad G_{2}<g_{2} \leq 2 G_{2} \\
& |v|\left(p p^{\prime}\right)^{-1} P^{2-\varepsilon}<B_{1} B_{2} \Delta g_{1} g_{2}, \quad \rho_{\mathbf{d}}\left(\Delta B_{1}\right)=\rho_{\mathbf{d}}\left(\Delta B_{2}\right)=\rho_{\mathbf{d}}(\Delta) \\
& \left(g_{1} g_{2}, 2 p p^{\prime} d_{1} d_{2} d_{3} \Delta B_{1} B_{2}\right)=\left(g_{1}, g_{2}\right)=\left(B_{1} B_{2} \Delta, p p^{\prime}\right)=\left(B_{1}, B_{2}\right)=1 .
\end{aligned}
$$


Here we have to verify that if $\rho_{\mathbf{d}}\left(\Delta B_{1}\right)=\rho_{\mathbf{d}}\left(\Delta B_{2}\right)$ then the common value of these integers equals $\rho_{\mathbf{d}}(\Delta)$. This follows easily from the definition of $\rho_{\mathbf{d}}$ and from the properties of the integers involved.

We shall estimate $E\left(G_{1}, G_{2}\right)$ by two different ways.

The first estimate for $E\left(G_{1}, G_{2}\right)$. Using Lemma 2 (ii) and (iii) we find that

$$
S_{\mathbf{d}}\left(\Delta B_{j},-\beta_{j}\right) \ll\left(\Delta B_{j}\right)^{3 / 2} d_{1} d_{2} d_{3}
$$

and it is easy to see that if $\left(\mu, \Delta B_{1} B_{2}\right)=1$ then

$$
\sum_{\substack{\beta_{1}\left(\Delta B_{1}\right)^{*}, \beta_{2}\left(\Delta B_{2}\right)^{*} \\ \mu\left(\beta_{1} B_{2}+\beta_{2} B_{1}\right) \equiv v\left(\Delta B_{1} B_{2}\right)}} 1 \ll \Delta .
$$

From (211), (214) and (215) we get

$$
\Xi(\mu) \ll \Delta^{4}\left(B_{1} B_{2}\right)^{3 / 2}\left(d_{1} d_{2} d_{3}\right)^{2} \quad \text { if } \quad\left(\mu, \Delta B_{1} B_{2}\right)=1 .
$$

Applying Lemma 2 (iii) we get

$$
\sum_{G_{i}<g_{i} \leq 2 G_{i}} g_{i}^{-3}\left|S^{3}\left(g_{i}, 1\right)\right| \ll \sum_{G_{i}<g_{i} \leq 2 G_{i}} g_{i}^{-3 / 2} \ll G_{i}^{-1 / 2}, \quad i=1,2 .
$$

From (209), (212), (216) and (217) we obtain

$$
E\left(G_{1}, G_{2}\right) \ll E^{\#}\left(G_{1}, G_{2}\right),
$$

where

$$
E^{\#}\left(G_{1}, G_{2}\right)=P^{4} R\left(G_{1} G_{2}\right)^{-1 / 2} \sum_{(\mathcal{D})} d_{1} d_{2} d_{3} \mathcal{T}
$$

and

$$
\mathcal{T}=\sum_{\substack{\Delta v \leq 4 R^{2} Q^{2} P^{\varepsilon-2} \\ \Delta, v>0}} \Delta^{-2} \sum_{B_{1}, B_{2}(221)}\left(B_{1} B_{2}\right)^{-3 / 2} .
$$

Here we sum over integers $B_{1}$ and $B_{2}$ satisfying

$$
\begin{aligned}
& B_{1} \leq Q \Delta^{-1} G_{1}^{-1}, \quad B_{2} \leq Q \Delta^{-1} G_{2}^{-1}, \\
& |v|\left(16 R^{2} \Delta G_{1} G_{2}\right)^{-1} P^{2-\varepsilon} \leq B_{1} B_{2}, \quad\left(B_{1} B_{2}, p p^{\prime}\right)=1, \\
& \rho_{\mathbf{d}}\left(\Delta B_{1}\right)=\rho_{\mathbf{d}}\left(\Delta B_{2}\right)=\rho_{\mathbf{d}}(\Delta) .
\end{aligned}
$$

Using these conditions we get

$$
\begin{aligned}
\mathcal{T} & \ll \sum_{\substack{\Delta v \leq 4 R^{2} Q^{2} P^{\varepsilon-2} \\
\Delta, v>0}} \Delta^{-2}\left(v R^{-2}\left(\Delta G_{1} G_{2}\right)^{-1} P^{2-\varepsilon}\right)^{-3 / 2} \mathcal{T}_{1}^{2} \\
& \ll R^{3}\left(G_{1} G_{2}\right)^{3 / 2} P^{2 \varepsilon-3} \sum_{0<\Delta \leq 4 R^{2} Q^{2} P^{\varepsilon-2}} \Delta^{-1 / 2} \mathcal{T}_{1}^{2},
\end{aligned}
$$


where

$$
\mathcal{T}_{1}=\sum_{\substack{B \leq P \\ \rho_{\mathbf{d}}(\Delta B)=\rho_{\mathbf{d}}(\Delta)}} 1
$$

Consider $\mathcal{T}_{1}$. If $\rho_{\mathbf{d}}(\Delta B)=\rho_{\mathbf{d}}(\Delta)$ then every prime factor of $B$ divides also $2 d_{1} d_{2} d_{3} \Delta$. Hence

$$
\begin{aligned}
\mathcal{T}_{1} & \leq \sum_{\substack{B=1 \\
p|B \Rightarrow p| 2 d_{1} d_{2} d_{3} \Delta}}^{\infty}\left(P B^{-1}\right)^{\varepsilon}=P^{\varepsilon} \prod_{p \mid 2 d_{1} d_{2} d_{3} \Delta}\left(1+p^{-\varepsilon}+p^{-2 \varepsilon}+\ldots\right) \\
& \leq P^{\varepsilon}\left(1+2^{-\varepsilon}+2^{-2 \varepsilon}+\ldots\right)^{\nu\left(2 d_{1} d_{2} d_{3} \Delta\right)} \ll P^{2 \varepsilon} .
\end{aligned}
$$

We substitute this estimate for $\mathcal{T}_{1}$ in $(222)$ and then use (219) to get

$$
E^{\#}\left(G_{1}, G_{2}\right) \ll G_{1} G_{2} R^{5} Q D^{6} P^{10 \varepsilon} .
$$

From this inequality and (218) we obtain

$$
E\left(G_{1}, G_{2}\right) \ll G_{1} G_{2} R^{5} Q D^{6} P^{10 \varepsilon} .
$$

The second estimate for $E\left(G_{1}, G_{2}\right)$. Suppose that

$$
G_{1} \leq G_{2}
$$

We use (209), (211), (212), and Lemma 2 (iii) to get

$$
\begin{aligned}
E\left(G_{1}, G_{2}\right) \ll P^{2} R^{-1} \sum_{(\mathcal{R})} \sum_{(\mathcal{D})}\left(d_{1} d_{2} d_{3}\right)^{-1} \sum_{\substack { 0<n<N \\
\begin{subarray}{c}{0<\Delta|v| \leq 4 R^{2} Q^{2} P^{\varepsilon-2} \\
\left(\Delta v, p p^{\prime}\right)=1{ 0 < n < N \\
\begin{subarray} { c } { 0 < \Delta | v | \leq 4 R ^ { 2 } Q ^ { 2 } P ^ { \varepsilon - 2 } \\
( \Delta v , p p ^ { \prime } ) = 1 } }\end{subarray}} \sum^{\times \sum_{B_{1}, B_{2}(221)}\left(B_{1} B_{2} \Delta^{2}\right)^{-3}} \\
\times \sum_{\substack{G_{1}<g_{1} \leq 2 G_{1} \\
\left(g_{1}, 2 p p^{\prime} d_{1} d_{2} d_{3} \Delta B_{1} B_{2}\right)=1}} g_{1}^{-3 / 2} \max _{\substack{G, G^{\prime}: \\
G_{2} \leq G \leq G^{\prime} \leq 2 G_{2}}}\left|\mathcal{M}_{0}\right|,
\end{aligned}
$$

where

$$
\begin{aligned}
\mathcal{M}_{0}= & \sum_{\substack{G<g \leq G^{\prime} \\
\left(g, 2 p p^{\prime} d_{1} d_{2} d_{3} \Delta B_{1} B_{2} g_{1}\right)=1}} g^{-3} S^{3}(g, 1) \cdot e\left(\frac{n v}{B_{1} B_{2} \Delta g_{1} g p p^{\prime}}\right) \\
& \times\left(\frac{g}{p p^{\prime}}\right) \cdot e\left(\frac{-N v \overline{\left(B_{1} B_{2} \Delta g_{1} g\right)}}{p p^{\prime}}\right) \cdot\left(\frac{g}{g_{1}}\right) \cdot\left(\frac{-p p^{\prime} B_{1} B_{2} g_{1} \Delta v}{g}\right) \cdot \Xi\left(p p^{\prime} g_{1} g\right) .
\end{aligned}
$$

We have

$$
\mathcal{M}_{0}=\sum_{\lambda\left(\Delta B_{1} B_{2}\right)^{*}} \Xi\left(p p^{\prime} g_{1} \lambda\right) \mathcal{M}_{1}
$$


where

$$
\begin{aligned}
\mathcal{M}_{1}= & \sum_{\substack{\left.G<g \leq G^{\prime} \\
g, 2 p p^{\prime} d_{1} d_{2} d_{3} g_{1}\right)=1 \\
g \equiv \lambda\left(\Delta B_{1} B_{2}\right)}} g^{-3} S^{3}(g, 1) e\left(\frac{n v}{B_{1} B_{2} \Delta g_{1} g p p^{\prime}}\right) \cdot\left(\frac{g}{p p^{\prime}}\right) \\
& \times e\left(\frac{-N v \overline{\left(B_{1} B_{2} \Delta g_{1} g\right)}}{p p^{\prime}}\right) \cdot\left(\frac{g}{g_{1}}\right) \cdot\left(\frac{-p p^{\prime} B_{1} B_{2} g_{1} \Delta v}{g}\right) .
\end{aligned}
$$

From (216) and (229) we get

$$
\mathcal{M}_{0} \ll \Delta^{4}\left(B_{1} B_{2}\right)^{3 / 2}\left(d_{1} d_{2} d_{3}\right)^{2} \sum_{\lambda\left(\Delta B_{1} B_{2}\right)^{*}}\left|\mathcal{M}_{1}\right| .
$$

We divide $\mathcal{M}_{1}$ into four parts according to the congruence class of $g$ modulo 8 . We have

$$
\mathcal{M}_{1} \ll \max _{j=1,3,5,7}\left|\mathcal{M}_{2}(j)\right|,
$$

where the summation in $\mathcal{M}_{2}(j)$ is restricted to $g \equiv j(8)$.

Define the natural numbers $B_{1}^{\prime}, B_{2}^{\prime}, \Delta^{\prime}, v^{\prime}$ by $\left(B_{1}^{\prime} B_{2}^{\prime} \Delta^{\prime} v^{\prime}, 2\right)=1$ and $B_{1}=$ $2^{\mu_{1}} B_{1}^{\prime}, B_{2}=2^{\mu_{2}} B_{2}^{\prime}, \Delta=2^{\mu_{3}} \Delta^{\prime},|v|=2^{\mu_{4}} v^{\prime}$. Consider any of the sums $\mathcal{M}_{2}(j)$. We use Lemmas $2(i v)$ and 7 to get

$$
\left|\mathcal{M}_{2}(j)\right|=\left|\mathcal{M}_{3}\right|,
$$

where

$$
\begin{aligned}
\mathcal{M}_{3}= & \sum_{\substack{\left.G<g \leq G^{\prime} \\
g, p p^{\prime} d_{1} d_{2} d_{3} g_{1}\right)=1 \\
g \equiv \lambda\left(\Delta B_{1} B_{2}\right) \\
g \equiv j(8)}} g^{-3 / 2} e\left(\frac{n v}{B_{1} B_{2} \Delta g_{1} g p p^{\prime}}\right) \\
& \times e\left(\frac{-N v \overline{\left(B_{1} B_{2} \Delta g_{1} g\right)}}{p p^{\prime}}\right)\left(\frac{B_{1}^{\prime} B_{2}^{\prime} \Delta^{\prime} v^{\prime}}{g}\right) .
\end{aligned}
$$

We may assume that $j \equiv \lambda\left(\left(8, B_{1} B_{2} \Delta\right)\right)$ because otherwise the sum $\mathcal{M}_{3}$ would be empty. Denote

$$
T=\left[8, B_{1} B_{2} \Delta\right] .
$$

There exists $\pi$ satisfying $(\pi, T)=1$ and such that the system of congruences $g \equiv \lambda\left(\Delta B_{1} B_{2}\right), g \equiv j(8)$ is equivalent to $g \equiv \pi(T)$. We apply again Lemma 7 to get

$$
\left|\mathcal{M}_{3}\right|=\left|\mathcal{M}_{4}\right|,
$$

where

$$
\mathcal{M}_{4}=\sum_{\substack{\left.G<g \leq G^{\prime} \\ g, p \prime^{\prime} d_{1} d_{2} d_{3} g_{1}\right)=1 \\ g \equiv \pi(T)}} g^{-3 / 2} e\left(\frac{n v}{B_{1} B_{2} \Delta g_{1} g p p^{\prime}}\right) e\left(\frac{-N v \overline{\left(B_{1} B_{2} \Delta g_{1} g\right)}}{p p^{\prime}}\right)\left(\frac{g}{v^{\prime}}\right) .
$$


Furthermore, we use (221) and easily find that

$$
\frac{d}{d x}\left(x^{-3 / 2} e\left(\frac{n v}{B_{1} B_{2} \Delta g_{1} x p p^{\prime}}\right)\right) \ll P^{\varepsilon} G_{2}^{-5 / 2}
$$

uniformly for $x \in\left[G_{2}, 2 G_{2}\right]$. We apply Abel's formula to get

$$
\mathcal{M}_{4} \ll P^{\varepsilon} G_{2}^{-3 / 2} \max _{G^{\prime \prime} \in\left[G, G^{\prime}\right]}\left|\mathcal{M}_{5}\right|,
$$

where

$$
\mathcal{M}_{5}=\sum_{\substack{\left.G<g \leq G^{\prime \prime} \\ g, p p^{\prime} d_{1} d_{2} d_{3} g_{1}\right)=1 \\ g \equiv \pi(T)}} e\left(\frac{-N v \overline{\left(B_{1} B_{2} \Delta g_{1} g\right)}}{p p^{\prime}}\right)\left(\frac{g}{v^{\prime}}\right) .
$$

Finally, to get rid of the condition $\left(g, d_{1} d_{2} d_{3} g_{1}\right)=1$, imposed on $g$, we use the well known identity for the Möbius function and obtain

$$
\begin{aligned}
\mathcal{M}_{5} & =\sum_{\substack{G<g \leq G^{\prime \prime} \\
\left(g, p p^{\prime}\right)=1 \\
g \equiv \pi(T)}}\left(\sum_{\delta \mid\left(g, d_{1} d_{2} d_{3} g_{1}\right)} \mu(\delta)\right) e\left(\frac{-N v \overline{\left(B_{1} B_{2} \Delta g_{1} g\right)}}{p p^{\prime}}\right)\left(\frac{g}{v^{\prime}}\right) \\
& =\sum_{\delta \mid d_{1} d_{2} d_{3} g_{1}} \mu(\delta) \mathcal{M}_{6}
\end{aligned}
$$

where

$$
\mathcal{M}_{6}=\sum_{\substack{\left.G<g \leq G^{\prime \prime} \\ g, p p^{\prime}\right)=1 \\ g \equiv \pi(T) \\ g \equiv 0(\delta)}} e\left(\frac{-N v \overline{\left(B_{1} B_{2} \Delta g_{1} g\right)}}{p p^{\prime}}\right)\left(\frac{g}{v^{\prime}}\right) .
$$

We have $(\pi, T)=1$. Hence we can assume that $(\delta, T)=1$ because otherwise the sum $\mathcal{M}_{6}$ would be empty. Therefore we obtain

$$
\left|\mathcal{M}_{6}\right| \leq\left|\mathcal{M}_{7}\right|
$$

where

$$
\mathcal{M}_{7}=\sum_{\substack{G \delta^{-1}<g \leq G^{\prime \prime} \delta^{-1} \\\left(g, p p^{\prime}\right)=1 \\ g \equiv \pi^{\prime}(T)}} e\left(\frac{M \bar{g}}{p p^{\prime}}\right)\left(\frac{g}{v^{\prime}}\right)
$$

and

$$
M \equiv-N v \overline{\left(B_{1} B_{2} \Delta g_{1} \delta\right)}\left(p p^{\prime}\right), \quad \pi^{\prime} \equiv \pi \bar{\delta}(T) .
$$

To estimate $\mathcal{M}_{7}$ we divide it into $\mathcal{O}\left(G_{2}\left(\delta v^{\prime} T p p^{\prime}\right)^{-1}\right)$ complete sums and at most one incomplete sum modulo $v^{\prime} T p p^{\prime}$. We get

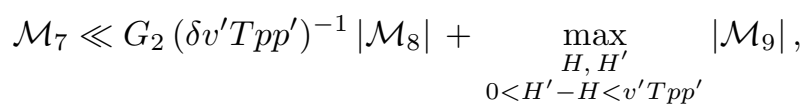


where

$$
\mathcal{M}_{8}=\sum_{\substack{\left.g\left(v^{\prime} T p p^{\prime}\right) \\ g, p p^{\prime}\right)=1 \\ g \equiv \pi^{\prime}(T)}} e\left(\frac{M \bar{g}}{p p^{\prime}}\right)\left(\frac{g}{v^{\prime}}\right), \quad \mathcal{M}_{9}=\sum_{\substack{H<g \leq H^{\prime} \\\left(g, p p^{\prime}\right)=1 \\ g \equiv \pi^{\prime}(T)}} e\left(\frac{M \bar{g}}{p p^{\prime}}\right)\left(\frac{g}{v^{\prime}}\right) .
$$

Consider the complete sum $\mathcal{M}_{8}$. First we note that the relevant values of $g$ are all coprime to $v^{\prime} T p p^{\prime}$. Obviously $\left(v^{\prime} T, p p^{\prime}\right)=1$. Hence we have

$$
\begin{aligned}
\mathcal{M}_{8} & =\sum_{\substack{h_{1}\left(v^{\prime} T\right)^{*}, h_{2}\left(p p^{\prime}\right)^{*} \\
h_{1} p p^{\prime}+h_{2} v^{\prime} T \equiv \pi^{\prime}(T)}} e\left(\frac{M \overline{\left(h_{1} p p^{\prime}+h_{2} v^{\prime} T\right)}}{p p^{\prime}}\right)\left(\frac{h_{1} p p^{\prime}+h_{2} v^{\prime} T}{v^{\prime}}\right) \\
& =\sum_{\substack{h_{1}\left(v^{\prime} T\right)^{*} \\
h_{1} p p^{\prime} \equiv \pi^{\prime}(T)}}\left(\frac{h_{1} p p^{\prime}}{v^{\prime}}\right) c_{p p^{\prime}}\left(M \overline{v^{\prime} T}\right),
\end{aligned}
$$

where $c_{p p^{\prime}}$ is the Ramanujan sum, defined by (4). To estimate it we apply Lemma 5 . We also note that the sum over $h_{1}$ has $\mathcal{O}\left(v^{\prime}\right)$ terms. Therefore we obtain

$$
\mathcal{M}_{8} \ll v^{\prime} .
$$

Consider now the incomplete sum $\mathcal{M}_{9}$. We treat it in the following standard way:

$$
\begin{aligned}
\mathcal{M}_{9} & =\sum_{\substack{g\left(v^{\prime} T p p^{\prime}\right) \\
\left(g, p p^{\prime}\right)=1 \\
g \equiv \pi^{\prime}(T)}} e\left(\frac{M \bar{g}}{p p^{\prime}}\right)\left(\frac{g}{v^{\prime}}\right) \sum_{H<s \leq H^{\prime}}\left(v^{\prime} T p p^{\prime}\right)^{-1} \sum_{h\left(v^{\prime} T p p^{\prime}\right)} e\left(\frac{h(g-s)}{v^{\prime} T p p^{\prime}}\right) \\
& =\left(v^{\prime} T p p^{\prime}\right)^{-1} \sum_{h\left(v^{\prime} T p p^{\prime}\right)}\left(\sum_{H<s \leq H^{\prime}} e\left(\frac{-h s}{v^{\prime} T p p^{\prime}}\right)\right) \mathcal{M}_{10}(h),
\end{aligned}
$$

where

$$
\mathcal{M}_{10}(h)=\sum_{\substack{g\left(v^{\prime} T p p^{\prime}\right) \\\left(g, p p^{\prime}\right)=1 \\ g \equiv \pi^{\prime}(T)}}\left(\frac{g}{v^{\prime}}\right) e\left(\frac{M \bar{g}}{p p^{\prime}}+\frac{h g}{v^{\prime} T p p^{\prime}}\right) .
$$

It is clear that

$$
\mathcal{M}_{9} \ll \sum_{|h| \leq v^{\prime} T p p^{\prime}}(1+|h|)^{-1}\left|\mathcal{M}_{10}(h)\right| .
$$

It remains to estimate $\mathcal{M}_{10}(h)$. Again we note that the relevant values of $g$ are 
all coprime to $v^{\prime} T p p^{\prime}$ and that $\left(v^{\prime} T, p p^{\prime}\right)=1$. Hence we have

$$
\begin{aligned}
\mathcal{M}_{10}(h)= & \sum_{\substack{t_{1}\left(v^{\prime} T\right)^{*}, t_{2}\left(p p^{\prime}\right) \\
t_{1} p p^{\prime}+t_{2} v^{\prime} T \equiv \pi^{\prime}(T)}}\left(\frac{t_{1} p p^{\prime}+t_{2} v^{\prime} T}{v^{\prime}}\right) e\left(\frac{M \overline{\left(v^{\prime} T t_{2}\right)}}{p p^{\prime}}+\frac{t_{1} h}{v^{\prime} T}+\frac{t_{2} h}{p p^{\prime}}\right) \\
= & \sum_{\substack{t_{1}\left(v^{\prime} T\right)^{*} \\
t_{1} p p^{\prime} \equiv \pi^{\prime}(T)}}\left(\frac{t_{1} p p^{\prime}}{v^{\prime}}\right) e\left(\frac{t_{1} h}{v^{\prime} T}\right) K\left(p p^{\prime}, h, M \overline{v^{\prime} T}\right),
\end{aligned}
$$

where $K$ is the Kloosterman sum, defined by (4). We estimate it using Lemma 4 . Obviously, the sum over $t_{1}$ has $\mathcal{O}\left(v^{\prime}\right)$ terms. Hence

$$
\mathcal{M}_{10}(h) \ll v^{\prime} R \text {. }
$$

Inequalities (240) and (241) imply

$$
\mathcal{M}_{9} \ll v^{\prime} R P^{\varepsilon} .
$$

From (238), (239) and (242) we get

$$
\mathcal{M}_{7} \ll\left(G_{2}\left(\delta T R^{2}\right)^{-1}+v^{\prime} R\right) P^{\varepsilon} .
$$

We take into account (231) - (237) and (243) to find that

$$
\mathcal{M}_{1} \ll\left(\left(G_{2}^{1 / 2} \Delta B_{1} B_{2} R^{2}\right)^{-1}+|v| R G_{2}^{-3 / 2}\right) P^{3 \varepsilon} .
$$

To estimate $E\left(G_{1}, G_{2}\right)$ we use (228), (230) and (244). We get

$$
E\left(G_{1}, G_{2}\right) \ll E^{(1)}+E^{(2)},
$$

where $E^{(j)}, j=1,2$, are the contributions to $E\left(G_{1}, G_{2}\right)$ coming from the first, respectively, the second summand from the right hand side of (244). We use (219), (228), (230) and (244) to find that

$$
E^{(1)} \ll P^{3 \varepsilon} R^{-2} E^{\#}\left(G_{1}, G_{2}\right) .
$$

Now we apply (225) to get

$$
E^{(1)} \ll G_{1} G_{2} R^{3} D^{6} Q P^{15 \varepsilon} .
$$

Consider $E^{(2)}$. We use (228), (230) and (244) to get

$$
E^{(2)} \ll P^{4+3 \varepsilon} R^{2} G_{1}^{-1 / 2} G_{2}^{-3 / 2} \sum_{(\mathcal{D})} d_{1} d_{2} d_{3} \mathcal{T}^{*}
$$

where

$$
\mathcal{T}^{*}=\sum_{\substack{\Delta \leq \leq 4 R^{2} Q^{2} P^{\varepsilon-2} \\ \Delta, v>0}} \Delta^{-1} v \sum_{B_{1}, B_{2}(221)}\left(B_{1} B_{2}\right)^{-1 / 2}
$$


It is clear by analogy with (222) that

$$
\mathcal{T}^{*} \ll \sum_{\substack{\Delta v \leq 4 R^{2} Q^{2} P^{\varepsilon-2} \\ \Delta, v>0}} \Delta^{-1} v\left(v R^{-2}\left(\Delta G_{1} G_{2}\right)^{-1} P^{2-\varepsilon}\right)^{-1 / 2} \mathcal{T}_{1}^{2},
$$

where $\mathcal{T}_{1}$ is defined by (223). Using (224), (248) and (249) we easily find that

$$
E^{(2)} \ll R^{6} Q^{3} G_{2}^{-1} D^{6} P^{10 \varepsilon} .
$$

From (227), (245), (247) and (250) we find

$$
E\left(G_{1}, G_{2}\right) \ll\left(G_{1} G_{2} R^{3} Q+R^{6} Q^{3}\left(\max \left(G_{1}, G_{2}\right)\right)^{-1}\right) D^{6} P^{15 \varepsilon} .
$$

Conclusion. Let $H$ be a parameter which we choose below. If $G_{1}, G_{2} \leq H$ then we use the first estimate (226) for $E\left(G_{1}, G_{2}\right)$. If $H<\max \left(G_{1}, G_{2}\right) \leq Q$ then we use the second estimate, given by (251). We obtain

$$
E\left(G_{1}, G_{2}\right) \ll\left(R^{5} H^{2} Q+R^{3} Q^{3}+R^{6} H^{-1} Q^{3}\right) D^{6} P^{15 \varepsilon} .
$$

We choose $H=R^{1 / 3} Q^{2 / 3}$ and use (208) and (252) to obtain

$$
E^{*} \ll\left(R^{3} Q^{3}+R^{17 / 3} Q^{7 / 3}\right) D^{6} P^{16 \varepsilon} .
$$

\subsubsection{The estimate for $\mathcal{E}_{1}$.}

The terms $\mathcal{E}_{1}^{(i)}, i=1,2,3$ from the expression (84) for $\mathcal{E}_{1}$ satisfy the asymptotic formulas (109), (111) and (121), respectively. The quantities $\mathcal{V}^{*}, D^{*}$ and $E^{*}$, included in the $\mathcal{O}$ - terms of these formulas satisfy (193), (194) and (253). Therefore we obtain

$$
\begin{aligned}
\mathcal{E}_{1} \ll\left(P^{3} D^{6} R+P^{2} D^{6} R^{5}\right. & \\
& \left.+P^{4} Q^{-1} D^{3} R+Q^{3} D^{6} R^{3}+Q^{7 / 3} D^{6} R^{17 / 3}\right) P^{20 \varepsilon} .
\end{aligned}
$$

\subsection{Proof of Proposition 1.}

Consider the sum $\mathcal{E}(D, Q)$, defined by (31). From (41) and (43) we get

$$
\mathcal{E}(D, Q) \ll\left(P R^{-2}\left|\mathcal{E}_{1}\right|+P R^{-1} \mathcal{E}_{2}\right)^{1 / 2} P^{\varepsilon} .
$$

To estimate $\mathcal{E}_{2}$ we use (82):

$$
\mathcal{E}_{2} \ll\left(P^{3} D^{6}+P^{4} D^{3} Q^{-1}\right) P^{9 \varepsilon} .
$$

Similarly, for $\mathcal{E}_{1}$ we use the estimate (254), given above. We obtain

$$
\begin{array}{r}
\mathcal{E}(D, Q) \ll\left(P^{2} D^{3} R^{-1 / 2}+P^{3 / 2} D^{3} R^{3 / 2}+P^{5 / 2} Q^{-1 / 2} D^{3 / 2} R^{-1 / 2}\right. \\
\left.+P^{1 / 2} Q^{3 / 2} D^{3} R^{1 / 2}+P^{1 / 2} Q^{7 / 6} D^{3} R^{11 / 6}\right) P^{12 \varepsilon}
\end{array}
$$


The parameters $D, Q, R$ satisfy the conditions (39), (40), (42), (83) and (196):

$$
\begin{aligned}
Q & \leq P^{1-\varepsilon}, \\
D & =P^{\alpha_{0}} \quad \text { where } \quad \alpha_{0} \in(0,1), \\
R & =P^{\alpha_{1}} \quad \text { where } \quad \alpha_{1} \in(0,1), \\
D & \leq R^{1 / 6} P^{-10 \varepsilon}, \\
P^{1+20 \varepsilon} D^{3} R^{-1} & \leq Q, \\
Q & \leq P^{1-\varepsilon} R^{-1 / 2} .
\end{aligned}
$$

It is not difficult to see that the optimal choice (up to a power of $P^{\varepsilon}$ ) of these parameters is

$$
R=P^{5 / 23}, \quad Q=P^{20 / 23}, \quad D=P^{2 / 69-10 \varepsilon} .
$$

From (255) and (256) we obtain

$$
\mathcal{E}(D, Q) \ll P^{2-\varepsilon} .
$$

This completes the proof of Proposition 1.

\subsection{Proof of Proposition 2.}

\subsubsection{Beginning.}

Let us write $\beta(\mathbf{d})=\beta_{1}\left(d_{1}\right) \beta_{2}\left(d_{2}\right) \beta_{3}\left(d_{3}\right)$ for brevity and consider the sum

$$
\mathcal{H}_{1}=\sum_{(\mathcal{D})} \beta(\mathbf{d}) \mathcal{L}_{\mathbf{d}}(N) .
$$

We use (28), (30), (34) and Proposition 1 to get

$$
\mathcal{H}_{1}=\sum_{(\mathcal{D})} \beta(\mathbf{d}) \sum_{p \leq P} \Omega_{\mathbf{d}}\left(N-p^{2}\right)=\mathcal{H}_{2}+\mathcal{O}\left(P^{2-\varepsilon}\right),
$$

where

$$
\mathcal{H}_{2}=\sum_{(\mathcal{D})} \beta(\mathbf{d}) \sum_{p \leq P} \mathcal{M}_{\mathbf{d}, Q}\left(N-p^{2}\right) .
$$

Here $\mathcal{M}_{\mathbf{d}, Q}$ is defined by (29) and $Q$ satisfies (32). We use (29) and change the order of summation to find that

$$
\begin{aligned}
\mathcal{H}_{2}=P \sum_{(\mathcal{D})} \frac{\beta(\mathbf{d})}{d_{1} d_{2} d_{3}} \sum_{q \leq Q} q^{-3} \sum_{a(q)^{*}} S_{\mathbf{d}}(q, a) \\
\times \sum_{t_{0} P<p \leq P} H\left(1-\frac{p^{2}}{N}\right) e_{q}\left(a\left(p^{2}-N\right)\right),
\end{aligned}
$$


where $t_{0}=\left(1-t_{2}\right)^{1 / 2} \in(0,1)$ and $t_{2}$ is specified in Lemma 11 . Now we apply Abel's formula to get

$$
\mathcal{H}_{2}=-P \int_{t_{0} P}^{P} \mathcal{B}(x) \frac{d}{d x}\left(H\left(1-\frac{x^{2}}{N}\right)\right) d x,
$$

where

$$
\mathcal{B}(x)=\sum_{(\mathcal{D})} \frac{\beta(\mathbf{d})}{d_{1} d_{2} d_{3}} \sum_{q \leq Q} q^{-3} \sum_{a(q)^{*}} S_{\mathbf{d}}(q, a) \mathcal{Z}(x)
$$

and

$$
\mathcal{Z}(x)=\mathcal{Z}=\sum_{t_{0} P<p \leq x} e_{q}\left(a\left(p^{2}-N\right)\right) .
$$

Consider the $\operatorname{sum} \mathcal{Z}$. We divide it into subsums according to the congruence class $m$ of $p$ modulo $q$. There is no contribution from $m$ such that $(m, q)>1$. Therefore we get

$$
\begin{aligned}
\mathcal{Z}= & \sum_{m(q)^{*}} e_{q}\left(a\left(m^{2}-N\right)\right)\left(\pi(x, q, m)-\pi\left(t_{0} P, q, m\right)\right) \\
= & \frac{1}{\varphi(q)} \int_{t_{0} P}^{x} \frac{d t}{\log t} e_{q}(-a N) T(q, a) \\
& \quad+\sum_{m(q)^{*}} e_{q}\left(a\left(m^{2}-N\right)\right)\left(\Delta(x, q, m)-\Delta\left(t_{0} P, q, m\right)\right)
\end{aligned}
$$

where $\Delta(x, q, m)$ and $T(q, a)$ are defined by (3) and (7), respectively.

From (262) - (264) we get

$$
\mathcal{B}(x)=\mathcal{B}_{0} \int_{t_{0} P}^{x} \frac{d t}{\log t}+\mathcal{C}(x)-\mathcal{C}\left(t_{0} P\right),
$$

where

$$
\begin{aligned}
& \mathcal{C}(x)= \sum_{(\mathcal{D})} \frac{\beta(\mathbf{d})}{d_{1} d_{2} d_{3}} \sum_{q \leq Q} q^{-3} \\
& \times \sum_{a(q)^{*}} S_{\mathbf{d}}(q, a) \sum_{m(q)^{*}} e_{q}\left(a\left(m^{2}-N\right)\right) \Delta(x, q, m), \\
& \mathcal{B}_{0}=\sum_{(\mathcal{D})} \frac{\beta(\mathbf{d})}{d_{1} d_{2} d_{3}} \sum_{q \leq Q} h_{\mathbf{d}}(q)
\end{aligned}
$$

and where $h_{\mathbf{d}}(q)$ is defined by (9). 


\subsubsection{The estimation of $\mathcal{C}(x)$.}

Consider the sum $\mathcal{C}(x)$. We expect that it is negligible because it depends on the quantity $\Delta(x, q, m)$, which is small on average with respect to $q$ and $m$. More precisely, we shall prove that if $t_{0} P \leq x \leq P$ then we have

$$
\mathcal{C}(x) \ll P(\log P)^{-A} .
$$

It is clear that

$$
\mathcal{C}(x)=\sum_{q \leq Q} \sum_{m(q)^{*}} \Gamma(q, m) \Delta(x, q, m)
$$

where

$$
\Gamma(q, m)=q^{-3} \sum_{(\mathcal{D})} \frac{\beta(\mathbf{d})}{d_{1} d_{2} d_{3}} \zeta_{\mathbf{d}}(q, m)
$$

and

$$
\zeta_{\mathbf{d}}(q, m)=\sum_{a(q)^{*}} S_{\mathbf{d}}(q, a) e_{q}\left(a\left(m^{2}-N\right)\right) .
$$

Obviously, $\Gamma(q, m)$ is always real. Using Cauchy's inequality we get

$$
|\mathcal{C}(x)|^{2} \leq L M
$$

where

$$
\begin{aligned}
L & =\sum_{q \leq Q} \sum_{m(q)^{*}} \Delta(x, q, m)^{2}, \\
M & =\sum_{q \leq Q} \sum_{m(q)^{*}} \Gamma(q, m)^{2} .
\end{aligned}
$$

We estimate $L$ using Lemma 1 . Consider the sum $M$. We shall prove that

$$
M \ll(\log P)^{C}
$$

for some absolute constant $C>0$. Then we will take into account (271) and the proof of (268) will be complete.

From (269) and (270) we get

$$
M=\sum_{\substack{d_{1}, d_{2}, d_{3} \leq D \\ h_{1}, h_{2}, h_{3} \leq D}} \frac{\beta(\mathbf{d}) \beta(\mathbf{h})}{d_{1} d_{2} d_{3} h_{1} h_{2} h_{3}} \sum_{q \leq Q} \lambda(q, \mathbf{d}, \mathbf{h}),
$$

where

$$
\lambda(q, \mathbf{d}, \mathbf{h})=q^{-6} \sum_{m(q)^{*}} \zeta_{\mathbf{d}}(q, m) \zeta_{\mathbf{h}}(q, m)
$$


Applying Lemma $2(i)$ we easily see that the function $\lambda$ is multiplicative with respect to $q$.

From (35) we conclude that the relevant values of $\mathbf{d}$ and $\mathbf{h}$ are vectors with odd components. Furthermore, we use (270), (276) and Lemma $2(v i)$ and find that

$$
\lambda\left(2^{l}, \mathbf{d}, \mathbf{h}\right) \ll 1 .
$$

Consider $\lambda\left(p^{l}, \mathbf{d}, \mathbf{h}\right)$, where $p>2$ is a prime. Assume that

$$
p^{\alpha_{i}}\left\|d_{i}, \quad p^{\beta_{i}}\right\| h_{i}, \quad \nu_{i}=\min \left(l, 2 \alpha_{i}\right), \quad \mu_{i}=\min \left(l, 2 \beta_{i}\right) .
$$

Using (4) and Lemma $2(i i)$ and (iv) we get

$$
\zeta_{\mathbf{d}}\left(p^{l}, m\right)=\zeta_{\mathbf{d}}^{*}\left(p^{l}, m\right) \prod_{i=1}^{3}\left(p^{\nu_{i}} S\left(p^{l-\nu_{i}}, 1\right)\right)
$$

where

(280) $\zeta_{\mathbf{d}}^{*}\left(p^{l}, m\right)=\left\{\begin{array}{lll}c_{p^{l}}\left(m^{2}-N\right) & \text { if } & \nu_{1}+\nu_{2}+\nu_{3} \equiv l(2), \\ \sum_{a\left(p^{l}\right)^{*}}\left(\frac{a}{p}\right) e_{p^{l}}\left(a\left(m^{2}-N\right)\right) & \text { if } & \nu_{1}+\nu_{2}+\nu_{3} \neq \equiv l(2) .\end{array}\right.$

From (276), (278) - (280) and Lemma $2(v)$ we obtain

$$
\begin{aligned}
\left|\lambda\left(p^{l}, \mathbf{d}, \mathbf{h}\right)\right| & \leq p^{-6 l}\left(\prod_{i=1}^{3} p^{l+\frac{1}{2}\left(\nu_{i}+\mu_{i}\right)}\right) \sum_{m\left(p^{l}\right)^{*}}\left|\zeta_{\mathbf{d}}^{*}\left(p^{l}, m\right) \zeta_{\mathbf{h}}^{*}\left(p^{l}, m\right)\right| \\
& \leq p^{-3 l+\frac{1}{2}\left(\nu_{1}+\nu_{2}+\nu_{3}+\mu_{1}+\mu_{2}+\mu_{3}\right)}\left(T_{1}+T_{2}\right),
\end{aligned}
$$

where

$$
\begin{aligned}
T_{1} & =\sum_{m\left(p^{l}\right)^{*}}\left|c_{p^{l}}\left(m^{2}-N\right)\right|^{2}, \\
T_{2} & =\sum_{m\left(p^{l}\right)^{*}}\left|\sum_{a\left(p^{l}\right)^{*}}\left(\frac{a}{p}\right) e_{p^{l}}\left(a\left(m^{2}-N\right)\right)\right|^{2} .
\end{aligned}
$$

We shall prove that

$$
T_{1} \leq 3 p^{2 l}, \quad T_{2} \leq 3 p^{2 l} .
$$

These inequalities and (281) imply that for any prime $p>2$ and for any integer $l$ we have

$$
\left|\lambda\left(p^{l}, \mathbf{d}, \mathbf{h}\right)\right| \leq 6 p^{-l+\frac{1}{2}\left(\nu_{1}+\nu_{2}+\nu_{3}+\mu_{1}+\mu_{2}+\mu_{3}\right)} .
$$

From (277), (278) and (285) we obtain

$$
\begin{aligned}
\sum_{q \leq Q} \lambda(q, \mathbf{d}, \mathbf{h}) & \ll \sum_{2^{\alpha} \leq Q} \sum_{\substack{m \leq Q \\
(m, 2)=1}} \lambda(m, \mathbf{d}, \mathbf{h}) \\
& \ll(\log P) \sum_{m \leq Q} \frac{6^{\nu(m)}}{m} \prod_{i=1}^{3}\left(m, d_{i}\right)\left(m, h_{i}\right) .
\end{aligned}
$$


Now we use (36), (275) and (286) to find that

$$
M \ll(\log P) \sum_{q \leq Q} \frac{6^{\nu(q)}}{q}\left(\sum_{d \leq D} \frac{\tau(d)(q, d)}{d}\right)^{6} .
$$

It is easy to see that the sum over $d$ is $\ll \tau^{2}(q)(\log P)^{2}$. Hence we get

$$
M \ll(\log P)^{13} \sum_{q \leq Q} \frac{6^{\nu(q)}}{q} \tau^{12}(q) \ll(\log P)^{13} \sum_{q \leq Q} \frac{\tau^{15}(q)}{q} \ll(\log P)^{2^{16}},
$$

so we have established (274).

It remains to prove that if $p>2$ is a prime and $l$ is an integer then the inequalities (284) hold.

Consider $T_{1}$. Using the exact formula for the Ramanujan sum, given by Lemma 5, we get

$$
\begin{aligned}
T_{1} & =\sum_{0 \leq h \leq l} \sum_{\substack{m\left(p^{l}\right)^{*} \\
\left(p^{l}, m^{2}-N\right)=p^{h}}}\left|\frac{\varphi\left(p^{l}\right)}{\varphi\left(p^{l-h}\right)} \mu\left(p^{l-h}\right)\right|^{2} \\
& \leq p^{2 l-2} \sum_{\substack{m\left(p^{l}\right)^{*} \\
m^{2} \equiv N\left(p^{l-1}\right)}} 1+p^{2 l} H_{N}\left(p^{l}\right) \\
& =p^{2 l-1} H_{N}\left(p^{l-1}\right)+p^{2 l} H_{N}\left(p^{l}\right)
\end{aligned}
$$

where $H_{N}(q)$ is the cardinality of the set $\left\{m(q)^{*} \mid m^{2} \equiv N(q)\right\}$. It is well known that if $p>2$ and $p \nmid N$ then $H_{N}\left(p^{l}\right) \leq 2$ for any $l$. Obviously, if $p \mid N$ then $H_{N}\left(p^{l}\right)=0$. The inequality (284) for $T_{1}$ follows.

Consider $T_{2}$. Using Lemma 6 (ii) we easily obtain

$$
\sum_{a\left(p^{l}\right)^{*}}\left(\frac{a}{p}\right) e_{p^{l}}(a n)= \begin{cases}p^{l-1} S(p, 1)\left(\frac{n / p^{l-1}}{p}\right) & \text { if } p^{l-1} \| n \\ 0 & \text { otherwise }\end{cases}
$$

We apply Lemma $2(v)$ and the estimate for $H_{N}\left(p^{l}\right)$, given above, and find that

$$
\begin{aligned}
T_{2} & \leq \sum_{\substack{m\left(p^{l}\right)^{*} \\
\left(p^{l}, m^{2}-N\right)=p^{l-1}}}\left|p^{l-1} S(p, 1)\right|^{2} \leq p^{2 l-1} \sum_{\substack{m\left(p^{l}\right)^{*} \\
\left(p^{l}, m^{2}-N\right)=p^{l-1}}} 1 \\
& \leq p^{2 l} H_{N}\left(p^{l-1}\right) \leq 2 p^{2 l} .
\end{aligned}
$$

So the inequalities (284) are established and the proof of (268) is complete. 


\subsubsection{The end of the proof of Proposition 2 .}

Let us consider the sum $\mathcal{B}_{0}$, defined by (267). We extend the summation over $q$ to infinity. Using (17) and (32) we find that

$$
\sum_{q>Q}\left|h_{\mathbf{d}}(q)\right| \ll\left(d_{1} d_{2} d_{3}\right)^{3} \sum_{q>Q} \frac{(q, N)}{q^{2-\varepsilon}} \ll D^{9} Q^{-1} P^{2 \varepsilon} \ll P^{-1 / 5} .
$$

Hence

$$
\mathcal{B}_{0}=\mathcal{B}_{1}+\mathcal{O}\left(P^{-\varepsilon}\right),
$$

where

$$
\mathcal{B}_{1}=\sum_{(\mathcal{D})} \frac{\beta(\mathbf{d})}{d_{1} d_{2} d_{3}} \Sigma_{0}
$$

and where $\Sigma_{0}$ is defined by (16). From (265), (268) and (288) we find that if $t_{0} P \leq x \leq P$ then

$$
\mathcal{B}(x)=\mathcal{B}_{1} \int_{t_{0} P}^{x} \frac{d t}{\log t}+\mathcal{O}\left(P(\log P)^{-A}\right) .
$$

We substitute this expression for $\mathcal{B}(x)$ in (261). The contribution to $\mathcal{H}_{2}$ coming from the remainder term is

$$
\ll P^{2}(\log P)^{-A} \int_{t_{0} P}^{P}\left|H^{\prime}\left(1-\frac{x^{2}}{P^{2}}\right) \frac{2 x}{P^{2}}\right| d x \ll P^{2}(\log P)^{-A} .
$$

We integrate by parts and find that the contribution to $\mathcal{H}_{2}$ from the main term of (290) is $\mathcal{N}_{0} \mathcal{B}_{1}$, where $\mathcal{N}_{0}$ is defined by (26). Now we apply (259) to find that

$$
\mathcal{H}_{1}=\mathcal{N}_{0} \mathcal{B}_{1}+\mathcal{O}\left(P^{2}(\log P)^{-A}\right) .
$$

We take into account (37), (258), (289) and (291) and the proof of Proposition 2 is complete.

\section{The proof of Theorem 1.}

Consider the sum

$$
\mathfrak{F}=\sum_{\substack{p^{2}+x_{1}^{2}+x_{2}^{2}+x_{3}^{2}=N \\\left(x_{i}, \mathfrak{P}\right)=1}} \omega(\mathbf{x}),
$$

where

$$
\mathfrak{P}=\prod_{2<p<z_{1}} p \quad \text { and } \quad z_{1}=P^{\alpha}
$$


for some $\alpha \in(0,1)$, which we shall specify later. Using the condition $N \equiv 4(24)$ and the definition of $\omega(\mathbf{x})$ we find that the solutions of (2), such that $2 \mid p x_{1} x_{2} x_{3}$, are not counted in $\mathfrak{F}$.

Suppose that

$$
\mathfrak{F} \gg P^{2}(\log P)^{-4}
$$

Then there exist constants $c_{0}>0$ and $N_{0}>0$ such that for any integer $N>N_{0}$ there are at least $c_{0} P^{2}(\log P)^{-4}$ quadruples $p, x_{1}, x_{2}, x_{3}$ satisfying $(2)$ and such that $p$ is a prime and each $x_{i}$ is an almost-prime with no more than $\alpha^{-1}$ prime factors, counted according to multiplicity.

So, our aim is to establish (293) with $\alpha$ as large as possible. We apply the vector sieve proposed by Iwaniec [12] and used also by Brüdern and Fouvry [1], [2] as well as by the second author [19], [20]. In many places we omit the calculations because they are similar to those in [1], [19] and [20].

It is convenient to sieve by the small primes separately. Define

$$
z_{0}=(\log P)^{1000}, \quad \mathfrak{P}_{0}=\prod_{2<p<z_{0}} p, \quad \mathfrak{P}_{1}=\prod_{z_{0} \leq p<z_{1}} p
$$

We represent the sum $\mathfrak{F}$ as

$$
\mathfrak{F}=\sum_{p^{2}+x_{1}^{2}+x_{2}^{2}+x_{3}^{2}=N} \omega(\mathbf{x}) \Phi_{1} \Phi_{2} \Phi_{3} \Lambda_{1} \Lambda_{2} \Lambda_{3},
$$

where

$$
\Phi_{i}=\sum_{k \mid\left(x_{i}, \mathfrak{P}_{0}\right)} \mu(k), \quad \Lambda_{i}=\sum_{l \mid\left(x_{i}, \mathfrak{P}_{1}\right)} \mu(l)
$$

Let

$$
D_{0}=P^{\varepsilon}, \quad D_{1}=P^{2 / 69-11 \varepsilon}, \quad D=D_{0} D_{1}
$$

We define

$$
\begin{aligned}
& s_{0}=\frac{\log D_{0}}{\log z_{0}}=\frac{\varepsilon \log P}{1000 \log \log P}, \\
& s_{1}=\frac{\log D_{1}}{\log z_{1}}=\left(\frac{2}{69}-11 \varepsilon\right) \alpha^{-1} .
\end{aligned}
$$

To apply the sieve method we need the inequalities $s_{0}>2$ and $s_{1}>2$. The first of them is obvious. To have the second we assume that $\alpha<1 / 69$ and take $\varepsilon$ suffisiently small.

Consider Rosser's weights $\lambda_{i}^{ \pm}(d)$ of orders $D_{i}, i=0,1$. Define

$$
\Phi_{i}^{ \pm}=\sum_{k \mid\left(x_{i}, \mathfrak{P}_{0}\right)} \lambda_{0}^{ \pm}(k), \quad \Lambda_{i}^{ \pm}=\sum_{l \mid\left(x_{i}, \mathfrak{P}_{1}\right)} \lambda_{1}^{ \pm}(l), \quad i=1,2,3 .
$$


The definition and the properties of the Rosser weights can be found in Iwaniec [13], [14]. In particular, we have

$$
\begin{array}{lc}
\left|\lambda_{i}^{ \pm}(d)\right| \leq 1 ; & \lambda_{i}^{ \pm}(d)=0 \quad \text { if } \quad \mu(d)=0 \quad \text { or } \quad d>D_{i} ; \\
\Phi_{i}^{-} \leq \Phi_{i} \leq \Phi_{i}^{+}, \quad \Lambda_{i}^{-} \leq \Lambda_{i} \leq \Lambda_{i}^{+}, \quad i=1,2,3 .
\end{array}
$$

From (301) we easily get

$$
\begin{aligned}
& \Phi_{1} \Phi_{2} \Phi_{3} \Lambda_{1} \Lambda_{2} \Lambda_{3} \geq \Phi_{1}^{-} \Phi_{2}^{+} \Phi_{3}^{+} \Lambda_{1}^{+} \Lambda_{2}^{+} \Lambda_{3}^{+}+\Phi_{1}^{+} \Phi_{2}^{-} \Phi_{3}^{+} \Lambda_{1}^{+} \Lambda_{2}^{+} \Lambda_{3}^{+} \\
& \quad+\Phi_{1}^{+} \Phi_{2}^{+} \Phi_{3}^{-} \Lambda_{1}^{+} \Lambda_{2}^{+} \Lambda_{3}^{+}+\Phi_{1}^{+} \Phi_{2}^{+} \Phi_{3}^{+} \Lambda_{1}^{-} \Lambda_{2}^{+} \Lambda_{3}^{+}+\Phi_{1}^{+} \Phi_{2}^{+} \Phi_{3}^{+} \Lambda_{1}^{+} \Lambda_{2}^{-} \Lambda_{3}^{+} \\
& \quad+\Phi_{1}^{+} \Phi_{2}^{+} \Phi_{3}^{+} \Lambda_{1}^{+} \Lambda_{2}^{+} \Lambda_{3}^{-}-5 \Phi_{1}^{+} \Phi_{2}^{+} \Phi_{3}^{+} \Lambda_{1}^{+} \Lambda_{2}^{+} \Lambda_{3}^{+}
\end{aligned}
$$

The proof of (302) is elementary and similar to the proof of Lemma 13 of [1].

From (295) and (302) we find that

$$
\mathfrak{F} \geq \sum_{i=1}^{6} \mathfrak{F}_{i}-5 \mathfrak{F}_{7},
$$

where $\mathfrak{F}_{i}, 1 \leq i \leq 7$ are the contributions of the consecutive terms from the right hand side of (302). Obviously $\mathfrak{F}_{1}=\mathfrak{F}_{2}=\mathfrak{F}_{3}$ and $\mathfrak{F}_{4}=\mathfrak{F}_{5}=\mathfrak{F}_{6}$.

Consider, for example, $\mathfrak{F}_{1}$. We use (296), (299), (300) and change the order of summation to get

$$
\mathfrak{F}_{1}=\sum_{d_{1}, d_{2}, d_{3} \leq D} \beta_{1}\left(d_{1}\right) \beta_{2}\left(d_{2}\right) \beta_{3}\left(d_{3}\right) \mathcal{L}_{\mathbf{d}}(N),
$$

where $\mathcal{L}_{\mathbf{d}}(N)$ is defined by (34) and

$$
\begin{aligned}
\beta_{1}\left(d_{1}\right) & =\sum_{\substack{k\left|\mathfrak{P}_{0}, l\right| \mathfrak{\beta}_{1} \\
k l=d_{1}}} \lambda_{0}^{-}(k) \lambda_{1}^{+}(l), \\
\beta_{i}\left(d_{i}\right) & =\sum_{\substack{k\left|\mathfrak{P}_{0}, l\right| \mathfrak{\beta}_{1} \\
k l=d_{i}}} \lambda_{0}^{+}(k) \lambda_{1}^{+}(l), \quad i=2,3 .
\end{aligned}
$$

It is obvious that the functions $\beta_{i}$ satisfy (35) and (36). Therefore, we can apply Proposition 2 to get

$$
\mathfrak{F}_{1}=\mathfrak{F}_{1}^{*}+\mathcal{O}\left(P^{2}(\log P)^{-A}\right),
$$

where

$$
\mathfrak{F}_{1}^{*}=\sum_{d_{1}, d_{2}, d_{3} \leq D} \frac{\beta_{1}\left(d_{1}\right) \beta_{2}\left(d_{2}\right) \beta_{3}\left(d_{3}\right)}{d_{1} d_{2} d_{3}} \mathcal{N}_{0}(N) \Sigma_{0}(\mathbf{d}, N)
$$

and where $\Sigma_{0}$ and $\mathcal{N}_{0}$ are defined by (16) and (26). 
Suppose that $d_{i}$ are squarefree odd numbers and consider $\Sigma_{0}$. Using the identity (16) we find that

$$
\Sigma_{0}(\mathbf{d}, N)=\xi_{0}(N) \mathfrak{R}(\mathbf{d}, N),
$$

where

(310) $\mathfrak{R}(\mathbf{d}, N)=\prod_{p \| d_{1} d_{2} d_{3}} \frac{1+h_{1}(p)}{1+h_{0}(p)} \prod_{p^{2} \| d_{1} d_{2} d_{3}} \frac{1+h_{2}(p)}{1+h_{0}(p)} \prod_{p^{3} \| d_{1} d_{2} d_{3}} \frac{1+h_{3}(p)}{1+h_{0}(p)}$

From (10) - (13), (309) and (310) we get

$$
1 \ll \xi_{0} \ll \log \log P
$$

and

$$
\mathfrak{R}(\mathbf{d}, N) \ll \tau^{2}\left(d_{1}\right) \tau^{2}\left(d_{2}\right) \tau^{2}\left(d_{3}\right) .
$$

The calculations are standard and we leave them to the reader.

Suppose that $d_{i}=k_{i} l_{i}$, where $k_{i}\left|\mathfrak{P}_{0}, l_{i}\right| \mathfrak{P}_{1}, i=1,2,3$, and denote by $\mathbf{k}$ and $\mathbf{l}$ the triples $k_{1}, k_{2}, k_{3}$ and $l_{1}, l_{2}, l_{3}$, respectively. It is clear that

$$
\mathfrak{R}(\mathbf{d}, N)=\mathfrak{R}(\mathbf{k}, N) \mathfrak{R}(\mathbf{l}, N) .
$$

Define

$$
\begin{aligned}
\mathcal{H}^{ \pm} & =\sum_{k_{1}, k_{2}, k_{3} \mid \mathfrak{P}_{0}} \frac{\lambda_{0}^{ \pm}\left(k_{1}\right) \lambda_{0}^{+}\left(k_{2}\right) \lambda_{0}^{+}\left(k_{3}\right)}{k_{1} k_{2} k_{3}} \mathfrak{R}(\mathbf{k}, N) \\
\mathcal{G}^{ \pm} & =\sum_{l_{1}, l_{2}, l_{3} \mid \mathfrak{P}_{1}} \frac{\lambda_{1}^{ \pm}\left(l_{1}\right) \lambda_{1}^{+}\left(l_{2}\right) \lambda_{1}^{+}\left(l_{3}\right)}{l_{1} l_{2} l_{3}} \mathfrak{R}(\mathbf{l}, N) .
\end{aligned}
$$

From (304), (305), (307), (308) and (313) - (315) we find that

$$
\mathfrak{F}_{1}^{*}=\mathcal{N}_{0} \xi_{0} \mathcal{H}^{-} \mathcal{G}^{+} .
$$

We study the other sums $\mathfrak{F}_{i}$ in the same manner and use the analogs of (306) and (316) to obtain

$$
\begin{aligned}
& \mathfrak{F}_{1}=\mathfrak{F}_{2}= \mathfrak{F}_{3}=\mathcal{N}_{0} \xi_{0} \mathcal{H}^{-} \mathcal{G}^{+}+\mathcal{O}\left(P^{2}(\log P)^{-A}\right), \\
& \mathfrak{F}_{4}=\mathfrak{F}_{5}= \mathfrak{F}_{6}=\mathcal{N}_{0} \xi_{0} \mathcal{H}^{+} \mathcal{G}^{-}+\mathcal{O}\left(P^{2}(\log P)^{-A}\right), \\
& \mathfrak{F}_{7}=\mathcal{N}_{0} \xi_{0} \mathcal{H}^{+} \mathcal{G}^{+}+\mathcal{O}\left(P^{2}(\log P)^{-A}\right) .
\end{aligned}
$$

Now we apply (303) to get

$$
\mathfrak{F} \geq \mathcal{N}_{0} \xi_{0}\left(3 \mathcal{H}^{-} \mathcal{G}^{+}+3 \mathcal{H}^{+} \mathcal{G}^{-}-5 \mathcal{H}^{+} \mathcal{G}^{+}\right)+\mathcal{O}\left(P^{2}(\log P)^{-A}\right) .
$$


From (297) we see that $s_{0} \rightarrow \infty$ as $N \rightarrow \infty$. Hence the Rosser weights $\lambda_{0}^{ \pm}$ behave like the Möbius function and we can expect that the sums $\mathcal{H}^{ \pm}$can be approximated by the sum

$$
\mathcal{H}_{0}=\sum_{k_{1}, k_{2}, k_{3} \mid \mathfrak{P}_{0}} \frac{\mu\left(k_{1}\right) \mu\left(k_{2}\right) \mu\left(k_{3}\right)}{k_{1} k_{2} k_{3}} \mathfrak{R}(\mathbf{k}, N) .
$$

More precisely, the following asymptotic formula holds:

$$
\mathcal{H}^{ \pm}=\mathcal{H}_{0}+\mathcal{O}(\exp (-\sqrt{\log P})) .
$$

We omit the proof because it does not differ significantly from the proof of Lemma 14 of [19] or formula (3.17) of [20].

It is easy to see that

$$
\mathcal{H}_{0}=\prod_{2<p<z_{0}}\left(1-\frac{3\left(1+h_{1}(p)\right)}{p\left(1+h_{0}(p)\right)}+\frac{3\left(1+h_{2}(p)\right)}{p^{2}\left(1+h_{0}(p)\right)}-\frac{1+h_{3}(p)}{p^{3}\left(1+h_{0}(p)\right)}\right) .
$$

From this formula, (10) - (13) and (294) we find that

$$
\mathcal{H}_{0} \asymp(\log \log P)^{-3} .
$$

We leave the easy verification of (319) and (320) to the reader.

Using (292), (294), (300), (312) and (315) we get

$$
\mathcal{G}^{ \pm} \ll\left(\sum_{l \mid \mathfrak{P}_{1}} \frac{\tau^{2}(l)}{l}\right)^{3} \ll(\log P)^{12}
$$

From (27), (311), (317), (318) and (321) we find that

$$
\mathfrak{F} \geq \mathcal{N}_{0} \xi_{0} \mathcal{H}_{0}\left(3 \mathcal{G}^{-}-2 \mathcal{G}^{+}\right)+\mathcal{O}\left(P^{2}(\log P)^{-A}\right) .
$$

It remains to estimate from below the difference $3 \mathcal{G}^{-}-2 \mathcal{G}^{+}$. Using $(315)$ we get

$$
\begin{aligned}
3 \mathcal{G}^{-}-2 \mathcal{G}^{+} & =\sum_{l_{1}, l_{2}, l_{3} \mid \mathfrak{P}_{1}} \frac{\left(3 \lambda_{1}^{-}\left(l_{1}\right)-2 \lambda_{1}^{+}\left(l_{1}\right)\right) \lambda_{1}^{+}\left(l_{2}\right) \lambda_{1}^{+}\left(l_{3}\right)}{l_{1} l_{2} l_{3}} \mathfrak{R}(\mathbf{l}, N) \\
& =W_{1}+W_{1}^{\prime}
\end{aligned}
$$

where in $W_{1}$ we sum over $l_{1}, l_{2}, l_{3} \mid \mathfrak{P}_{1}$ such that

$$
\left(l_{1}, l_{2}\right)=\left(l_{1}, l_{3}\right)=\left(l_{2}, l_{3}\right)=1
$$

and where $W_{1}^{\prime}$ is the contribution of the other terms. We use the definition of $\mathfrak{P}_{1}$, given by $(294)$, and find that if $l_{i}, l_{j} \mid \mathfrak{P}_{1}$ and $\left(l_{i}, l_{j}\right)>1$ then $\left(l_{i}, l_{j}\right) \geq z_{0}$. From this observation, (300) and (312) we easily get

$$
W_{1}^{\prime} \ll(\log P)^{100} z_{0}^{-1} .
$$


Consider $W_{1}$. If the condition (324) holds then we have

$$
\mathfrak{R}(\mathbf{l}, N)=\psi\left(l_{1}\right) \psi\left(l_{2}\right) \psi\left(l_{3}\right),
$$

where

$$
\psi(l)=\prod_{\substack{p \mid l \\ p>2}} \frac{1+h_{1}(p)}{1+h_{0}(p)} .
$$

Hence

$$
\begin{aligned}
& \text { (327) } W_{1}=\sum_{l_{1}, l_{2}, l_{3} \mid \mathfrak{P}_{1}} \frac{\left(3 \lambda_{1}^{-}\left(l_{1}\right)-2 \lambda_{1}^{+}\left(l_{1}\right)\right) \lambda_{1}^{+}\left(l_{2}\right) \lambda_{1}^{+}\left(l_{3}\right)}{l_{1} l_{2} l_{3}} \psi\left(l_{1}\right) \psi\left(l_{2}\right) \psi\left(l_{3}\right) \\
& \times \sum_{\substack{h_{1}\left|\left(l_{2}, l_{3}\right) \\
h_{2}\right|\left(l_{1}, l_{3}\right) \\
h_{3} \mid\left(l_{1}, l_{2}\right)}} \mu\left(h_{1}\right) \mu\left(h_{2}\right) \mu\left(h_{3}\right) \\
& =\sum_{h_{1}, h_{2}, h_{3} \mid \mathfrak{P}_{1}} \mu\left(h_{1}\right) \mu\left(h_{2}\right) \mu\left(h_{3}\right) \\
& \times \sum_{\substack{l_{1}, l_{2}, l_{3} \mid \mathfrak{P}_{1} \\
l_{1} \equiv 0\left(\left[h_{2}, h_{3}\right]\right) \\
l_{2} \equiv 0\left(\left[h_{1}, h_{3}\right]\right) \\
l_{3} \equiv 0\left(\left[h_{1}, h_{2}\right]\right)}} \frac{\left(3 \lambda_{1}^{-}\left(l_{1}\right)-2 \lambda_{1}^{+}\left(l_{1}\right)\right) \lambda_{1}^{+}\left(l_{2}\right) \lambda_{1}^{+}\left(l_{3}\right)}{l_{1} l_{2} l_{3}} \psi\left(l_{1}\right) \psi\left(l_{2}\right) \psi\left(l_{3}\right) \\
& =W_{2}+W_{2}^{\prime} \text {, }
\end{aligned}
$$

where $W_{2}$ is the contribution of the terms with $h_{1}=h_{2}=h_{3}=1$ and $W_{2}^{\prime}$ comes from the other terms.

Consider $W_{2}^{\prime}$. If $h_{i} \mid \mathfrak{P}_{1}$ and $h_{i}>1$ then $h_{i} \geq z_{0}$. Therefore, after some calculations, which we leave to the reader, we obtain

$$
W_{2}^{\prime} \ll(\log P)^{100} z_{0}^{-1}
$$

Consider $W_{2}$. Obviously

$$
W_{2}=\left(3 \mathfrak{T}^{-}-2 \mathfrak{T}^{+}\right)\left(\mathfrak{T}^{+}\right)^{2},
$$

where

$$
\mathfrak{T}^{ \pm}=\sum_{l \mid \mathfrak{P}_{1}} \frac{\lambda_{1}^{ \pm}(l) \psi(l)}{l} .
$$

Using Lemma 10 of [1] we establish the inequalities

$$
\begin{aligned}
\mathfrak{N} \leq \mathfrak{T}^{+} & \leq \mathfrak{N}\left\{F\left(s_{1}\right)+\mathcal{O}\left((\log P)^{-1 / 3}\right)\right\} \\
\mathfrak{T}^{-} & \geq \mathfrak{N}\left\{f\left(s_{1}\right)+\mathcal{O}\left((\log P)^{-1 / 3}\right)\right\}
\end{aligned}
$$


where $F$ and $f$ are the functions of the linear sieve (see Iwaniec [13], [14]), $s_{1}$ is specified by (298),

$$
\mathfrak{N}=\prod_{z_{0} \leq p<z_{1}}\left(1-\frac{\psi(p)}{p}\right)
$$

and $\psi$ is defined by (326). We use (10), (11), (292), (294) and (326) to find that

$$
\mathfrak{N} \gg \frac{\log z_{0}}{\log z_{1}} \gg(\log P)^{-1}(\log \log P) .
$$

We choose $\alpha=0.00983$ and use that if $\gamma$ denotes the Euler constant and $s \in(2,3)$ then $F(s)=2 e^{\gamma} s^{-1}$ and $f(s)=2 e^{\gamma} s^{-1} \log (s-1)$. We find that if $s_{1}$ is specified by (298) and $\varepsilon$ is sufficiently small then

$$
3 f\left(s_{1}\right)-2 F\left(s_{1}\right)>10^{-4} .
$$

From (329) - (333) we obtain

(334) $W_{2} \geq \mathfrak{N}^{3}\left(3 f\left(s_{1}\right)-2 F\left(s_{1}\right)+\mathcal{O}\left((\log P)^{-1 / 3}\right)\right) \gg(\log P)^{-3}(\log \log P)^{3}$.

We use (27), (294), (311), (320), (322), (323), (325), (327), (328) and (334) and we find that if $\alpha=0.00983$ then the estimate (293) holds. It remains to notice that this $\alpha$ satisfies $101<\alpha^{-1}<102$ and the proof of Theorem 1 is complete.

\section{The proof of Theorem 2.}

We recall that in this section bold style letters denote four-dimensional vectors. We also assume that the components of $\mathbf{d}$ are always squarefree. Now we define

$$
\begin{aligned}
S_{\mathbf{d}}(q, m, \mathbf{n}) & =\prod_{i=1}^{4} S\left(q, m d_{i}^{2}, n_{i}\right), \quad S_{\mathbf{d}}(q, m)=S_{\mathbf{d}}(q, m, \mathbf{0}), \\
I_{\mathbf{d}}(\beta, \mathbf{u}) & =\prod_{i=1}^{4} I\left(\beta, u_{i} d_{i}^{-1}\right), \quad f_{\mathbf{d}}(\alpha)=\prod_{i=1}^{4} f_{d_{i}}(\alpha)
\end{aligned}
$$

and let

$$
T_{N}(q, \mathbf{d})=\left((q, N)\left(q, d_{1}^{2}\right) \ldots\left(q, d_{4}^{2}\right)\right)^{1 / 2} .
$$

An important point in [1] is the estimation of the sum

$$
V(q, \mathbf{d}, \mathbf{n}, v, N)=\sum_{a(q)^{*}} e_{q}(v \bar{a}-N a) S_{\mathbf{d}}(q, a, \mathbf{n}) .
$$


In Lemma 1 of [1] Brüdern and Fouvry use estimates for Kloosterman and Salié sums and prove the inequality

$$
V(q, \mathbf{d}, \mathbf{n}, v, N) \ll q^{5 / 2+\varepsilon} T_{N}(q, \mathbf{d}) .
$$

From (338) follows, in particular, that the singular series

$$
\Sigma_{1}(\mathbf{d}, N)=\sum_{q=1}^{\infty} q^{-4} V(q, \mathbf{d}, \mathbf{0}, 0, N)
$$

is absolutely convergent. The arithmetic properties of the function $\Sigma_{1}(\mathbf{d}, N)$ are studied in detail in Section 2.4 of [1]. Define

$$
B(\mathbf{d}, N)=\sum_{\substack{x_{1}^{2}+x_{2}^{2}+x_{3}^{2}+x_{4}^{2}=N \\ x_{i}=0\left(d_{i}\right)}} \omega\left(x_{1}\right) \ldots \omega\left(x_{4}\right)
$$

and

$$
R(\mathbf{d}, N)=B(\mathbf{d}, N)-\kappa_{1} P^{2} \frac{\Sigma_{1}(\mathbf{d}, N)}{d_{1} d_{2} d_{3} d_{4}},
$$

where $\omega, \kappa_{1}$ and $\Sigma_{1}$ are specified by (18), (24) and (339), respectively. For any positive $D$ we define

$$
\mathcal{H}^{*}(D)=\sum_{\left(\mathcal{D}^{*}\right)}|R(\mathbf{d}, N)|,
$$

where $\sum_{\left(\mathcal{D}^{*}\right)}$ means that the summation is taken over squarefree odd integers $d_{1}, d_{2}, d_{3}, d_{4} \leq D$. We prove the following

Proposition 3. Suppose that $D \leq P^{1 / 8-10 \varepsilon}$. Then we have

$$
\mathcal{H}^{*}(D) \ll P^{2-\varepsilon} .
$$

This statement is an analogue of Theorem 3 from [1]. In this paper Brüdern and Fouvry use the approach of Estermann [4] and their upper bound for $d_{i}$ is $P^{\theta}$, where $\theta<1 / 11$. Here we use weighted exponential sums and apply Lemma 12 and our result becomes stronger.

Applying the arguments from Section 3 of [1] we can see that our Theorem 2 is a consequence of Proposition 3.

Proof of Proposition 3. It is enough to establish the inequality

$$
\mathcal{H}^{*}(D) \ll P^{3 / 2+10 \varepsilon} D^{4} .
$$

We use again the Kloosterman method. It is clear that

$$
\begin{aligned}
B(\mathbf{d}, N) & =\int_{0}^{1} e(-N \alpha) f_{\mathbf{d}}(\alpha) d \alpha \\
& =\sum_{q \leq P} \sum_{a(q)^{*}} \int_{\mathcal{B}(q, a)} e\left(-N\left(\frac{a}{q}+\beta\right)\right) f_{\mathbf{d}}\left(\frac{a}{q}+\beta\right) d \beta
\end{aligned}
$$


where the set of integration $\mathcal{B}(q, a)$ and the function $f_{\mathbf{d}}$ are defined by (54) and (336), respectively. We use (335), (336) and Lemma 12 to represent the integrand from the formula above in the form

$$
\frac{P^{4} e\left(-N\left(\frac{a}{q}+\beta\right)\right)}{q^{4} d_{1} d_{2} d_{3} d_{4}} \sum_{\substack{\mathbf{n} \in \mathbb{Z}^{4} \\\left|n_{i}\right| \leq d_{i} P^{\varepsilon}}} S_{\mathbf{d}}(q, a, \mathbf{n}) I_{\mathbf{d}}\left(\beta N,-P q^{-1} \mathbf{n}\right)+\mathcal{O}\left(P^{-A}\right) .
$$

We substitute this expression for the integrand in (343) and change the variable of integration to get

$$
\begin{array}{r}
B(\mathbf{d}, N)=\frac{P^{2}}{d_{1} d_{2} d_{3} d_{4}} \sum_{q \leq P} q^{-4} \sum_{\substack{\mathbf{n} \in \mathbb{Z}^{4} \\
\left|n_{i}\right| \leq d_{i} P^{\varepsilon}}} \sum_{a(q)^{*}} \int_{\mathcal{B}^{\prime}(q, a)} e_{q}(-N a) e(-\beta) \\
\times S_{\mathbf{d}}(q, a, \mathbf{n}) I_{\mathbf{d}}\left(\beta,-P q^{-1} \mathbf{n}\right) d \beta+\mathcal{O}\left(P^{-A}\right),
\end{array}
$$

where the set $\mathcal{B}^{\prime}(q, a)$ is defined by (62). Then we change the order of integration and summation over $a$ to find that

$$
\begin{aligned}
& B(\mathbf{d}, N)=\frac{P^{2}}{d_{1} d_{2} d_{3} d_{4}} \sum_{q \leq P} q^{-4} \sum_{\substack{\mathbf{n} \in \mathbb{Z}^{4} \\
\left|n_{i}\right| \leq d_{i} P^{\varepsilon}}} \int_{|\beta| \leq \frac{P}{q}} e(-\beta) I_{\mathbf{d}}\left(\beta,-P q^{-1} \mathbf{n}\right) \\
& \times \sum_{\substack{a(q)^{*} \\
\bar{a} \in \mathcal{A}(q, \beta)}} e_{q}(-N a) S_{\mathbf{d}}(q, a, \mathbf{n}) d \beta+\mathcal{O}\left(P^{-A}\right),
\end{aligned}
$$

where $\mathcal{A}(q, \beta)$ is the set of residue classes modulo $q$, whose properties were described in Section 3.4.2. In particular, there exists a function $\sigma(v, q, \beta)$, satisfying (99) and such that

$$
\sum_{\substack{a(q)^{*} \\ \bar{a} \in \mathcal{A}(q, \beta)}} \cdots=\sum_{-q / 2<v \leq q / 2} \sigma(v, q, \beta) \sum_{a(q)^{*}} e_{q}(\bar{a} v) \ldots
$$

We represent $B(\mathbf{d}, N)$ in the form

$$
B(\mathbf{d}, N)=B_{1}+B_{2}+\mathcal{O}\left(P^{-A}\right),
$$

where in $B_{1}$ the integration is taken over $\beta$ such that $|\beta| \leq P(2 q)^{-1}$, whilst in $B_{2}$ we integrate over $\beta$ satisfying $P(2 q)^{-1}<|\beta| \leq P q^{-1}$. 
Consider $B_{2}$. From (99), (337), (344) and (345) we obtain

$$
\begin{aligned}
B_{2}=\frac{P^{2}}{d_{1} d_{2} d_{3} d_{4}} \sum_{q \leq P} q^{-4} \sum_{\substack{\mathbf{n} \in \mathbb{Z}^{4} \\
\left|n_{i}\right| \leq d_{i} P^{\varepsilon}}} \int_{\frac{P}{2 q} \leq|\beta| \leq \frac{P}{q}} I_{\mathbf{d}}\left(\beta,-P q^{-1} \mathbf{n}\right) \\
\quad \times e(-\beta) \sum_{-q / 2<v \leq q / 2} \sigma(v, q, \beta) V(q, \mathbf{d}, \mathbf{n}, v, N) d \beta \\
\ll \frac{P^{2}}{d_{1} d_{2} d_{3} d_{4}} \sum_{q \leq P} q^{-4} \sum_{\substack{\mathbf{n} \in \mathbb{Z}^{4} \\
\left|n_{i}\right| \leq d_{i} P^{\varepsilon}}} \sum_{|v| \leq P}(1+|v|)^{-1} \\
\times|V(q, \mathbf{d}, \mathbf{n}, v, N)| \int_{\frac{P}{2 q}}^{\infty}\left|I_{\mathbf{d}}\left(\beta,-P q^{-1} \mathbf{n}\right)\right| d \beta .
\end{aligned}
$$

Applying (336) and Lemma 9 (iii) we find that

$$
\int_{\frac{P}{2 q}}^{\infty}\left|I_{\mathbf{d}}\left(\beta,-P q^{-1} \mathbf{n}\right)\right| d \beta \ll \int_{\frac{P}{2 q}}^{\infty} \beta^{-2} d \beta \ll q P^{-1}
$$

and from (338), (347) and (348) we obtain

$$
B_{2} \ll P^{1+6 \varepsilon} \sum_{q \leq P} q^{-1 / 2} T_{N}(q, \mathbf{d}) .
$$

Consider now $B_{1}$. In this case the set $\mathcal{A}(q, \beta)$ is a complete system of residues modulo $q$ and we have

$$
B_{1}=\frac{P^{2}}{d_{1} d_{2} d_{3} d_{4}} \sum_{q \leq P} q^{-4} \sum_{\substack{\mathbf{n} \in \mathbb{Z}^{4} \\\left|n_{i}\right| \leq d_{i} P^{\varepsilon}}} J(q, \mathbf{d}, \mathbf{n}, N) V(q, \mathbf{d}, \mathbf{n}, 0, N)
$$

where

$$
J(q, \mathbf{d}, \mathbf{n}, N)=\int_{|\beta| \leq \frac{P}{2 q}} e(-\beta) I_{\mathbf{d}}\left(\beta,-P q^{-1} \mathbf{n}\right) d \beta .
$$

Using (24), (336), (348) and Lemma 10 (ii) we find that

$$
J(q, \mathbf{d}, \mathbf{n}, N)= \begin{cases}\kappa_{1}+\mathcal{O}\left(q P^{-1}\right) & \text { if } \mathbf{n}=\mathbf{0}, \\ \mathcal{O}\left(q P^{-1+2 \varepsilon}\left(\sum_{i=1}^{4}\left|n_{i}\right| d_{i}^{-1}\right)^{-1}\right) & \text { otherwise } .\end{cases}
$$

From (338), (339), (350) and (351) we obtain

$$
B_{1}=\kappa_{1} P^{2} \frac{\Sigma_{1}(\mathbf{d}, N)}{d_{1} d_{2} d_{3} d_{4}}+\mathcal{O}\left(B_{3}\right)+\mathcal{O}\left(B_{4}\right)+\mathcal{O}\left(B_{5}\right),
$$


where

$$
\begin{aligned}
B_{3} & =\frac{P^{2}}{d_{1} d_{2} d_{3} d_{4}} \sum_{q>P} q^{-3 / 2+\varepsilon} T_{N}(q, \mathbf{d}), \\
B_{4} & =\frac{P^{1+3 \varepsilon}}{d_{1} d_{2} d_{3} d_{4}} \sum_{q \leq P} q^{-1 / 2} T_{N}(q, \mathbf{d}), \\
B_{5} & =\frac{P^{1+3 \varepsilon}}{d_{1} d_{2} d_{3} d_{4}} \sum_{q \leq P} q^{-1 / 2} T_{N}(q, \mathbf{d}) \sum_{\substack{\mathbf{0} \neq \mathbf{n} \in \mathbb{Z}^{4} \\
\left|n_{i}\right| \leq d_{i} P^{\varepsilon}}}\left(\sum_{i=1}^{4}\left|n_{i}\right| d_{i}^{-1}\right)^{-1} .
\end{aligned}
$$

Using the last three formulas as well as (349) we can establish that

$$
\sum_{\left(\mathcal{D}^{*}\right)}\left|B_{i}\right| \ll P^{3 / 2+10 \varepsilon} D^{4} \quad \text { for } \quad i=2,3,4,5 .
$$

The calculations are not difficult and we leave them to the reader.

We take into account (340), (341), (346), (352) and (353) and prove that the estimate (342) is correct. This completes the proof of Proposition 3. 


\section{References}

[1] J. Brüdern , E. Fouvry, Lagrange's Four Squares Theorem with almost prime variables, J. Reine Angew. Math. 454 (1994), 59-96.

[2] J. Brüdern , E. Fouvry, Le crible à vecteurs, Compos. Math., 102 (1996), $337-355$.

[3] H. Davenport, Multiplicative Number Theory, Third ed., Springer, 2000, (revised by H.L. Montgomery).

[4] T. Estermann, A new application of the Hardy-Littlewood-Kloosterman method, Proc. London Math. Soc., 12 (1962), 425-444.

[5] T. Estermann, On Kloosterman's sum, Mathematika, 8, (1961), 83-86.

[6] G. Greaves, On the representation of a number in the form $x^{2}+y^{2}+p^{2}+q^{2}$ where $p$ and $q$ are odd primes, Acta Arith. 29 (1976), 257-274.

[7] G. H. Hardy, E. M. Wright, An introduction to the theory of numbers, Fifth ed., Oxford Univ. Press, 1979.

[8] D.R. Heath-Brown, The square sieve and consecutive square-free numbers, Math. Ann. 226 (1984), 251-259.

[9] D.R. Heath-Brown, Cubic forms in ten variables, Proc. London Math. Soc. 47 (1983), 225-257.

[10] L.K. Hua, Some results in the additive prime number theory, Quart. J. Math. Oxford, 9 (1938), 68-80.

[11] L.K. Hua, Introduction to number theory, Springer, 1982.

[12] H. Iwaniec, On sums of two norms from cubic fields, Journées de théorie additive des nombres, Université de Bordeaux I, (1977), 71-89.

[13] H. Iwaniec, Rosser's sieve, Acta Arith. 36 (1980), 171-202.

[14] H. Iwaniec, A new form of the error term in the linear sieve, Acta Arith. 37 (1980), 307-320.

[15] A.A. Karatsuba, Basic analytic number theory, Springer, 1993.

[16] H.D. Kloosterman, On the representation of numbers in the form $a x^{2}+$ $b y^{2}+c z^{2}+d t^{2}$, Acta Math. 49 (1926), 407-464.

[17] V.A. Plaksin, An asymptotic formula for the number of solutions of a nonlinear equation for prime numbers, Math. USSR Izv. 18 (1982), 275-348.

[18] P. Shields, Some applications of the sieve methods in number theory, Thesis, University of Wales 1979. 
[19] D.I. Tolev, Arithmetic progressions of prime-almost-prime twins, Acta Arith. 88 (1999), 67-98.

[20] D.I. Tolev, Additive problems with prime numbers of special type, Acta Arith. 96 (2000), 53-88.

[21] A. Weil, On some exponential sums, Proc. Nat. Acad. Sci. U.S.A., 34, (1948), 204-207.

Mathematical Institute

$24-29$ St. Giles'

Oxford OX1 3LB

England

Email: rhb@maths.ox.ac.uk

Department of Mathematics

Plovdiv University "P. Hilendarski"

24 "Tsar Asen" str.

Plovdiv 4000

Bulgaria

Email: dtolev@pu.acad.bg 